\title{
Biomarker-based Dose-finding Designs for Single- or Multiple-Agent Phase I Trials
}

\author{
Yuan Xue \\ Tianjin, P.R.China \\ B.S., Peking University, P.R.China, 2010 \\ M.S., University of Virginia, United States, 2012 \\ A Dissertation Presented to the Graduate Faculty \\ of the University of Virginia in Candidacy for the Degree of \\ Doctor of Philosophy
}

Department of Statistics

University of Virginia

May, 2014 


\begin{abstract}
The primary goal of a Phase I clinical trial is to estimate the maximum tolerated dose (MTD). The MTD is defined as the highest dose which can be administered with a "tolerable" level of toxicity. The "tolerable" level of toxicity is based on the probability that a patient in the trial experiences a dose-limiting toxicity (DLT). This dissertation addresses two practical considerations: heterogeneous toxicity response and a partial ordering for the probability of toxicity for available treatments.

First, the majority of methods for the Phase I trials are designed for a homogeneous toxicity response, resulting in a unique MTD for the broad patient population. However, patients may naturally differ in the way they react to a treatment. Clinical useful biomarkers which affect the probability of a DLT have been developed as more toxicity biomarker studies have been done. We propose a new design which chooses a distinct MTD for individual patient by use of toxicity biomarker information, thus contributing to a proper and better treatment for individual patient.

Second, for multiple-agent trials we may be able to identify the order of the probability of toxicity for only a subset of the available treatments, which is a "partial order", in contrast to single-agent trial problems whose order of the probability of toxicity for all the treatments is fully known, which is a "simple order". We propose a biomarker-based design for which the ordering is not fully known.

The operating characteristics of the proposed designs for simple order and partial order are investigated through extensive simulation studies. A discuss of the theoretical properties is provided. We also employ implementing model selection techniques, specifically BIC model selection method to improve the performance of the biomarker-based designs. We close with some conclusions drawn from the proposed biomarker-based designs, as well as some topics for further research.
\end{abstract}




\section{Acknowledgements}

I would like to express my sincere gratitude to my advisors: Professor Mark Conaway and Professor Feifang Hu. This dissertation could not have been written without their guidance. Prof. Conaway provided me a great number of creative ideas for my research and guided me how to move on. He was always there when I was stuck by tough problems in the research. He always identified the problems in the shortest time and suggested the possible directions I could work on, which was especially needed for me as a beginner for research. His great idea about this research area will benefit my research in the future. Prof. Hu taught me five courses. My understanding of this research field is accumulated from the courses I took from him, our meetings and discussions. He not only gave me academic guidance but also life advices. I have learned a lot from his positive attitude towards life, which will benefit me for a lifetime. He provided me with various opportunities, which have become the cornerstones to build my career. I feel very honored and fortunate to be a student of Prof. Conaway and Prof. Hu. I consider my decision of choosing them as my advisors as one of the most important life-changing decisions I have ever made.

I would also like to thank my other committee members: Professor Jianhui Zhou and Professor Nolan Wages, for reading my dissertation and giving useful comments. Prof. Wages, with his rich experience in design of Phase I trials, provided many constructive ideas for my research. Thanks must also go to all the faculty and staff in the Department of Statistics, who have created a supportive academic atmosphere. In particular, I am grateful to Christopher Tait for sharing his option and materials about research in design of Phase I trials. I treasure the memories I made with my dear classmates: Yanqing Hu, Zhenjun Ma, Yue Liu, Wei Ma, Xiaoxiao Tang, 
Feiyang Niu, Jingwei Wu, Christopher Tait, Robert Pfister, Caitlin Steiner, Maria Ferrara, Xiaoming Li, Chenyi Pan, etc.

Finally, I would like to thank my parents and my grandparents for their continued long-distance support. They are my motivation to keep fighting. I would like to thank all people who have helped me during my Ph.D. study. 


\section{Contents}

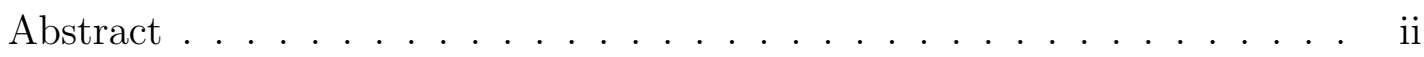

Acknowledgements . . . . . . . . . . . . . . . . iii

1 Introduction 1

1.1 Background . . . . . . . . . . . . . . . . . . . . 1

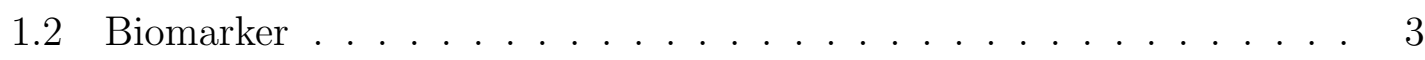

1.2.1 An Example of Categorical Toxicity Biomarker . . . . . . . . 4

1.2.2 An Example of Quantitative Toxicity Biomarker . . . . . . . 5

1.3 Partial Order . . . . . . . . . . . . . . . . . 6

1.4 Literature Review . . . . . . . . . . . . . . . . . . . 11

1.4.1 Up-and-Down Escalation Schemes . . . . . . . . . . . . . . 11

1.4.2 Random Walk Method ... . . . . . . . . . . . 12

1.4.3 Continual Reassessment Method (CRM) . . . . . . . . . 13

1.4.4 Partial Order CRM . . . . . . . . . . . . . . . 16

1.4.5 Two Sample CRM . . . . . . . . . . . . . . . 18

1.5 Outline of Dissertation . . . . . . . . . . . . . . . . . . 19

2 Biomarker-based Design for Single-agent Trial 21

2.1 Statistical Method . . . . . . . . . . . . . . . . 21

2.1.1 Toxicity Probability Model . . . . . . . . . . . . . . . . . 21 
2.1 .2 Inference . . . . . . . . . . . . . . . . . 23

2.2 Numerical Studies . . . . . . . . . . . . . . . . . . . . . 25

2.3 Model Selection between Biomarker Model and Non-biomarker Model 34 2.3.1 Statistical Method . . . . . . . . . . . . . 34

2.3 .2 Numerical Studies . . . . . . . . . . . . . . . . . 36

$3 \quad$ Theoretical Properties $\quad 39$

3.1 The Establish of Maximum Likelihood Estimation . . . . . . . . . . . 46

3.2 Convergence . . . . . . . . . . . . . . . . . 53

3.3 Asymptotic normality . . . . . . . . . . . . . . . . . . 59

4 Biomarker-based Design for Multiple-agent Trial 63

4.1 Statistical Method . . . . . . . . . . . . . . . 63

4.1.1 Toxicity Probability Model . . . . . . . . . . . . . . 63

4.1 .2 Inference . . . . . . . . . . . . . . . 65

4.1.3 Additional Randomization for Order Selection . . . . . . . . . 68

4.2 Numerical Studies . . . . . . . . . . . . . . . . . . . . . . . 68

4.3 Model Selection between Biomarker Model and Non-biomarker Model 78

4.3.1 Statistical Method . . . . . . . . . . . . 78

4.3.2 Numerical Studies . . . . . . . . . . . . . . . . . . . . . 81

5 Conclusion and Future Research $\quad 85$

5.1 Conclusion . . . . . . . . . . . . . . . . . 85

5.2 Further Research . . . . . . . . . . . . . . . . . . 87

$\begin{array}{ll}\text { A Appendix } & 90\end{array}$

$\begin{array}{ll}\text { Bibliography } & 109\end{array}$ 


\section{Chapter 1}

\section{Introduction}

\subsection{Background}

The primary goal of a Phase I clinical trial is to estimate the maximum tolerated dose (MTD). The MTD is defined as the highest dose which can be administered with a "tolerable" level of toxicity. The "tolerable" level of toxicity is based upon the probability that a patient in the trial experiences a dose-limiting toxicity (DLT). The DLT refers to the appearance of side effects during treatment which are severe enough to prevent further increase in dosage or strength of treatment agent or to prevent continuation of treatment at any dosage level.

Numerous statistical methods have been proposed for Phase I trials. With few exceptions (e.g., Durham, Flournoy, and Rosenberger, 1997; Babb, Rogatko, and Zacks, 1998), these methods have focused on identifying the MTD from a fixed set of $k$ pre-specified dose levels $d_{1}, d_{2}, \ldots, d_{k}$ in which toxicity is measured as a binary outcome indicating whether a patient experienced a DLT or not.

First, the majority of the statistical methods for the design of Phase I dosefinding studies in oncology are based on the assumption that the toxicity response 
is homogeneous among the broad patient population, further leading to a unique MTD. This assumption is generally a reasonable one, when there is no available clinical biomarker that is informative for toxicity response. However, clinical useful biomarkers which affect the probability of a DLT have been developed. From the point of personalized medicine, which aims to customize treatment by using information about an individual patient, distinct MTDs chosen by use of an informative toxicity biomarker and contributing to proper and better treatment for individual patient would be preferred.

Second, the majority of the statistical methods for the experimental design of Phase I dose-finding studies are based on the assumption of monotonically increasing probability of toxicity as the dose increases. For single-agent trials, the assumption is straightforward. High dose levels are expected to have larger risk of DLT. Under the terminology of Robertson, Wright and Dykstra (1988), a "simple order" is defined that the ordering of the probability of toxicity between any two doses is known and the higher dose corresponds to a larger probability of toxicity. However, not all Phase I trials involve a single cytotoxic agent. The use of multiple-agent treatments is becoming more commonplace in dose-finding studies. Phase I trials involving multiple-agent are more challenging in MTD estimation than single-agent studies. It is reasonable to assume monotonicity for each agent separately, but, when combined, the ordering may not be fully known. We may be able to identify the order of the probability of toxicity for only a subset of the available treatments, resulting in a "partial order". Thus, it may not be clear which dose should be the next escalation dose. Therefore, the use of biomarker under the partial order restriction would be a crucial problem in personalized medicine. 


\subsection{Biomarker}

Improved understanding of biology and advances in biotechnology bring in the concept of personalized medicine. Personalized medicine proposes the customization of treatment, with decisions being tailored to the individual patient by use of genetic or other information. A key component of this concept is the development of biomarkers which can guide treatment application.

Clinical biomarkers that aid in making treatment decision will play an important role in achieving personalized medicine. Although the development of biomarkers bring us closer to the concept of personalized treatment, there are few statistical methods for designing these trials. Traditionally, most designs of clinical trials focus on obtaining an average estimate in a broad patient population. The difficulty in taking advantage of biomarkers is that the trials often require more complex designs. Current statistical methodology about biomarkers mainly focuses on Phase II and Phase III efficacy and effectiveness study. Until now, there are very few methods about biomarkers that have been developed for Phase I dose-finding studies. However, for the purpose of personalized medicine, it is not only necessary to make use of biomarker in efficacy and effectiveness study, but it is also reasonable to choose distinct MTDs for individual patient by use of biomarkers. Patients with high MTD receive treatment with high dose level which contributes to better efficacy and effectiveness; patients with low MTD receive treatment with low dose level which helps avoid side effects.

Biomarkers that are informative for clinical outcome are broadly categorized as predictive and prognostic biomarkers (Freidlin, McShane and Korn 2010). Predictive biomarkers help to assess the most likely response to a particular treatment. For example, in advanced colorectal cancer, the benefit of cetuximab appears to be 
limited to patients with tumors who have the wild-type KRAS genotype (Karapetis, Khambata-Ford, Jonker, et al 2008). Prognostic biomarkers show the progression of disease with or without treatment and have some implications for therapy decisions. Prognostic biomarkers classify patients receiving standard treatment into subgroups with distinct expected clinical outcomes. For example, if a prognostic biomarker can identify a group of patients with very low risk of recurrence, additional treatment might not be considered, whereas patients with higher risk of recurrence would be treated. Note that biomarkers that predict toxicity to a certain agent are often treated as a separate type of biomarker. However, for the purpose of biomarker designs, toxicity biomarkers can be considered as a type of predictive biomarker (Mandrekar, Grothey, Goetz, and Sargent, 2005).

\subsubsection{An Example of Categorical Toxicity Biomarker}

UGT1A1 genotype is an example of categorical toxicity biomarker. Kim et al. (2013) conducted a UGT1A1 *28 and ${ }^{*} 6$ genotype-directed Phase I trial of irinotecan with fixed-dose capecitabine in Korean patients with histological confirmed metastatic colorectal cancer (Table 1.1). The UGT1A1 *6 genotype has been exclusively identified in Asian population (Akaba et al., 1998; Fujita et al., 2007). Fifty patients were genotyped for UGT1A1 $* 28$ and $* 6$, and grouped according to the numbers of defective alleles (DA): 0 (wild type: $*^{*} 1 / *^{*}$ ), 1 (containing only one of the ${ }^{*} 28$ or $* 6$ allele: ${ }^{*} 1 /{ }^{*} 28$ or $\left.*^{*} 1 /{ }^{*} 6\right)$, and $2\left(*^{*} 28 /{ }^{*} 28, *^{*} /{ }^{*} 6\right.$, or double heterozygous for $*^{*} 1 /{ }^{*} 28$ and $\left.*^{*} 1 /{ }^{*} 6\right)$. The MTD of irinotecan was $350 \mathrm{mg} / \mathrm{m}^{2}$ for the 0 and 1 DA groups, and $200 \mathrm{mg} / \mathrm{m}^{2}$ for the 2 DA group.

Sunakawa et al. (2012) conducted a Phase I study of combination chemotherapy with 5-fluorouracil (5-FU), leucovorin, irinotecan and oxaliplatin (FOLFOXIRI) 
Table 1.1: Irinotecan dose level in Phase I trial with fixed-dose capecitabine in patients with metastatic colorectal cancer

\begin{tabular}{ccccccc}
\hline & \multicolumn{6}{c}{ Treatment } \\
\cline { 2 - 7 } Level & $d_{1}$ & $d_{2}$ & $d_{3}$ & $d_{4}$ & $d_{5}$ & $d_{6}$ \\
\hline Irinotecan $\mathrm{mg} / \mathrm{m}^{2}$ & 200 & 240 & 280 & 320 & 350 & 380 \\
\hline
\end{tabular}

in Japanese patients with advanced colorectal cancer who harbor UGT1A1 * $1 /{ }^{*} 1$, ${ }^{*} 1 /{ }^{*} 6$ or $*^{*} 1 / *^{*} 28$. Patients with UGT1A $1 *^{*} 6 / *_{6},{ }^{*} 28 / *^{*} 28$ and $*^{*} 6 / *^{*} 28$ genotypes were excluded, because these UGT1A1 genotypes are linked to severe irinotecan-induced toxicity. A total of ten patients were studied. The MTD of FOLFOXIRI in these patients was $165 \mathrm{mg} / \mathrm{m}^{2}$ irinotecan, $85 \mathrm{mg} / \mathrm{m}^{2}$ oxaliplatin and 2,400 $\mathrm{mg} / \mathrm{m}^{2}$ 5-FU.

Table 1.2: Treatments used in Phase I trial of combination chemotherapy with 5fluorouracil (5-FU), leucovorin, irinotecan and oxaliplatin (FOLFOXIRI) in patients with advanced colorectal cancer

\begin{tabular}{ccccccc}
\hline & \multicolumn{7}{c}{ Treatment } \\
\cline { 2 - 7 } Treatment & $d_{1}$ & $d_{2}$ & $d_{3}$ & $d_{4}$ & $d_{5}$ & $d_{6}$ \\
\hline Irinotecan $\mathrm{mg} / \mathrm{m}^{2}$ & 120 & 150 & 165 & 150 & 165 & 180 \\
Oxaliplatin $\mathrm{mg} / \mathrm{m}^{2}$ & 85 & 85 & 85 & 85 & 85 & 85 \\
5-FU $\mathrm{mg} / \mathrm{m}^{2}$ & 2,400 & 2,400 & 2,400 & 3,200 & 3,200 & 3,200 \\
\hline
\end{tabular}

\subsubsection{An Example of Quantitative Toxicity Biomarker}

An example of quantitative toxicity biomarker is telomere length (TL). Garg et al. (2012) conducted a comprehensive survey of clinically measurable variables to determine predictors of 5-fluorouracil (5-FU) toxicity. Seventy-three patients with colorectal cancer (CRC) received adjuvant 5-fluorouracil (5FU) / leucovorin (LV) using either the Mayo Clinic or a weekly schedule and were evaluated for clinical toxicity. For the Mayo Clinic schedule 42 patients were given $425 \mathrm{mg} / \mathrm{m}^{2}$ 5-FU 
and $20 \mathrm{mg} / \mathrm{m}^{2} \mathrm{LV}$ daily for 5 days every 4 weeks, while for the weekly schedule 31 patients were given $500 \mathrm{mg} / \mathrm{m}^{2} 5$-FU and $20 \mathrm{mg} / \mathrm{m}^{2} \mathrm{LV}$ weekly for 4 weeks. For the Mayo schedule, the primary toxicities were neutropenia (69\%), mucositis (58\%) and leukopenia $(46 \%)$, with $70 \%$ of patients presenting with haematological toxicity $\geq$ grade I (neutropenia and/or leukopenia), while the primary toxicity for the weekly schedule was diarrhoea (16\%). The study showed that short telomere length of peripheral blood mononuclear cell (PBMNC) and an increased platelet lymphocyte ratio (PLR) were strong predictors of mucositis and haematological toxicity in CRC patients undergoing 5-FU treatment in the adjuvant setting.

Falandry et al. (2012) conducted a multicenter GINECO trial in elderly AOC patients receiving carboplatin. Telomere length (TL) in base pairs was estimated for 109/111 patients. With a cutoff of 5770 base pairs, TL discriminated two groups with significantly different treatment completion rates. Short telomere length patients were at higher risk of severe adverse events and tended to have more unplanned hospital admissions. This study identified TL as a predictive factor of decreased treatment completion, severe adverse events risk and unplanned hospital admissions.

\subsection{Partial Order}

In single-agent trials, "treatment" is synonymous with "dose level". In multipleagent trials, we use "treatment" to denote combinations of agents. For single-agent trials, the ordering of the probability of toxicity is assumed monotonic with dose. For multiple-agent trials, monotonicity is assumed for each agent separately, but the combined ordering may not be fully known, resulting in a "partial order".

Example 1. Patnaik et al. (2000) showed a Phase I study of Paclitaxel and Carbo- 
platin combination for patients with solid tumors. Table 1.3 displays the treatments used in the trial.

Table 1.3: Treatments used in Phase I trial of Paclitaxel and Carboplatin in patients with solid tumor

\begin{tabular}{ccccccc}
\hline & \multicolumn{7}{c}{ Treatment } \\
\cline { 2 - 7 } Agent & $d_{1}$ & $d_{2}$ & $d_{3}$ & $d_{4}$ & $d_{5}$ & $d_{6}$ \\
\hline Paclitaxel & 54 & 67.5 & 81 & 94.5 & 67.5 & 67.5 \\
Carboplatin & 6 & 6 & 6 & 6 & 7.5 & 9 \\
\hline
\end{tabular}

In this example, the ordering of the probability of toxicity for some treatment combinations is unknown. The six dose combinations are denoted as $d_{1}, d_{2}, \ldots, d_{6}$. The toxicity ordering between treatments $d_{1}$ and $d_{2}$ is known because the level of Carboplatin remains the same while the level of Paclitaxel increases. However, the ordering relationship between $d_{3}$ and $d_{6}$ is unknown because the level of Paclitaxel increases while the level of Carboplatin decreases. Hwang and Peddada (1994) proposed a method for estimating parameters under partial order restriction. The method distinguishes between nodal and nonnodal parameters. The ordering of a nodal parameter is fully known with all other parameters. In simple order, all parameters are nodal. In the partial order example of Table 1.3, parameters 1 and 2 are nodal. $R\left(d_{1}\right)$ is a nodal parameter because $R\left(d_{1}\right) \leq R\left(d_{i}\right)$ for $i \geq 2$. $R\left(d_{2}\right)$ is a nodal parameter because $R\left(d_{2}\right) \geq R\left(d_{1}\right)$ and $R\left(d_{2}\right) \leq R\left(d_{i}\right)$ for $i \geq 3$. If we continue to access the known and unknown toxicity ordering relationships, the following two order relationships could be determined: $d_{1} \rightarrow d_{2} \rightarrow d_{3} \rightarrow d_{4}$ and $d_{1} \rightarrow d_{2} \rightarrow d_{5} \rightarrow d_{6}$. In these diagrams, treatments whose ordering are known are connected by lines, with parameters on the right larger than parameters on the left. 
Figure 1.1: Treatments used in Phase I trial of Paclitaxel and Carboplatin in patients with solid tumor

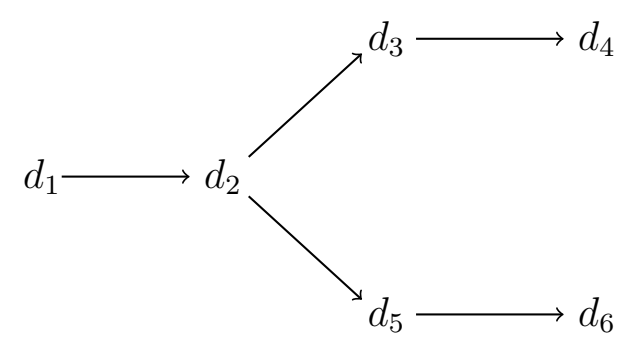

Escalation to a previously untried dose depends on a specification prior to the trial of "possible escalation treatments" associated with each treatment. For example, the possible escalation treatments for $d_{2}$ are $d_{3}$ and $d_{4}$. Taking into account the subset of dose combinations for which we know the toxicity order, we can formulate all the possible $M$ orderings of the probability of toxicity. In this example, the trial requires the investigation of the following six simple orders:

$$
\begin{aligned}
& M_{1}: d_{1} \rightarrow d_{2} \rightarrow d_{3} \rightarrow d_{4} \rightarrow d_{5} \rightarrow d_{6} \\
& M_{2}: d_{1} \rightarrow d_{2} \rightarrow d_{3} \rightarrow d_{5} \rightarrow d_{4} \rightarrow d_{6} \\
& M_{3}: d_{1} \rightarrow d_{2} \rightarrow d_{3} \rightarrow d_{5} \rightarrow d_{6} \rightarrow d_{4} \\
& M_{4}: d_{1} \rightarrow d_{2} \rightarrow d_{5} \rightarrow d_{6} \rightarrow d_{3} \rightarrow d_{4} \\
& M_{5}: d_{1} \rightarrow d_{2} \rightarrow d_{5} \rightarrow d_{3} \rightarrow d_{6} \rightarrow d_{4} \\
& M_{6}: d_{1} \rightarrow d_{2} \rightarrow d_{5} \rightarrow d_{3} \rightarrow d_{4} \rightarrow d_{6}
\end{aligned}
$$

Example 2. Berenson et al. (2009) described a Phase I study of Samarium lexidronam and Bortezomib combination for treatment of relapsed or refractory multiple myeloma. The DLT was defined by a patient experiencing a grade $3+$ neutrophenia. Table 1.4 shows the treatments. 
Table 1.4: Treatments used in Phase I trial of Samarium lexidronam and Bortezomib

\begin{tabular}{ccccccc}
\hline & \multicolumn{7}{c}{ Treatment } \\
\cline { 2 - 7 } Agent & $d_{1}$ & $d_{2}$ & $d_{3}$ & $d_{4}$ & $d_{5}$ & $d_{6}$ \\
\hline $\mathrm{Sm} \mathrm{mCi} / \mathrm{kg}$ & 0.25 & 0.5 & 1.0 & 0.25 & 0.5 & 1.0 \\
Bortezomib $\mathrm{mg} / \mathrm{m}^{2}$ & 1.0 & 1.0 & 1.0 & 1.3 & 1.3 & 1.3 \\
\hline
\end{tabular}

In these diagrams, combinations whose orderings are known are connected by arrows.

Figure 1.2: Treatments used in Phase I trial of Samarium lexidronam and Bortezomib

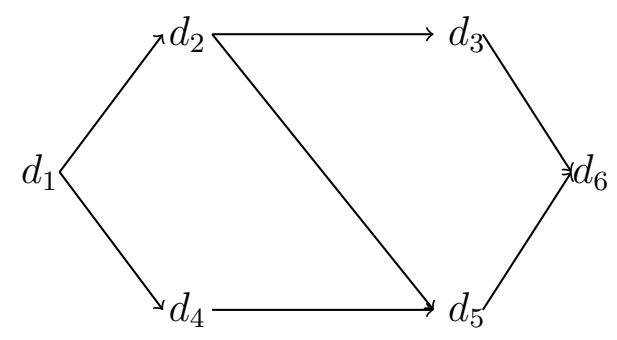

In this example, there are five possible orderings of probability of toxicity:

$$
\begin{aligned}
& M_{1}: d_{1} \rightarrow d_{2} \rightarrow d_{3} \rightarrow d_{4} \rightarrow d_{5} \rightarrow d_{6} \\
& M_{2}: d_{1} \rightarrow d_{2} \rightarrow d_{4} \rightarrow d_{3} \rightarrow d_{5} \rightarrow d_{6} \\
& M_{3}: d_{1} \rightarrow d_{2} \rightarrow d_{4} \rightarrow d_{5} \rightarrow d_{3} \rightarrow d_{6} \\
& M_{4}: d_{1} \rightarrow d_{4} \rightarrow d_{2} \rightarrow d_{3} \rightarrow d_{5} \rightarrow d_{6} \\
& M_{5}: d_{1} \rightarrow d_{4} \rightarrow d_{2} \rightarrow d_{5} \rightarrow d_{3} \rightarrow d_{6}
\end{aligned}
$$

EXAMPLE 3. Lokich (2001) described a dose-finding study of combinations of Topotecan and Irinotecean. Table 1.5 displays the treatments. 
Table 1.5: Treatments used in Phase I trial of Irinotecean and Topotecan

\begin{tabular}{ccccccccc}
\hline & \multicolumn{8}{c}{ Treatment } \\
\cline { 2 - 8 } Agent & $d_{1}$ & $d_{2}$ & $d_{3}$ & $d_{4}$ & $d_{5}$ & $d_{6}$ & $d_{7}$ & $d_{8}$ \\
\hline Irinotecean $\mathrm{mg} / \mathrm{m}^{2}$ & 50 & 50 & 75 & 75 & 100 & 100 & 125 & 125 \\
Topotecan $\mathrm{mg} / \mathrm{m}^{2}$ & 1.0 & 1.5 & 1.0 & 1.5 & 1.0 & 1.5 & 1.0 & 1.5 \\
\hline
\end{tabular}

In these diagrams, combinations whose orderings are known are connected by arrows.

Figure 1.3: Treatments used in Phase I trial of Irinotecean and Topotecan

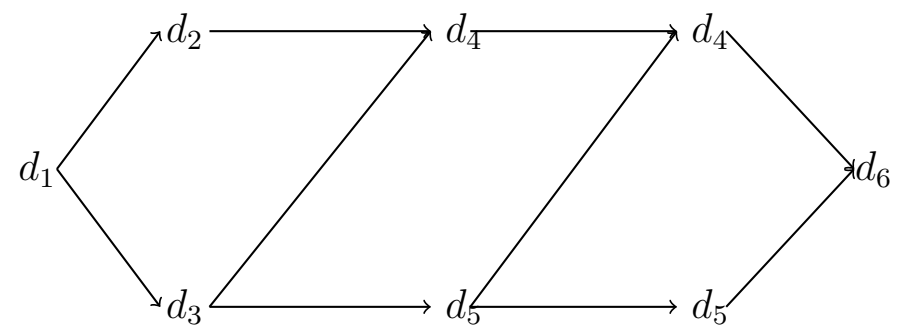

In this example, there are eight possible orderings of probability of toxicity:

$$
\begin{aligned}
& M_{1}: d_{1} \rightarrow d_{2} \rightarrow d_{3} \rightarrow d_{4} \rightarrow d_{5} \rightarrow d_{6} \rightarrow d_{7} \rightarrow d_{8} \\
& M_{2}: d_{1} \rightarrow d_{3} \rightarrow d_{2} \rightarrow d_{4} \rightarrow d_{5} \rightarrow d_{6} \rightarrow d_{7} \rightarrow d_{8} \\
& M_{3}: d_{1} \rightarrow d_{2} \rightarrow d_{3} \rightarrow d_{5} \rightarrow d_{4} \rightarrow d_{6} \rightarrow d_{7} \rightarrow d_{8} \\
& M_{4}: d_{1} \rightarrow d_{2} \rightarrow d_{3} \rightarrow d_{4} \rightarrow d_{5} \rightarrow d_{7} \rightarrow d_{6} \rightarrow d_{8} \\
& M_{5}: d_{1} \rightarrow d_{3} \rightarrow d_{2} \rightarrow d_{5} \rightarrow d_{4} \rightarrow d_{6} \rightarrow d_{7} \rightarrow d_{8} \\
& M_{6}: d_{1} \rightarrow d_{3} \rightarrow d_{2} \rightarrow d_{4} \rightarrow d_{5} \rightarrow d_{7} \rightarrow d_{6} \rightarrow d_{8} \\
& M_{7}: d_{1} \rightarrow d_{2} \rightarrow d_{3} \rightarrow d_{5} \rightarrow d_{4} \rightarrow d_{7} \rightarrow d_{6} \rightarrow d_{8}
\end{aligned}
$$


$M_{8}: d_{1} \rightarrow d_{3} \rightarrow d_{2} \rightarrow d_{5} \rightarrow d_{4} \rightarrow d_{7} \rightarrow d_{6} \rightarrow d_{8}$

\subsection{Literature Review}

\subsubsection{Up-and-Down Escalation Schemes}

The most traditional dose-finding design is the Up-and-Down escalation schemes. It is a simple, algorithm-based approach. Storer (1989) reviewed the Up-and-Down escalation schemes. Four single-stage designs were described in the paper:

Design A (traditional " $3+3$ " method) Groups of three patients are treated. Escalation occurs if no toxicity is observed in all three; otherwise, an additional three patients are treated at the same dose level. If only one of six has toxicity, escalation again continues; otherwise, the trial stops and the lower dose is considered to be the MTD.

Design B Single patient is treated. The next patient is treated at the next lower dose level if a toxic response is observed, otherwise at the next higher dose level.

Design C Similar to design B, except that two consecutive nontoxic responses must be obtained before escalation occurs, whereas de-escalation occurs whenever a toxic response is seen.

Design D Groups of three patients are treated. Escalation occurs if no toxicity is seen and de-escalation if more than one patient has toxicity. If a single patient has toxicity, the next group of three is treated at the same dose level.

Storer (1989) also proposed some two-stage designs in the paper: BC and BD, where the first stage follows design B until the first toxic response occurs, then the second stage follows. At the end of the trial, use logistic regression to estimate the MTD. The traditional Up-and-Down escalation schemes are not the most effi- 
cient method. They treat many patients with sub-optimal doses and may provide a poor estimate of MTD. However, they are widely accepted, especially the traditional " $3+3$ " method is still widely used in practice because of the advantage of its algorithm-based simplicity.

\subsubsection{Random Walk Method}

Durham et al. (1997) described a random walk rule for the sequential allocation of dose levels to patients in a Phase I clinical trial or a dose-response study. Let $x_{1}, x_{2}, \ldots, x_{k}$ be an ordered set of doses with monotonic toxicity curve. If patient $j$ has just been assigned to dose level $x_{i}$, assign patient $j+1$ with the following rule:

If patient $j$ experienced DLT, assign patient $j+1$ to $x_{i-1}$.

If patient $j$ did not experience DLT, flip a biased coin with probability of heads $b \in[0,0.5]$. The choice of $b$ is related to the target probability of DLT. If it lands head up, assign patient $j+1$ to $x_{i+1}$. If it lands head down, assign patient $j+1$ to $x_{i}$.

With imposed boundary $x_{1}$ and $x_{k}$, simply assign the patient to $x_{1}$ or $x_{k}$, if a potential de-escalation lower than $x_{1}$ or a potential escalation higher than $x_{k}$ occurs.

The estimate of MTD is

$$
X(n)=\frac{\sum_{i=1}^{k} x_{i} N_{i}(n)}{n},
$$

where $n$ is the number of enrolled patients and $N_{i}(n)$ is the number of patients treated at dose $i$. 


\subsubsection{Continual Reassessment Method (CRM)}

O'Quigley, Pepe and Fisher (1990) introduced the continual reassessment method $(\mathrm{CRM})$ as an alternative method for the traditional Up-and Down escalation schemes reviewed by Storer (1989). The original form of CRM is a Bayesian method. CRM sequentially updates the dose-toxicity information to estimate the dose to treat the next patient. Suppose that we have a discrete set of $k$ pre-specified dose levels $d_{1}, d_{2}, \ldots, d_{k}$. CRM models $R(x)$, which is the true probability of toxicity at $X_{j}=$ $x_{j} \in\left\{d_{1}, \ldots, d_{k}\right\}$ via

$$
R\left(x_{j}\right)=\operatorname{Pr}\left(Y_{j}=1 \mid X_{j}=x_{j}\right)=E\left(Y_{j} \mid x_{j}, z_{j}\right)=\psi\left(x_{j}, a\right)
$$

for a simplified functional dose-toxicity curve $\psi\left(d_{i}, a\right)$ and $a$ defined on the set $\mathcal{A}$. The restrictions on $\psi\left(x_{j}, a\right)$ are discussed in O'Quigley, Pepe and Fisher (1990). First, for each $a, \psi(\cdot, a)$ is strictly increasing. Second, $\psi\left(d_{i}, \cdot\right)$ is continuous and strictly monotone in $a$ in the same direction for all $d$. The most commonly used working models are a power model or a logistic model:

1. Power model $\psi\left(d_{i}, a\right)=\alpha_{i}^{a}$, where $\alpha_{i}$ are pre-specified values for each dose level $d_{i}, \alpha_{i}$ are called the skeleton of the working model and satisfy $0<\alpha_{1}<$ $\alpha_{2}<\cdots<\alpha_{k}<1$.

2. Logistic model $\psi\left(d_{i}, a\right)=\frac{\exp \left(a+\alpha_{i}\right)}{1+\exp \left(a+\alpha_{i}\right)}$, where $\alpha_{i}$ are pre-specified values for each dose level $d_{i}$ and satisfy $\alpha_{1}<\alpha_{2}<\cdots<\alpha_{k}$.

After having $j$ patients enrolled, we have data in the form of $\Omega_{j}=\left\{x_{1}, y_{1}, \ldots, x_{j}, y_{j}\right\}$. The likelihood for $a$ is

$$
L\left(a \mid \Omega_{j}\right)=\prod_{l=1}^{j} \psi^{y_{l}}\left(x_{l}, a\right)\left\{1-\psi\left(x_{l}, a\right)\right\}^{\left(1-y_{l}\right)} .
$$


The Bayesian framework of CRM assigns a prior distribution $f(a)$ for parameter $a$. We can generate the posterior distribution for $a$ by

$$
f\left(a \mid \Omega_{j}\right)=\frac{L\left(a \mid \Omega_{j}\right) f(a)}{\int_{a \in \mathcal{A}} L\left(a \mid \Omega_{j}\right) f(a) d a} .
$$

From the posterior distribution, we can generate an estimate $\hat{a}=\int_{a \in \mathcal{A}} a f\left(a \mid \Omega_{j}\right) d a$ by use of the posterior mean of $a$ and further obtain the estimates of the probability of toxicity at each dose level by $\hat{R}\left(d_{i}\right)=\psi\left(d_{i}, \hat{a}\right)$. Suppose $\theta$ is the target probability of DLT. The dose given to the $(j+1)$ th patient is the dose level $d_{i}$, which minimizes a loss function $\Delta\left(\hat{R}\left(d_{i}\right), \theta\right)$, The standard way is the absolute distance between $\hat{R}\left(d_{i}\right)$ and $\theta$ :

$$
\Delta\left(\hat{R}\left(d_{i}\right), \theta\right)=\left|\hat{R}\left(d_{i}\right)-\theta\right|
$$

which selects a dose whose estimated probability of toxicity is closest to the target toxicity $\theta$. Babb et al. (1998) proposed an asymmetric loss function that penalized probability of toxicity above the target probability more than that below the target probability. Specifically, a loss function is given by:

$$
\Delta\left(\hat{R}\left(d_{i}\right), \theta\right)= \begin{cases}\gamma\left(\theta-\hat{R}\left(d_{i}\right)\right) & \text { if } \theta>\hat{R}\left(d_{i}\right) \\ (1-\gamma)\left(\hat{R}\left(d_{i}\right)-\theta\right) & \text { if } \theta \leq \hat{R}\left(d_{i}\right)\end{cases}
$$

with $\gamma=0.5$ corresponding to a symmetric loss. The loss functions (1.4) and (1.5) illustrate the goal of the study. The overall goal of the study is also the goal for each patient enrolled in the study. The procedure continues until we have observed a predetermined sample size of patients or a stopping rule takes effect. The dose assigned to the hypothetical $(n+1) t h$ patient is the current best estimate for the MTD. The impact of the prior information tends to disappear in sequential 
estimation. Yet, because the sample size is usually very small in Phase I trials, reliance on prior information could be criticized. Therefore, O'Quigley and Shen (1996) further proposed a likelihood approach of CRM, which is a two-stage design similar to the original CRM.

The likelihood approach of CRM employs the same likelihood (1.2) as that for the original CRM. Using the expression of the likelihood, we may obtain an estimate for $a$ based on the maximum likelihood estimation. Once the estimate for $a$ has been calculated, the procedure continues in the same way as the original CRM. A requirement to achieve a maximum likelihood estimate in the interior of the parameter space is that we have observed some heterogeneity in the response, i.e. a toxicity is observed after a series of non-toxicities, or a non-toxicity is observed after a series of toxicities (Shen and O'Quigley, 1996). Otherwise, the maximum likelihood estimate is infinity, leading to a trivial estimate $\hat{R}\left(d_{i}\right)$, either zero, one or undefined, depending on the working model. O'Quigley and Shen (1996) proposed the use of a two-stage design with the first stage starting from the lowest dose and using an Up-and-Down escalation scheme, such as the traditional " $3+3$ " method, and with the second stage of maximum likelihood estimation triggered once heterogeneity in the response is achieved. The first stage varies with the choice of Up-and-Down escalation scheme. The choice of the scheme makes little difference because on average it is only used for a small portion in the experiments. If the scheme is reasonable, the operating characteristics of the two-stage design remain somewhat unaffected. In addition, O'Quigley and Shen (1996) compared the performance of the original CRM and the likelihood approach of CRM under a variety of scenarios. Generally, the two approaches yield quite similar operating characteristics in MTD estimation. 


\subsubsection{Partial Order CRM}

The CRM introduced by O'Quigley, Pepe and Fisher (1990) and O'Quigley and Shen (1996) was developed for a single-agent trial in which a higher dose corresponds to a larger probability of toxicity. In the language of Robertson, Wright and Dykstra (1998), the probability of toxicity follows a "simple order": the ordering of the probability of toxicity between any two doses is fully known and the higher dose corresponds to a larger probability of toxicity. For multiple-agent problems, it is reasonable to assume monotonicity for each agent separately, but, when combined, the ordering may not be fully known. For example, in Table 1.2, the ordering between treatment 3 and 4 is unknown. Some of the orderings between doses are known while others are not, which is a "partial order".

For multiple-agent problem, Robertson et al. (1988) described various possible partial orders scenarios. Hwang and Peddada (1994) proposed a method for estimating parameters subject partial orders that differentiated between "nodal" and restriction. Conaway, Dunbar, and Peddada (2004) proposed a two-stage Phase I design for partial orders. The first stage is designed to quickly escalate through dose combinations which are non-toxic. The second stage implements Hwang and Peddada's (1994) estimates. The true probability of toxicity for each treatment $i$ is modeled by $R\left(d_{i}\right), i=1, \ldots, k$. If prior information is available to investigators, it is described through a prior distribution of the form $R\left(d_{i}\right) \sim \operatorname{Beta}\left(\tau_{i}, \beta_{i}\right)$. The investigators specify the expected value of $R\left(d_{i}\right)$ and an upper limit $u_{i}$ such that it is $95 \%$ certain that the probability of toxicity will not exceed $u_{i}$.

$$
\mathrm{E}\left[R\left(d_{i}\right)\right]=\frac{\tau_{i}}{\tau_{i}+\beta_{i}} \quad \text { and } \quad \operatorname{Pr}\left[R\left(d_{i}\right) \leq u_{i}\right]=0.95
$$

The equations (1.6) are solved to obtain the parameters $\tau_{i}$ and $\beta_{i}$ in the prior. In the 
second stage, update the posterior means for the probability of toxicity subject to the partial order and compute a suggested treatment which minimizes the loss function associated with posterior means and target probability of DLT. In reality, Conaway, Dunbar, and Peddada's (2004) approach is complex and difficult to implement, especially for dose-finding studies that have a large number of possible simple orders.

Wages, Conaway and O'Quigley (2011) proposed a dose-finding design for multipleagent trials by laying out all possible simple orders that are consistent with the known orderings among dose combinations. When the ordering is fully known, it simplifies to the standard CRM introduced by O'Quigley, Pepe and Fisher (1990). The design is a Bayesian method. It begins by assigning a prior probability $p(m)$ for each possible ordering, where $m=1,2, \ldots, M, M$ is the number of possible orderings and $\sum_{m=1}^{M} p(m)=1$. To get the trial underway, the design selects an ordering according to the prior probability of each ordering and reduces the problem to a standard CRM to estimate the dose to treat the next patient. After having $j$ patients enrolled, the likelihood under $m$ is given by

$$
L_{m}\left(a_{m} \mid \Omega_{j}\right)=\prod_{l=1}^{j} \psi_{m}^{y_{l}}\left(x_{l}, a_{m}\right)\left\{1-\psi_{m}\left(x_{l}, a_{m}\right)\right\}^{\left(1-y_{l}\right)}
$$

which can be used to generate an estimate $\hat{a}_{m}$. The posterior probabilities of each ordering is updated by

$$
\pi\left(m \mid \Omega_{j}\right)=\frac{p(m) \int L_{m}\left(a \mid \Omega_{j}\right) f(a) d a}{\sum_{m=1}^{M} p(m) \int L_{m}\left(a \mid \Omega_{j}\right) f(a) d a}
$$

where $f(a)$ is the prior of $a$, and we generate an estimate $\hat{a}_{m}$ under each ordering $m$.

Later, Wages, Conaway and O'Quigley (2011) proposed a likelihood approach 
dose-finding design for multiple-agent trials. Similar to the Bayesian approach in 2010, the design is also based on each possible simple ordering. After having $j$ patients enrolled, the log-likelihood under the ordering $m$ is given by

$$
l_{m}\left(a_{m} \mid \Omega_{j}\right)=\sum_{l=1}^{j} y_{l} \log \psi_{m}\left(x_{l}, a_{m}\right)+\sum_{l=1}^{j}\left(1-y_{l}\right) \log \left\{1-\psi_{m}\left(x_{l}, a_{m}\right)\right\} .
$$

A plausible choice of weight for each ordering is

$$
\pi\left(m \mid \Omega_{j}\right)=\frac{\exp \left\{l_{m}\left(\hat{a}_{m} \mid \Omega_{j}\right)\right\}}{\sum_{m=1}^{M} \exp \left\{l_{m}\left(\hat{a}_{m} \mid \Omega_{j}\right)\right\}}
$$

where $\pi(m)$ is considered as the weight of evidence in favor of model $m$. Prior weights can be specified to down the weights of the orderings which are less plausible. With inclusion of prior weights $p(m)$ for each ordering, the generalized weights are

$$
\pi\left(m \mid \Omega_{j}\right)=\frac{\exp \left\{l_{m}\left(\hat{a}_{m} \mid \Omega_{j}\right)\right\} p(m)}{\sum_{m=1}^{M} \exp \left\{l_{m}\left(\hat{a}_{m} \mid \Omega_{j}\right)\right\} p(m)}
$$

The method sequentially updates the generalized weights for each possible ordering and generates an estimate $\hat{a}_{m}$ under each ordering in a likelihood approach. When a new patient is enrolled, the design selects an ordering according to the generalized weights and the problem reduces to a standard CRM to estimate the dose to treat the patient.

\subsubsection{Two Sample CRM}

There are relatively few statistical methods for designing dose-finding trials that consider heterogeneous toxicity response (e.g., Yuan, Chapell, 2004; Ivanova, Wang, 
2006; Thall, Nguyen, Estey, 2008 and Morita 2011). O'Quigley, Shen and Gamst (1999) discussed an extension of CRM which enabled the method to be applied to two groups of patients to determine the MTD for each group. The method takes the specification of a simple relationship between the dose-toxicity curves for each of the two groups and runs the two sample continual reassessment method using maximum likelihood.

O'Quigley and Paoletti (2003) investigated the two group continual reassessment method in which we anticipated some ordering between the two groups. In this situation, we may have quite strong knowledge concerning which of the two groups will have the higher level of MTD, if indeed they do not have the same MTD. However, the published methods are restricted to studies with at most two groups of patients.

\subsection{Outline of Dissertation}

This dissertation will address two practical considerations in dose-finding studies: heterogeneous toxicity response and a partial ordering for the probability of toxicity for available treatments. First, the majority of methods for the Phase I trials are designed for a homogeneous toxicity response, resulting in a unique MTD for the broad patient population. However, patients may naturally differ in the way they react to a treatment. In this dissertation, we consider heterogeneous toxicity response with a biomarker that is informative for the toxicity response. We propose a new design which chooses a distinct MTD for individual patient by use of toxicity biomarker information, thus contributing to a proper and better treatment for individual patient. Second, for multiple-agent trials we may be able to identify the order of the probability of toxicity for only a subset of the available treatments, which is a "par- 
tial order". We propose a biomarker-based design for which the ordering is not fully known. The standard CRM reviewed in Section 1.4.3 and the partial order CRM reviewed in Section 1.4.4 are in fact non-biomarker based desgins. We compare our biomarker-based designs with the traditional non-biomarker based designs.

Chapter 2 proposes a biomarker-based design for single-agent trial. The biomarker design for single-agent trial is in fact a biomarker design for simple order. It presents the toxicity probability model and the inference. It provides the simulation results comparing the new biomarker-based design with the standard CRM which is non-biomarker based. Furthermore, it proposes a BIC model selection method to choose a model between the biomarker model and the non-biomarker model.

Chapter 3 provides the theoretical properties of the biomarker design for simple order, including the convergence and asymptotic normality. The theoretical properties provide us confidence in using the proposed biomarker-based method.

Chapter 4 presents a biomarker-based design for multiple-agent Phase I trials, which is in fact a biomarker design for partial order. It shows the simulation results comparing the biomarker design for partial order with the biomarker design for fully known ordering and with partial order CRM. Furthermore, it discusses BIC model selection between the biomarker model and the non-biomarker model.

Chapter 5 finally concludes the dissertation with a discussion of the implications of the new biomarker-based designs together with ideas and areas for further research. 


\section{Chapter 2}

\section{Biomarker-based Design for}

\section{Single-agent Trial}

\subsection{Statistical Method}

\subsubsection{Toxicity Probability Model}

For single-agent trial, assume the probability of toxicity monotonically increases with the dose level, resulting in a "simple order". In general, we assume that there are $k$ pre-specified dose levels $d_{1}, d_{2}, \ldots, d_{k}$. Denote the dose for the $j$ th entered patient as $X_{j}, j=1,2, \ldots, n$, which takes value $x_{j} \in\left\{d_{1}, \ldots, d_{k}\right\}$. Suppose that a continuous biomarker $Z$ that is informative for toxicity outcome exists. Denote the biomarker of the $j$ th entered patient as $Z_{j} . Z_{j}$ is a random variable, but its value $z_{j}$ is known when the patient enters the trial. Denote $Y_{j}$ be an indicator for whether or not the $j t h$ entered patient experiences a DLT, which is a binary random variable, where 1 denotes the observation of a DLT and 0 denotes the observation of no DLT. We model the probability of toxicity $R\left(x_{j}, z_{j}\right)$ for the $j t h$ patient with 
two parameters $a$ and $b$ via

$$
R\left(x_{j}, z_{j}\right)=\operatorname{Pr}\left(Y_{j}=1 \mid X_{j}=x_{j}, Z_{j}=z_{j}\right)=E\left(Y_{j} \mid x_{j}, z_{j}\right)=\psi\left(x_{j}, z_{j}, a, b\right)
$$

for some working dose-toxicity model $\psi\left(x_{j}, z_{j}, a, b\right)$ and $a \in \mathcal{A}, b \in \mathcal{B}$.

For the assumption of homogeneous toxicity response, the standard CRM models the probability of toxicity $R\left(x_{j}\right)$ via $R\left(x_{j}\right)=\operatorname{Pr}\left(Y_{j}=1 \mid X_{j}=x\right)=E\left(Y_{j} \mid x_{j}\right)=$ $\psi\left(x_{j}, a\right)$ for some functional dose-toxicity curve $\psi\left(x_{j}, a\right)$ with one parameter $a$.

The power model $\psi\left(d_{i}, a\right)=\alpha_{i}^{a}$ or the empiric model $\psi\left(d_{i}, a\right)=\alpha_{i}^{\exp (a)}$, where $\alpha_{i}$ is a pre-specified skeleton (value) for each dose level $d_{i}$, is most commonly used working model (O'Quigley and Shen, 1996). $\psi\left(d_{i}, a\right)=\alpha_{i}^{\exp (a)}$ is a reparameterization form of the original power model $\psi\left(d_{i}, a\right)=\alpha_{i}^{a}$. In practice, the empiric model is preferred, because the domain of $a$ is all real numbers $\mathbf{R}$ in the reparameterization form while the domain of $a$ is positive real numbers $\mathbf{R}^{+}$in the power model.

For the assumption of heterogeneous toxicity response, we introduce the parameter $b$ to incorporate the biomarker to model the probability of toxicity. Based on the empiric model, we assign a linear relationship for the biomarker and explicit working dose-toxicity model:

$$
\psi\left(d_{i}, z, a, b\right)=\alpha_{i}^{\exp (a+b z)}
$$

where $\alpha_{i}$ are the pre-specified skeleton for each dose level $d_{i}$ and satisfy $0<\alpha_{1}<$ $\alpha_{2}<\cdots<\alpha_{k}<1$. This model is similar to the one used by O'Quigley, Shen and Gamst (1999) for the two-group CRM.

After inclusion of the first $j$ patients into the trial, we have data in the form of $\Omega_{j}=\left\{x_{1}, y_{1}, z_{1}, \ldots, x_{j}, y_{j}, z_{j}\right\}$. We generate estimates $\hat{a}$ and $\hat{b}$ for the parameters $a$ 
and $b$. Given the estimates $\hat{a}$ and $\hat{b}$, we can generate estimates of the probability of toxicity by $\hat{R}\left(d_{i}, z\right)=\psi\left(d_{i}, z, \hat{a}, \hat{b}\right)=\alpha_{i}^{\exp (\hat{a}+\hat{b} z)},(i=1, \ldots, k$ and $\forall z \in \mathcal{Z})$ for each of the $k$ treatments and any value of biomarker $z$.

Denote the pre-specified target toxicity probability as $\theta$. Suppose the biomarker of the $(j+1)$ th entered patient takes value $z_{j+1}$, the target dose to treat the patient has a corresponding probability of toxicity as close as possible to the target toxicity rate $\theta$. The treatment $x_{j+1}$ assigned to the $(j+1)$ th entered patient minimizes the loss function:

$$
\Delta\left(\hat{R}\left(d_{i}, z_{j+1}\right), \theta\right)=\left|\hat{R}\left(d_{i}, z_{j+1}\right)-\theta\right|, \quad i=1, \ldots, k
$$

which is the absolute distance between $\hat{R}\left(d_{i}, z_{j+1}\right)$ and $\theta$.

The design is a sequential updating design. After inclusion of a predetermined sample size of $n$ patients, the treatment assigned to the hypothetical $(n+1) t h$ patient with biomarker $z_{n+1}$ is the current best estimate for the MTD.

\subsubsection{Inference}

Similar to the Bayesian framework of O'Quigley, Pepe and Fisher (1990), we assign prior probability distributions $f_{0}(a)$ and $f_{1}(b)$ for the parameters $a$ and $b$.

In order to establish estimates of the probability of toxicity for each treatment, we need an expression of the joint likelihood for the parameters $a$ and $b$. After inclusion of the first $j$ patients into the study, the joint likelihood for the parameters $a$ and $b$ is given by:

$$
L\left(a, b \mid \Omega_{j}\right)=\prod_{l=1}^{j} \psi^{y_{l}}\left(x_{l}, z_{l}, a, b\right)\left\{1-\psi\left(x_{l}, z_{l}, a, b\right)\right\}^{\left(1-y_{l}\right)}
$$


which can be used to generate estimates $\hat{a}$ and $\hat{b}$ for $a$ and $b$.

Given the data set $\Omega_{j}$ and the joint likelihood, the posterior densities for $a$ and $b$ are given by:

$$
\begin{aligned}
f\left(a \mid \Omega_{j}\right) & =\frac{\int_{b \in \mathcal{B}} L\left(a, b \mid \Omega_{j}\right) f_{1}(b) d b}{\iint_{a \in \mathcal{A}, b \in \mathcal{B}} L\left(a, b \mid \Omega_{j}\right) f_{0}(a) f_{1}(b) d a d b} \\
f\left(b \mid \Omega_{j}\right) & =\frac{\int_{a \in \mathcal{A}} L\left(a, b \mid \Omega_{j}\right) f_{0}(a) d a}{\iint_{a \in \mathcal{A}, b \in \mathcal{B}} L\left(a, b \mid \Omega_{j}\right) f_{0}(a) f_{1}(b) d a d b} .
\end{aligned}
$$

The posterior mean can be used as the estimates for $a$ and $b$ :

$$
\begin{array}{r}
\hat{a}=\frac{\iint_{a \in \mathcal{A}, b \in \mathcal{B}} a L\left(a, b \mid \Omega_{j}\right) f_{0}(a) f_{1}(b) d a d b}{\iint_{a \in \mathcal{A}, b \in \mathcal{B}} L\left(a, b \mid \Omega_{j}\right) f_{0}(a) f_{1}(b) d a d b} \\
\hat{b}=\frac{\iint_{a \in \mathcal{A}, b \in \mathcal{B}} b L\left(a, b \mid \Omega_{j}\right) f_{0}(a) f_{1}(b) d a d b}{\iint_{a \in \mathcal{A}, b \in \mathcal{B}} L\left(a, b \mid \Omega_{j}\right) f_{0}(a) f_{1}(b) d a d b} .
\end{array}
$$

Given the working model $\psi\left(d_{i}, z, a, b\right)$ and estimates $\hat{a}$ and $\hat{b}$, we can generate estimates of probability of toxicity for each treatment and any value of biomarker. Beginning with prior probability distributions $f_{0}(a)$ and $f_{1}(b)$, after inclusion of the first $j$ patients into the trial, for the $(j+1)$ th enrolled patient with biomarker $z_{j+1}$ we can compute the posterior probability of DLT for each $d_{i}$ :

$$
\hat{R}\left(d_{i}, z_{j+1}\right)=\psi\left(d_{i}, z_{j+1}, \hat{a}, \hat{b}\right)
$$

The treatment $x_{j+1} \in\left\{d_{1}, \ldots, d_{k}\right\}$ assigned to the $(j+1)$ th enrolled patient is the treatment $d_{i}$, such that the loss function (2.3) is a minimum. For a patient with biomarker $z$, the MTD is the recommended treatment $d_{i}$ such that the loss function 
(2.3) is minimized after inclusion of the predetermined sample size of $n$ patients.

\subsection{Numerical Studies}

To illustrate the proposed method, consider an example involving $k=6$ treatments $d_{1}, \ldots, d_{6}$. We consider two examples of the true probability of toxicity.

In the first example, suppose the true probability of toxicity follows an empiric model:

$$
R\left(d_{i}, z\right)=\beta_{i}^{\exp (a+b z)}
$$

where $\beta_{1}=0.04, \beta_{2}=0.07, \beta_{3}=0.20, \beta_{4}=0.35, \beta_{5}=0.55$ and $\beta_{6}=0.70$. In this example, the form of the true probability of toxicity is similar to that of the working model.

In the second example, with the aim of further validating the robustness of the proposed design, we investigate the true probability of toxicity following a logit model:

$$
R\left(d_{i}, z\right)=\frac{\exp \left(a_{i}-b z\right)}{1+\exp \left(a_{i}-b z\right)}
$$

where $i=1, \ldots, 6$ and we study two scenarios of $a_{i}$, shown in Table 2.1.

Table 2.1: Scenarios of $a_{i}$

\begin{tabular}{ccccccc}
\hline & $a_{1}$ & $a_{2}$ & $a_{3}$ & $a_{4}$ & $a_{5}$ & $a_{6}$ \\
\hline Scenario 1 & -1.8 & -1.4 & -0.5 & 0.1 & 0.8 & 1.4 \\
Scenario 2 & -4.9 & -4 & -2.4 & -1.4 & -0.4 & 0.3 \\
\hline
\end{tabular}

The values of $a_{i}$ are taken such that for scenario $1, \beta_{i}^{\exp (-0.5)}=\frac{\exp \left(a_{i}\right)}{1+\exp \left(a_{i}\right)}, i=$ $1, \ldots, 6$ and for scenario $2, \beta_{i}^{\exp (0.42)}=\frac{\exp \left(a_{i}\right)}{1+\exp \left(a_{i}\right)}, i=1, \ldots, 6$, i.e. for biomarker with value 0 , the true probability of toxicity in the two examples are the same. In the second example, the form of the true probability of toxicity is different from that of 
the working model. The left plot in Figure 2.1 shows the true probability of toxicity $R\left(d_{i}, z\right)=\beta_{i}^{\exp (a+b z)}$ where $a=-0.5$ and $b=1$. The right plot in Figure 2.1 shows the true probability of toxicity $R\left(d_{i}, z\right)=\frac{\exp \left(a_{i}-b z\right)}{1+\exp \left(a_{i}-b z\right)}$ with $a_{i}$ in scenario 1 and $b=1$.
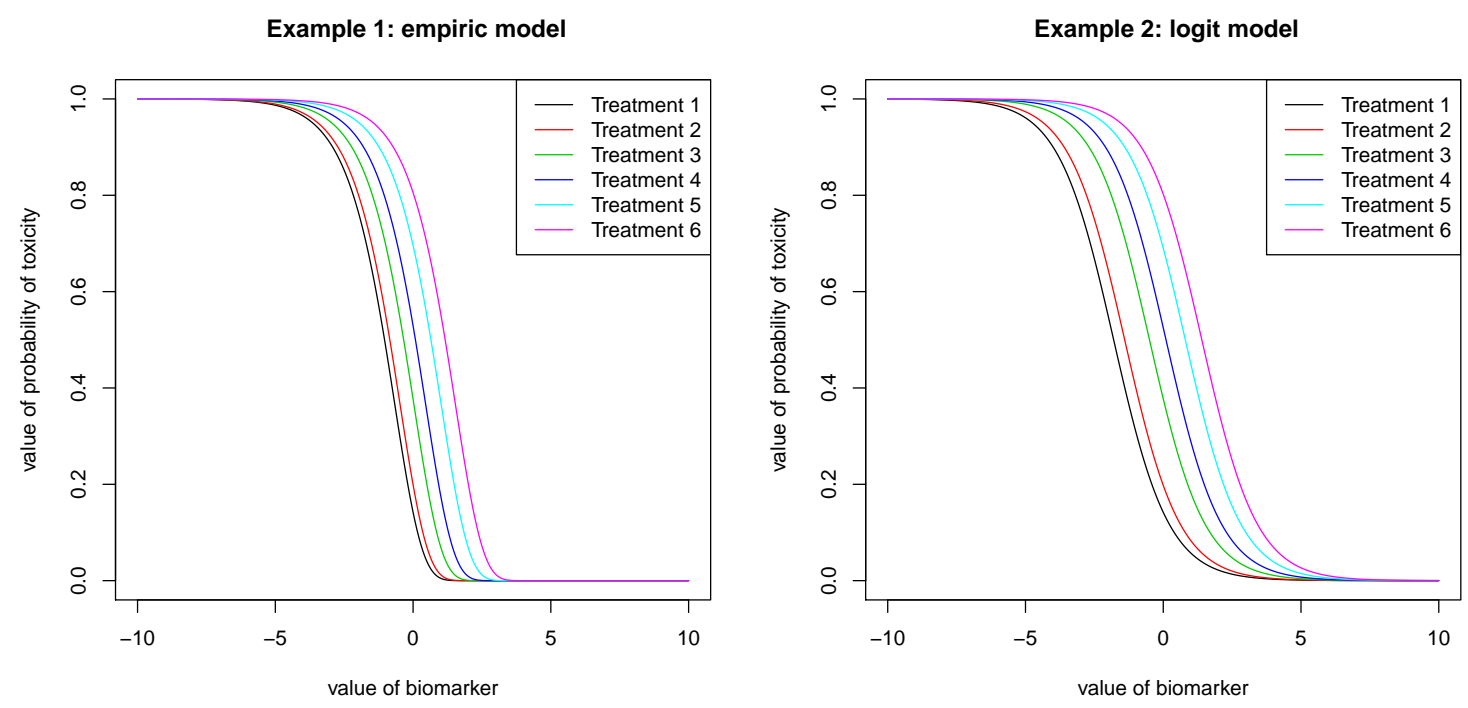

Figure 2.1: The true probability of toxicity with an empiric model and a logit model where $a=-0.5$ or $a_{i}$ in scenario 1 and $b=1$

Comparing the two plots, the empiric model is more steep than the logit model when the coefficients of the biomarker are the same in the two models. The empiric model is more sensitive to the change of the biomarker while the logit model is less sensitive to the change of the biomarker.

We investigate a variety of toxicity scenarios. For the true probability of toxicity with an empiric model, we study several different true values of $a$ and $b$, where $a=-0.5$ and 0.42 and $b=1.5,1,0.5,0.3,0.1,-0.1,-0.3,-0.5,-1$ and -1.5 . For the true probability of toxicity with a logit model, we study the two scenarios of $a_{i}$ in Table 2.1 and $b=1.5,1,0.5,0.3,0.1,-0.1,-0.3,-0.5,-1$ and -1.5 .

Suppose the target toxicity probability is $\theta=0.20$. 
There is a wide choice for the skeleton in the working dose-toxicity model $\psi\left(d_{i}, z, a, b\right)=\alpha_{i}^{\exp (a+b z)}$, where $0<\alpha_{1}<\alpha_{2}<\cdots<\alpha_{k}<1$. O'Quigley and Zohar (2010) indicated that "reasonable" skeletons should have adequate spacing between values at adjacent dose levels such that the operating characteristics of CRM will be adequate. Specifying skeletons with adequate spacing will be more challenging when the number of dose levels is large. Lee and Cheung (2009) established a systematic approach for specifying the skeleton. Their algorithm requires four parameters to generate the skeleton: the prior $\operatorname{MTD}(\gamma)$, the target toxicity probability $(\theta)$, the number of dose levels $(d)$ and the value of the indifference interval half-width $(\delta)$. It is necessary to ensure that there is enough spacing both below and above the prior MTD. Therefore, we recommend placing the prior MTD $(\gamma)$ at a dose level close to the middle dose levels. We can generate skeleton values using the getprior function in $\mathrm{R}$ package dfcrm (i.e., getprior $(\delta, \theta, \gamma, d)$ ). Using a half-width value of $\delta=0.07$, a prior MTD of $\gamma=3$ and a target toxicity probability $\theta=0.20$, implementing the algorithm yielded the skeleton $\{0.020,0.081,0.200,0.356,0.515$, $0.654\}$. We use this skeleton for the working model.

We assign a standard normal prior $N(0,1)$ for $a$ similar to O'Quigley and Shen (1996). Generally, before we begin the trial, we may have quite strong knowledge concerning whether an increased biomarker value indicates a higher probability of toxicity or a lower probability of toxicity. We assign a truncated normal prior for $b$. When we believe the probability of toxicity is decreasing with an increased biomarker value, corresponding to a true positive value of $b$ in the simulation, we assign a truncated normal prior for $b$ with the domain on $\mathbf{R}^{+}$:

$$
f_{1}(b)=\left\{\begin{array}{cc}
\frac{2}{\sqrt{2 \pi}} \exp \left(-\frac{b^{2}}{2}\right) & b \geq 0 \\
0 & b<0
\end{array}\right.
$$


When we believe the probability of toxicity is increasing with an increased biomarker value, corresponding to a true negative value of $b$ in the simulation, we assign a truncated normal prior for $b$ with the domain on $\mathbf{R}^{-}$:

$$
f_{1}(b)=\left\{\begin{array}{cc}
0 & b>0 \\
\frac{2}{\sqrt{2 \pi}} \exp \left(-\frac{b^{2}}{2}\right) & b \leq 0
\end{array}\right.
$$

We use a predetermined sample size of patients $n=25$ in the simulation. We define a measurement to evaluate the performance of the proposed design. After inclusion of the predetermined sample size of $n$ patients, for the hypothetical $(n+1)$ th patient with biomarker $z_{n+1}$, correct recommendation refers to the MTD recommended by the proposed design being the same as the true MTD corresponding to the value of biomarker. We further define the overall probability of correct recommendation as:

$$
\begin{aligned}
& P(\text { correct MTD recommendation }) \\
= & \int P(\text { correct MTD recommendation } \mid Z=z) f_{Z}(z) d z,
\end{aligned}
$$

where $f_{Z}(z)$ is the probability density function of biomarker $Z$.

To evaluate the operating characteristics and performance of the proposed method, we investigate biomarkers with different distributions. We first investigate a biomarker with a standard normal distribution $Z \sim N(0,1)$. According to genetic study, a toxicity biomarker may have a mixture distribution of a point mass at 0 and a distribution defined on $\mathbf{R}^{+}$. We then investigate biomarkers with such distributions. For the point mass at 0, we investigate two weights 0.3 and 0.6. For distributions defined on $\mathbf{R}^{+}$, we choose truncated normal distribution and log-normal distribution 
with $\mu=0$ and $\sigma=1$. The four specified mixture distributions are as follows:

$$
\begin{aligned}
& \mathrm{w}=0.3 \text { truncated normal } \\
& \operatorname{Pr}(Z=0)=0.3 \\
& f_{Z}(z)=0.7 \times \frac{2}{\sqrt{2 \pi}} \exp \left(-\frac{1}{2} z^{2}\right) \text { for } z>0 \\
& \mathrm{w}=0.6 \text { truncated normal } \\
& \operatorname{Pr}(Z=0)=0.6 \\
& f_{Z}(z)=0.4 \times \frac{2}{\sqrt{2 \pi}} \exp \left(-\frac{1}{2} z^{2}\right) \text { for } z>0 \\
& \operatorname{Pr}(Z=0)=0.3 \\
& \mathrm{w}=0.3 \log \text {-normal } \\
& f_{Z}(z)=0.7 \times \frac{1}{z \sqrt{2 \pi}} \mathrm{e}^{-\frac{(\ln z)^{2}}{2}} \text { for } z>0 \\
& \begin{array}{c}
\operatorname{Pr}(Z=0)=0.6 \\
f_{Z}(z)=0.4 \times \frac{1}{z \sqrt{2 \pi}} \mathrm{e}^{-\frac{(\ln z)^{2}}{2}} \text { for } z>0
\end{array} \\
& \mathrm{w}=0.6 \text { log-normal }
\end{aligned}
$$

When $b$ is positive, the probability of toxicity is decreasing with an increased biomarker value. When $b$ is negative, the probability of toxicity is increasing with an increased biomarker value. Figure 2.2 shows the probability of toxicity for a distribution on the whole real axis and the probability of toxicity for a truncated distribution on the positive semi axis.

Under all scenarios, 1000 trials were simulated. We compare our proposed biomarker design with the standard CRM whose working model does not contain biomarker although the true toxicity response is assumed to be heterogeneous in the simulation. In this dissertation, the "biomarker design for single-agent trial" proposed in Section 2.1 is synonymous with "biomarker design for simple order". The "standard CRM for single-agent trial" is synonymous with "non-biomarker design for simple order". 

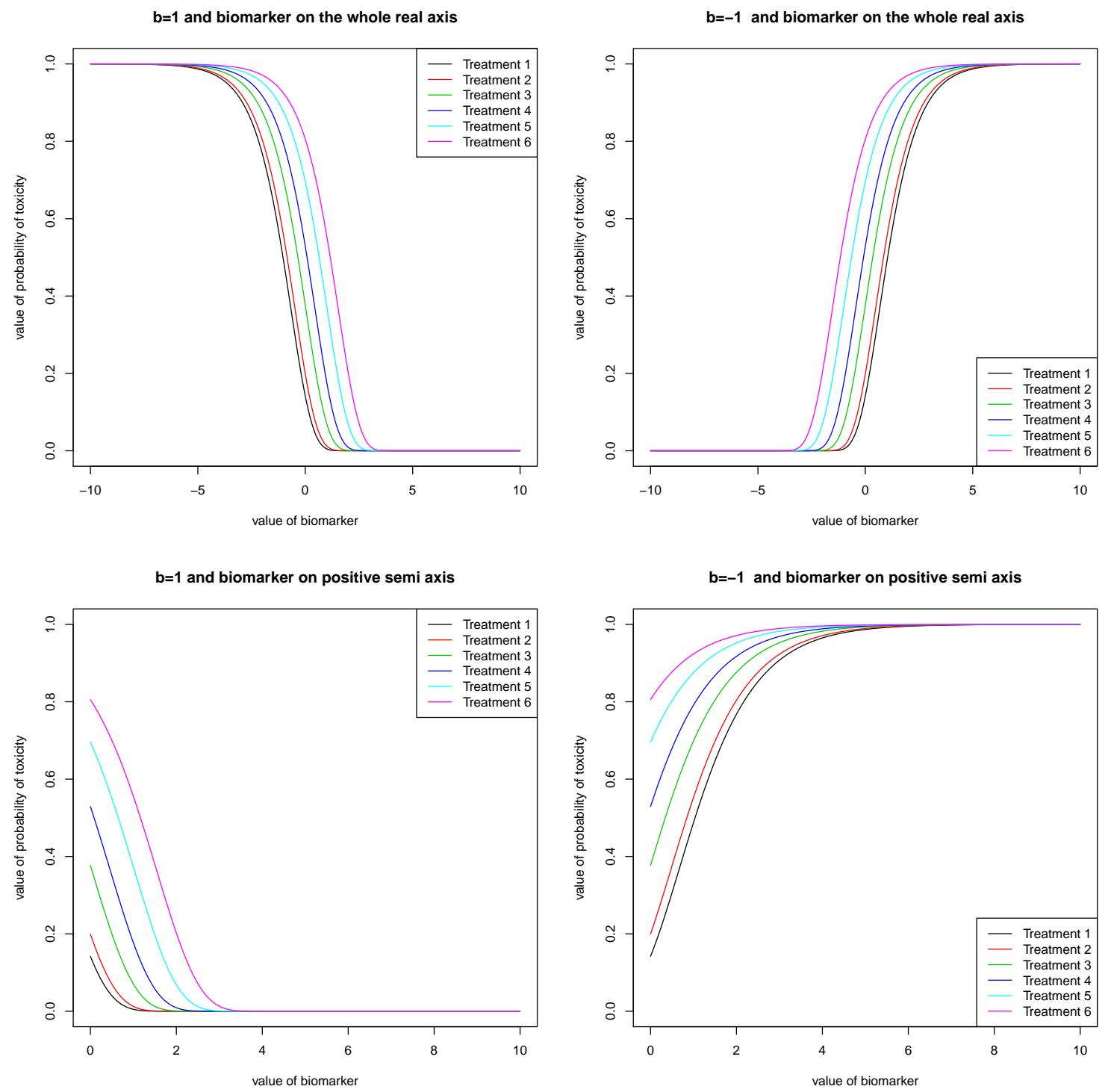

Figure 2.2: The true probability of toxicity on the whole real axis or on the positive semi axis where $a=-0.5$ and $b=1$ or $b=-1$

The standard CRM provides a benchmark of how necessary the biomarker information should be included and how well the proposed biomarker design performs. In the example of the true probability of toxicity with the empiric model (2.8), Figure A.1 to A.4 provide the probability of correct MTD recommendation. Figure A.5 to A.8 provide the corresponding percentage of patients treated at MTDs. 
In terms of the probability of correct MTD recommendation, when the biomarker is strong, corresponding to a large true absolute value of $b$ in the simulations, the proposed biomarker design yields a higher probability than the standard CRM. When the biomarker is weak, for example the scenarios with

(1) $a=-0.5, b=0.1$ and biomarker with truncated normal $w=0.3$

(2) $a=-0.5, b=0.1$ and biomarker with truncated normal $w=0.6$

(3) $a=-0.5, b=0.1$ and biomarker with log-normal $w=0.3$

(4) $a=-0.5, b=0.1$ and biomarker with log-normal $w=0.6$

(5) $a=0.42, b=0.5$ and biomarker with truncated normal $w=0.6$

(6) $a=0.42, b=0.3$ and biomarker with truncated normal $w=0.3$

(7) $a=0.42, b=0.3$ and biomarker with truncated normal $w=0.6$

(8) $a=0.42, b=0.3$ and biomarker with log-normal $w=0.6$

(9) $a=0.42, b=0.1$ and biomarker with standard normal distribution

(10) $a=0.42, b=0.1$ and biomarker with truncated normal $w=0.3$

(11) $a=0.42, b=0.1$ and biomarker with truncated normal $w=0.6$

(12) $a=0.42, b=0.1$ and biomarker with log-normal $w=0.3$

(13) $a=0.42, b=0.1$ and biomarker with log-normal $w=0.6$

(14) $a=0.42, b=-0.1$ and biomarker with standard normal distribution

(15) $a=0.42, b=-0.1$ and biomarker with truncated normal $w=0.3$ 
(16) $a=0.42, b=-0.1$ and biomarker with truncated normal $w=0.6$

(17) $a=0.42, b=-0.1$ and biomarker with log-normal $w=0.3$

(18) $a=0.42, b=-0.1$ and biomarker with log-normal $w=0.6$

standard CRM yields slightly higher probability than the proposed biomarker design. Yet, the probability of correct MTD recommendation yielded by the proposed biomarker design is also high enough.

In terms of the percentage of patients treated at the MTDs, when the biomarker is strong, corresponding to a large true absolute value of $b$ in the simulations, the proposed biomarker design treats more patients at the MTDs than the standard CRM. When the biomarker is weak, for example the scenarios with

(1) $a=-0.5, b=0.3$ and biomarker with truncated normal $w=0.3$

(2) $a=-0.5, b=0.3$ and biomarker with truncated normal $w=0.6$

(3) $a=-0.5, b=0.1$ and biomarker with truncated normal $w=0.3$

(4) $a=-0.5, b=0.1$ and biomarker with truncated normal $w=0.6$

(5) $a=-0.5, b=0.1$ and biomarker with log-normal $w=0.3$

(6) $a=-0.5, b=0.1$ and biomarker with log-normal $w=0.6$

(7) $a=0.42, b=0.3$ and biomarker with truncated normal $w=0.3$

(8) $a=0.42, b=0.3$ and biomarker with truncated normal $w=0.6$

(9) $a=0.42, b=0.3$ and biomarker with log-normal $w=0.6$

(10) $a=0.42, b=0.1$ and biomarker with standard normal distribution 
(11) $a=0.42, b=0.1$ and biomarker with truncated normal $w=0.3$

(12) $a=0.42, b=0.1$ and biomarker with truncated normal $w=0.6$

(13) $a=0.42, b=0.1$ and biomarker with log-normal $w=0.3$

(14) $a=0.42, b=0.1$ and biomarker with log-normal $w=0.6$

(15) $a=0.42, b=-0.1$ and biomarker with standard normal distribution

(16) $a=0.42, b=-0.1$ and biomarker with truncated normal $w=0.3$

(17) $a=0.42, b=-0.1$ and biomarker with truncated normal $w=0.6$

(18) $a=0.42, b=-0.1$ and biomarker with log-normal $w=0.3$

(19) $a=0.42, b=-0.1$ and biomarker with log-normal $w=0.6$

standard CRM treats slightly more patients at the MTDs than the proposed biomarker design.

The simulation results indicate that the proposed biomarker design is not quite sensitive to the distribution of biomarker. In the example of the true probability of toxicity with the logit model (2.9), Figure A.5 to A.8 provide the probability of correct MTD recommendation. Figure A.13 to A.16 provide the corresponding percentage of patients treated at MTDs. Similarly, when the biomarker is strong, the proposed biomarker design performs better than the standard CRM. When the biomarker is weak, the standard CRM may perform slightly better than the proposed biomarker design. It shows the proposed biomarker design is not quite sensitive to the underlying true probability of toxicity and demonstrates the robustness of the method. 
Overall, the simulation results indicate that, in terms of identifying MTDs, the proposed biomarker design demonstrates superior performance when the biomarker is strong and the performance of the proposed biomarker design is comparable to that of standard CRM when the biomarker is weak. The simulation results support the feasibility and necessity of choosing a distinct MTD for each individual patient by use of toxicity biomarker.

\subsection{Model Selection between Biomarker Model and Non-biomarker Model}

\subsubsection{Statistical Method}

The simulation result in Section (2.2) indicates that when the biomarker is strong the biomarker design proposed in section (2.1) performs better, when the biomarker is weak the standard CRM, i.e. the non-biomarker model, performs better.

When the toxicity response is homogeneous, O'Quigley, Pepe and Fisher (1990) pointed out a one-parameter working model such as $\psi\left(d_{i}, a\right)=\alpha_{i}^{\exp (a)}$ is a underparameterized model. They compared the performance of a one-parameter model versus with a two-parameter model. Generally, the one-parameter model performs better than a two-parameter model.

When the toxicity response is heterogenous, the biomarker model is more flexibility than the non-biomarker model in identifying the MTDs. The non-biomarker model is relatively under-parameterized. It chooses a unique MTD for all values of biomarker. The non-biomarker model leads to more handicap in trying to find a good fit and a correct MTD for some values of biomarker. However, when the biomarker is weak, most values of biomarker may have the same MTD. The lack of 
flexibility of the non-biomarker model may turn into an advantage in identifying a correct MTD for most values of biomarker, especially considering the small sample size of patients and vague prior of the parameters. The non-biomarker model may be considered as the better model than the biomarker model when the biomarker is weak. When the biomarker is strong, the correct MTDs spread out for different values of biomarker, so the biomarker model is considered better while the nonbiomarker model is lack of flexibility.

This section has the appeal of implementing model selection techniques. If the model selection technique can effectively choose the better model between the biomarker model and the non-biomarker model, our approach will identify MTDs more effectively. Schwarz (1978) derived the Bayesian information criterion (BIC) given by:

$$
B I C=-2 \log \left(L\left(\mathbf{a} \mid \Omega_{j}\right)\right)+K \log (n)
$$

where $\mathbf{a}$ is the estimable parameters and $K$ is the number of estimable parameters. In the proposed biomarker design, two parameters $a_{b i o}$ and $b_{b i o}$ are estimated, $K=2$ and the BIC is given by:

$$
B I C_{b i o}=-2 \log \left(L\left(\hat{a}_{b i o}, \hat{b}_{b i o} \mid \Omega_{j}\right)\right)+2 \log (n) .
$$

The expression $L\left(\hat{a}_{b i o}, \hat{b}_{b i o} \mid \Omega_{j}\right)$ is the value of the likelihood evaluated at the point of posterior mean $\hat{a}_{b i o}$ and $\hat{b}_{b i o}$. In the non-biomarker design, the working model is $\psi\left(d_{i}, a\right)=\alpha_{i}^{\exp (a)}$, one parameter $a_{\text {non }}$ is estimated, $K=1$ and the BIC is given by:

$$
B I C_{\text {non }}=-2 \log \left(L\left(\hat{a}_{\text {non }} \mid \Omega_{j}\right)\right)+\log (n)
$$

The expression $L\left(\hat{a}_{n o n} \mid \Omega_{j}\right)$ is the value of the likelihood evaluated at the posterior 
mean $\hat{a}_{\text {non }}$. The model with smaller BIC is considered better and will be selected.

Before the first patient is enrolled, we do not have the estimates $\hat{a}_{b i o}, \hat{b}_{b i o}$ and $\hat{a}_{\text {non }}$. We simply choose the non-biomarker model as the working model. When the estimates $\hat{a}_{b i o}, \hat{b}_{b i o}$ and $\hat{a}_{n o n}$ are all achieved, we are able to compute and compare the BIC for the biomarker model and the non-biomarker model. In the Bayesian framework, we obtain the posterior mean $\hat{a}_{b i o}, \hat{b}_{b i o}$ and $\hat{a}_{n o n}$ as soon as the response of whether or not the first patient experiences a DLT is observed. After that, the BIC is computed for the biomarker model and the non-biomarker model and the model with the smaller value of BIC is selected.

After inclusion of the first $j$ patients into the trial, we estimate both the biomarker model and the non-biomarker model and generate estimates $\hat{a}_{b i o}, \hat{b}_{b i o}$ and $\hat{a}_{n o n}$. When the $(j+1)$ th patient is enrolled, select the model with smaller BIC between the biomarker model $\psi\left(d_{i}, z, a, b\right)=\alpha_{i}^{\exp (a+b z)}$ and the non-biomarker model $\psi\left(d_{i}, a\right)=\alpha_{i}^{\exp (a)}$, and then select the treatment $x_{j+1}$ assigned to the $(j+1)$ th entered patient which minimizes the loss function (2.3). After inclusion of a predetermined sample size of $n$ patients, we choose the model with smaller BIC as the working model and choose the MTDs for each individual patient under the working model.

\subsubsection{Numerical Studies}

In aim of improving the performance of biomarker model when the biomarker is weak, we propose the model selection method. In this section, we compare the performance of the BIC model selection method with the biomarker model and the non-biomarker model. In this dissertation, the "BIC model selection method" proposed in Section (2.3) is synonymous with "BIC method for simple order". We 
do the simulation studies under the same setting as Section (2.2).

In the example of the true probability of toxicity with the empiric model (2.8), Figure A.1 to A.4 provide the probability of correct MTD recommendation. Figure A.5 to A.8 provide the corresponding percentage of patients treated at MTDs. Table A.1 provides the proportion of selecting biomarker model as the final working model after inclusion of the predetermined sample size of patients. In the example of the true probability of toxicity with the logit model (2.9), Figure A.5 to A.8 provide the probability of correct MTD recommendation. Figure A.13 to A.16 provide the corresponding percentage of patients treated at MTDs. Table A.2 provides the proportion of selecting biomarker model as the final working model

In terms of the probability of correct MTD recommendation, the probability of the BIC method is usually between the probability of the biomarker model and the non-biomarker model. When the biomarker is strong, the probability of biomarker model is larger than the non-biomarker model, the probability of the BIC method is usually slightly smaller than but approaches to that of biomarker model. It is reasonable that when the probability of biomarker model is larger than that of the non-biomarker model, the probability of the BIC method is smaller than that of biomarker model, because with the model selection mechanism the BIC method sometimes chooses the non-biomarker model, further lowering the probability of correct MTD recommendation.

When the biomarker is weak, the probability of biomarker model is sometimes smaller than that of the non-biomarker model. Especially, for those scenarios whose probability of biomarker model is smaller than that of non-biomarker model, the probability of BIC method is obviously larger than that of biomarker model and approaches to the probability of non-biomarker model. We conclude that the BIC method generally improves the performance in identifying MTDs when the biomark- 
er is weak.

Similarly for the percentage of patients treated at the MTDs, when the biomarker is strong, the percentage of BIC method is usually slightly smaller than but approaches to that of biomarker model. When the biomarker is weak, the BIC method usually improves the percentage.

In terms of the proportion of selecting biomarker model as the final working model, when the biomarker is strong, the proportion is large, when the biomarker is weak, the proportion could be very small. In addition, the proportion has some certain pattern which is related to the distribution of the biomarker.

Overall, the BIC method is a trade off between biomarker model and nonbiomarker model. When the biomarker is strong, the BIC method may lower the performance a little bit. When the biomarker is weak, the BIC method improves the performance a lot. The BIC method generates a good result much more stably than the biomarker model and non-biomarker model whenever the biomarker is strong or weak. The simulation results indicate that it is worthwhile to replace the biomarker model with the BIC model selection method, especially when it is uncertain whether the biomarker is strong or not before the trial. Yet when it is quite certain that the biomarker is strong before the trial, the biomarker model is still recommended. 


\section{Chapter 3}

\section{Theoretical Properties}

For the homogeneous toxicity response, Shen and O'Quigley (1996) established the large sample properties including consistency and asymptotic normality under model misspecification. It is shown that, in general, the recommended dose level will converge to the target dose level. However under model misspecification there are situations in which the recommended dose may converge to a dose level close to the target dose level, but not necessarily the closest one. O'Quigley (2005) summarized the theoretical study of the continual reassessment method. Cheung and Chappell (2002) conjectured that CRM is consistent under a much weaker set of conditions than the conditions established by Shen and O'Quigley (1996). Azriel (2012) provided a formal proof for the conjecture of Cheung and Chappell (2002), thus giving a solid justification for the robustness of the CRM for misspecified model.

O'Quigley, Shen and Gamst (1999) proved the consistency and asymptotic normality for two sample CRM under fairly weak conditions and established the asymptotic efficiency between two sample CRM and two one-sample CRM separately. For partial order CRM, Wages (2010) developed the theoretical properties in his unpublished Ph.D. thesis, within the framework of stochastic approximation and proved 
consistent model selection among each possible simple order.

All the arguments are acquired from the idea of the likelihood and based on maximum likelihood estimation. As long as the probability of the prior distribution for the parameters to be estimated is not placed on a single point, the arguments hold for the Bayesian approach as well. Common arguments involving the likelihood for completely random dose allocation fail due to the determinant dose allocation. For the heterogeneous toxicity response, we also proceed from the likelihood and maximum likelihood estimation. Therefore, we define the following functions:

$$
\begin{aligned}
L_{n}(a, b)= & \prod_{j=1}^{n}\left\{\psi^{y_{l}}\left(x_{j}, z_{j}, a, b\right)\right\}^{y_{j}}\left\{1-\psi\left(x_{j}, z_{j}, a, b\right)\right\}^{\left(1-y_{j}\right)} \\
l_{n}(a, b)= & \sum_{j=1}^{n}\left[y_{j} \log \left\{\psi\left(x_{j}, z_{j}, a, b\right)\right\}+\left(1-y_{j}\right) \log \left\{1-\psi\left(x_{j}, z_{j}, a, b\right)\right\}\right] \\
I_{n}(a, b)= & \frac{1}{n} \sum_{j=1}^{n}\left[y_{j} \log \left\{\psi\left(x_{j}, z_{j}, a, b\right)\right\}+\left(1-y_{j}\right) \log \left\{1-\psi\left(x_{j}, z_{j}, a, b\right)\right\}\right. \\
\tilde{L}_{n}(a, b)= & \prod_{j=1}^{n}\left\{\psi\left(x_{j}, z_{j}, a, b\right)\right\}{ }^{R\left(x_{j}, z_{j}\right)}\left\{1-\psi\left(x_{j}, z_{j}, a, b\right)\right\}^{\left\{1-R\left(x_{j}, z_{j}\right)\right\}} \\
\tilde{l}_{n}(a, b)= & \sum_{j=1}^{n}\left[R\left(x_{j}, z_{j}\right) \log \left\{\psi\left(x_{j}, z_{j}, a, b\right)\right\}\right. \\
& \left.+\left\{1-R\left(x_{j}, z_{j}\right)\right\} \log \left\{1-\psi\left(x_{j}, z_{j}, a, b\right)\right\}\right] \\
& +\frac{1}{n} \sum_{j=1}^{n}\left[R\left(x_{j}, z_{j}\right) \log \left\{\psi\left(x_{j}, z_{j}, a, b\right)\right\}\right. \\
& \left.+\left\{1-R\left(x_{j}, z_{j}\right)\right\} \log \left\{1-\psi\left(x_{j}, z_{j}, a, b\right)\right\}\right]
\end{aligned}
$$

where $R\left(d_{i}, z\right)$ is the true probability of toxicity for dose level $d_{i}$ and biomarker $z, \psi\left(d_{i}, z, a, b\right)$ is the working model and $y_{j}$ is the toxicity response for the $j t h$ patient. $L_{n}(a, b)$ is the likelihood, $l_{n}(a, b)$ is the log-likelihood. Substituting the true probability of toxicity $R\left(x_{j}, z_{j}\right)$ for the toxicity response $y_{j}$, we get the likelihood 
$\tilde{L}_{n}(a, b)$ and the log-likelihood $\tilde{l}_{n}(a, b)$.

The maximum likelihood estimation $\hat{a}_{n}$ and $\hat{b}_{n}$ exist as soon as some kind heterogeneity in the toxicity response is achieved. We need to assume the working model $\psi\left(d_{i}, z, a, b\right)$ to satisfy the following conditions in order to establish the useful properties including the consistency and asymptotic normality.

Condition 1. The parameter a belongs to a finite interval $\left[m_{a}, M_{a}\right]$ and the parameter $b$ belongs to a finite interval $\left[m_{b}, M_{b}\right]$. The biomarker $z \in Z$ and the domain of $z$ is bounded in a finite interval $\left[m_{z}, M_{z}\right]$. The biomarker $z$ is not a point mass, i.e. for any $c, P(Z=c)<1$.

This restriction enables us to avoid dealing with degenerate cases (the probability of toxicity equaling to zero or one).

Condition 2. For fixed $z$, the probability of toxicity satisfy: $0<R\left(d_{1}, z\right)<\cdots<$ $R\left(d_{k}, z\right)<1$ and there exists $m_{R}$ and $M_{R}$ such that for all $z, 0<m_{R} \leq R\left(d_{1}, z\right)<$ $\cdots<R\left(d_{k}, z\right) \leq M_{R}<1$. For each $d_{i}$, the probability of toxicity $R\left(d_{i}, z\right)$ is monotone in $z$.

The restriction enables us to avoid dealing with degenerate cases (true probability of toxicity equaling to zero or one). The restriction accords with the assumption that the higher dose corresponds to a larger probability of toxicity. The restriction indicates that the biomarker is monotonic with the true probability of toxicity.

Condition 3. $\psi(x, z, a, b)$ can be written as a function of $x$ and $a+b z$, i.e. $\psi(x, z, a, b)$ $=\tilde{\psi}(x, a+b z)$. For each $d_{i}$, if $\psi\left(d_{i}, z, a_{1}, b_{1}\right)=\psi\left(d_{i}, z, a_{2}, b_{2}\right), a_{1}+b_{1} z=a_{2}+b_{2} z$.

The condition $\psi\left(d_{i}, z, a, b\right)$ can be written as a function of $a+b z$ comes from the motivation of the proposed working model. For the assumption of homogeneous 
toxicity response, we usually use a one-parameter working model. For the assumption of heterogeneous toxicity response, to incorporate the biomarker information, we assign a linear relationship for the biomarker. It is in the form of $a+b z$, instead of the single parameter $a$ for the homogeneous assumption.

The empiric model $\psi\left(d_{i}, z, a, b\right)=\alpha_{i}^{\exp (a+b z)}$ proposed in Section (2.1) satisfies this condition. It can be written as a function of $a+b z$. If $\alpha_{i}^{\exp \left(a_{1}+b_{1} z\right)}=\alpha_{i}^{\exp \left(a_{2}+b_{2} z\right)}$ , we will have $a_{1}+b_{1} z=a_{2}+b_{2} z$.

In addition to the empiric model, there exists other function satisfying the condition 3. For example, a logit model $\psi\left(d_{i}, z, a, b\right)=\frac{\exp (a+b z)}{1+\exp (a+b z)}$ satisfies the condition 3.

Condition 4. $\psi(x, z, a, b)$ is continuous.

Condition 5. For fixed $z, a$ and $b, \psi(x, z, a, b)$ is strictly increasing in $x$.

Condition 6. For fixed $x, a$ and $b, \psi(x, z, a, b)$ is strictly monotonic in $z$.

Condition 7. $\psi(x, z, a, b)$ is second order continuous derivable about a and $b$.

Proposition 1. (The uniform continuity of $\psi(\cdot)) \forall \varepsilon>0$, there exists $\delta_{a}>0$, $\delta_{b}>0$, for $\left|a_{1}-a_{2}\right|<\delta_{a},\left|b_{1}-b_{2}\right|<\delta_{b}, \forall z \in\left[m_{z}, M_{z}\right]$ and $\forall i=1, \ldots, k$,

$$
\left|\psi\left(d_{i}, z, a_{1}, b_{1}\right)-\psi\left(d_{i}, z, a_{2}, b_{2}\right)\right|<\varepsilon
$$

holds.

Proof. Since $a \in\left[m_{a}, M_{a}\right], b \in\left[m_{b}, M_{b}\right]$ and $z \in\left[m_{z}, M_{z}\right], \psi\left(d_{i}, z, a, b\right)$ is uniformly continuous, the proposition holds.

Proposition 2. (The continuity of log-likelihood) For each $\varepsilon>0$ and every $(a, b) \in\left[m_{a}, M_{a}\right] \times\left[m_{b}, M_{b}\right]$ and $z \in Z \subset\left[m_{z}, M_{z}\right]$, there exists $N_{\varepsilon}$ such that whenever 
$n>N_{\varepsilon}$, we have

$$
\left|I_{n}(a, b)-I_{n+1}(a, b)\right|<\varepsilon
$$

Proof. For some $M>0$ and for each $n>M$, we can write

$$
I_{n}(a, b)=\frac{M}{n} I_{M}(a, b)+\frac{1}{n} \sum_{j=1}^{n-M} \rho_{M+j}
$$

where $\rho_{j}=\log \left\{\psi\left(x_{j}, z_{j}, a, b\right)\right\}$ if the $j$ th patient with biomarker $z_{j}$ experienced a toxicity at dose $x_{j}$ and $\rho_{j}=\log \left\{1-\psi\left(x_{j}, z_{j}, a, b\right)\right\}$ otherwise. Now let $D_{1}$ and $D_{2}$ be bounds such that $\left|I_{M}(a, b)\right|<D_{1}$ and $\left|\rho_{j}\right|<D_{2}$. Next, if $N_{\varepsilon}=\max \left(\frac{2 D_{1}}{M \varepsilon}, \frac{2 D_{2}}{\varepsilon}\right)$ and $n>N_{\varepsilon}$, then we have

$$
\begin{aligned}
& \left|I_{n}(a, b)-I_{n+1}(a, b)\right| \\
= & \left|\frac{M}{n} I_{M}(a, b)+\frac{1}{n} \sum_{j=1}^{n-M} \rho_{M+j}-\frac{M}{n+1} I_{M}(a, b)-\frac{1}{n+1} \sum_{j=1}^{n-M+1} \rho_{M+j}\right| \\
= & \left|\frac{M}{n(n+1)} I_{M}(a, b)+\frac{1}{n(n+1)} \sum_{j=1}^{n-M} \rho_{M+j}-\frac{1}{n+1} \rho_{n+1}\right| \\
< & \frac{M}{n(n+1)} D_{1}+\frac{n-M+1}{n(n+1)} D_{2} \leq \frac{M}{n(n+1)} D_{1}+\frac{1}{n} D_{2}<\frac{\varepsilon}{2}+\frac{\varepsilon}{2}=\varepsilon .
\end{aligned}
$$

The last inequality follows from our choice of $N_{\varepsilon}$.

Corollary 1. Similar inequalities

$$
\begin{array}{r}
\left|\tilde{I}_{n}(a, b)-\tilde{I}_{n+1}(a, b)\right|<\varepsilon \\
\left|\frac{\partial I_{n}(a, b)}{\partial a}-\frac{\partial I_{n+1}(a, b)}{\partial a}\right|<\varepsilon \\
\left|\frac{\partial I_{n}(a, b)}{\partial b}-\frac{\partial I_{n+1}(a, b)}{\partial b}\right|<\varepsilon
\end{array}
$$

hold. 
Lemma 1. If $S_{n}=\sum_{i=1}^{n} X_{i}$ is a zero-mean martingale and $E X_{i}^{2}$ is uniformly bounded for all $i$, we have

$$
\frac{S_{n}}{n} \rightarrow 0 \text { almost surely. }
$$

Proof. It is obvious that

$$
\begin{gathered}
E X_{j}=E\left(E\left(X_{j} \mid F_{j-1}\right)\right)=E\left(E\left(\left(S_{j}-S_{j-1}\right) \mid F_{j-1}\right)\right)=E\left(E\left(S_{j} \mid F_{j-1}\right)-S_{j-1}\right)=0, \\
E S_{n}=0 .
\end{gathered}
$$

For $i>j$,

$E\left(X_{i} X_{j}\right)=E\left(E\left(X_{i} X_{j} \mid F_{i-1}\right)\right)=E\left(X_{j} E\left(X_{i} \mid F_{i-1}\right)\right)=E\left(X_{j}\left(E\left(S_{i} \mid F_{i-1}\right)-S_{i-1}\right)\right)=0$.

Thus, we have

$$
\operatorname{var} S_{n}=E S_{n}^{2}=\sum_{j=1}^{n} E X_{j}^{2}+2 \sum_{i>j} E\left(X_{i} X_{j}\right)=\sum_{j=1}^{n} E X_{j}^{2}
$$

Consider $Y_{j}=\frac{X_{j}}{j}$ and the series $\tilde{S}_{n}=\sum_{j=1}^{n} Y_{j}=\sum_{j=1}^{n} \frac{X_{j}}{j}$.

$\tilde{S}_{n}$ is also a martingale and $E Y_{j}=0, E \tilde{S}_{n}=0, E\left(Y_{i} Y_{j}\right)=0, \operatorname{var} \tilde{S}_{n}=\sum_{j=1}^{n} E Y_{j}^{2}$.

First, for $\forall \varepsilon>0$, we show $P\left(\max _{1 \leq k \leq n}\left|\tilde{S}_{k}\right| \geq \varepsilon\right) \leq \frac{\sum_{j=1}^{n} E Y_{j}^{2}}{\varepsilon^{2}}$.

Define

$$
\begin{aligned}
A & =\left\{\max _{1 \leq k \leq n}\left|\tilde{S}_{k}\right| \geq \varepsilon\right\}=\bigcup_{j=1}^{n}\left\{\left|\tilde{S}_{j}\right| \geq \varepsilon\right\} \\
& =\bigcup_{k=1}^{n}\left\{\left|\tilde{S}_{1}\right|<\varepsilon, \cdots,\left|\tilde{S}_{k-1}\right|<\varepsilon,\left|\tilde{S}_{k}\right| \geq \varepsilon\right\}=\bigcup_{k=1}^{n} A_{k}
\end{aligned}
$$


Define $A_{k}=\left\{\left|\tilde{S}_{1}\right|<\varepsilon, \cdots,\left|\tilde{S}_{k-1}\right|<\varepsilon,\left|\tilde{S}_{k}\right| \geq \varepsilon\right\}$, so $A_{k}$ are disjoint events.

We have

$$
P(A)=\sum_{k=1}^{n} P\left(A_{k}\right)
$$

and

$$
\begin{gathered}
\operatorname{var}\left(\tilde{S}_{n}\right)=E \tilde{S}_{n}^{2} \geq E\left(\tilde{S}_{n}^{2} I_{A}\right)=\sum_{k=1}^{n} E\left(\tilde{S}_{n}^{2} I_{A_{k}}\right)=\sum_{k=1}^{n} E\left[\left(\tilde{S}_{k}+\tilde{S}_{n}-\tilde{S}_{k}\right)^{2} I_{A_{k}}\right] \\
=\sum_{k=1}^{n} E\left(\tilde{S}_{k}^{2} I_{A_{k}}\right)+2 \sum_{k=1}^{n} E\left[\tilde{S}_{k}\left(\tilde{S}_{n}-\tilde{S}_{k}\right) I_{A_{k}}\right]+\sum_{k=1}^{n} E\left[\left(\tilde{S}_{n}-\tilde{S}_{k}\right)^{2} I_{A_{k}}\right] .
\end{gathered}
$$

In the cross term,

$$
\begin{aligned}
& E\left[\tilde{S}_{k}\left(\tilde{S}_{n}-\tilde{S}_{k}\right) I_{A_{k}}\right]=E\left[\tilde{S}_{k} I_{A_{k}} \sum_{j=k+1}^{n} Y_{j}\right]=\sum_{j=k+1}^{n} E\left(\tilde{S}_{k} I_{A_{k}} Y_{j}\right) \\
= & \sum_{j=k+1}^{n} E\left(E\left(\tilde{S}_{k} I_{A_{k}} Y_{j} \mid F_{j-1}\right)\right)=\sum_{j=k+1}^{n} E\left[\tilde{S}_{k} I_{A_{k}} E\left(\left(\tilde{S}_{j}-\tilde{S}_{j-1}\right) \mid F_{j-1}\right)\right]=0
\end{aligned}
$$

So, $\sum_{j=1}^{n} E Y_{j}^{2}=\operatorname{var}\left(\tilde{S}_{n}\right) \geq \sum_{k=1}^{n} E\left(\tilde{S}_{k}^{2} I_{A_{k}}\right) \geq \varepsilon^{2} \sum_{k=1}^{n} E I_{A_{k}}=\varepsilon^{2} P(A)$.

Therefore, we have $P\left(\max _{1 \leq k \leq n}\left|\tilde{S}_{k}\right| \geq \varepsilon\right) \leq \frac{\sum_{j=1}^{n} E Y_{j}^{2}}{\varepsilon^{2}}$.

Second, we want to show $\sum_{j=1}^{n} Y_{j}<\infty$ almost surely.

For $\forall \varepsilon>0$,

$$
\begin{aligned}
& P\left(\bigcup_{k=1}^{\infty}\left\{\left|\tilde{S}_{n+k}-\tilde{S}_{n}\right|>\varepsilon\right\}\right)=P\left(\lim _{m \rightarrow \infty} \bigcup_{k=1}^{m}\left\{\left|\tilde{S}_{n+k}-\tilde{S}_{n}\right|>\varepsilon\right\}\right) \\
= & \lim _{m \rightarrow \infty} P\left(\bigcup_{k=1}^{m}\left\{\left|\tilde{S}_{n+k}-\tilde{S}_{n}\right|>\varepsilon\right\}\right)=\lim _{m \rightarrow \infty} P\left(\left\{\max _{1 \leq k \leq m}\left|\tilde{S}_{n+k}-\tilde{S}_{n}\right|>\varepsilon\right\}\right) \\
\leq & \frac{\lim _{m \rightarrow \infty} \sum_{k=n+1}^{n+m} E Y_{k}^{2}}{\varepsilon^{2}}=\frac{\sum_{k=n+1}^{\infty} E Y_{k}^{2}}{\varepsilon^{2}} \rightarrow 0 \text { almost surely, }
\end{aligned}
$$


as $n \rightarrow \infty$. Since $E X_{i}^{2}$ is bounded, there exists $M$ such that $E X_{i}^{2} \leq M$. So,

$$
\sum_{k=n+1}^{\infty} E Y_{k}^{2} \leq M \sum_{k=n+1}^{\infty} \frac{1}{k^{2}}<\infty
$$

Therefore, $\tilde{S}_{n}$ is a Cauchy sequence. $\tilde{S}_{n}$ converges almost surely.

Finally, by Kronecker's lemma (Shiryaev, 1984 Lemma IV.3.2), we have

$$
\frac{S_{n}}{n}=\frac{\sum_{j=1}^{n} X_{j}}{n}=\frac{\sum_{j=1}^{j} n Y_{j}}{n} \rightarrow 0 \text { almost surely. }
$$

\subsection{The Establish of Maximum Likelihood Esti- mation}

When the toxicity response is homogeneous, the maximum likelihood estimation does not exist. The maximum likelihood estimation may exist when we have observed some kind of heterogeneity in the toxicity response. The fundamental heterogeneity is that a toxicity is observed after a series of non-toxicity or a non-toxicity is observed after a series of toxicities. Define

$$
n_{0}=\inf \left\{n: 0<\sum_{j=1}^{n} y_{j}<n\right\}
$$

where $n_{0}$ is the first time that both outcomes of the response are observed.

Theorem 1. If condition 2 holds, $P\left(n_{0}<\infty\right)=1$. With probability 1 , heterogeneity can be achieved after finite number of patients.

Proof. Define $T_{n}=\sum_{j=1}^{n}\left\{y_{j}-R\left(x_{j}, z_{j}\right)\right\}$. According to the definition, $T_{n}$ is a martingale. The limit theorem for martingale (Shiryayev, 1984, Section 7.3, Corollary 
2) shows that $\frac{T_{n}}{n}$ tends to zero almost surely. Furthermore, condition 2 leads to

$$
0<m_{R} \leq \frac{1}{n} \sum_{j=1}^{n} R\left(x_{j}, z_{j}\right) \leq M_{R}<1
$$

which yields that for large enough $n, 1 \leq \sum_{j=1}^{n} y_{j} \leq n-1$ almost surely. It then follows that $P\left(n_{0}<\infty\right)=1$.

Corollary 2. Heterogeneity with some other definitions such as the first time that two toxicities are observed or two non-toxicity are observed:

$$
n_{1}=\inf \left\{n: 2 \leq \sum_{j=1}^{n} y_{j} \leq n-2\right\}
$$

can be achieved after finite number of patients.

The maximum likelihood estimation does exist when we have observed some kind of heterogeneity in the toxicity response, such as for a subset of the domain of the biomarker $z$, a toxicity is observed after a series of non-toxicity or a non-toxicity is observed after a series of toxicities. Define

$$
n_{2}=\inf \left\{n: 0<\sum_{j=1}^{n} y_{j} I\left\{z_{j} \in A\right\}<\sum_{j=1}^{n} I\left\{z_{j} \in A\right\}\right\}
$$

where $A$ is the subset of the domain of the biomarker $z$ and $P(z \in A)>0 . n_{2}$ is the first time that for patients with biomarker in the subset, both outcomes of the response are observed and the MLE can be calculated.

Corollary 3. If condition 2 holds, $P\left(n_{2}<\infty\right)=1$. With probability 1, for the defined subset of biomarker, heterogeneity can be achieved after finite number of patients. 
Proof. It is obvious that $\sum_{j=1}^{n} I\left\{z_{j} \in A\right\}=2$ can be achieved after finite number of patients. Define $T_{n}=\sum_{j=1}^{n}\left\{y_{j}-R\left(x_{j}, z_{j}\right)\right\} I\left\{z_{j} \in A\right\}$. According to the definition, $T_{n}$ is a martingale. The limit theorem for martingale (Shiryayev, 1984) shows that $\frac{T_{n}}{n}$ tends to zero almost surely. Furthermore, condition 2 leads to

$0<m_{R} \sum_{j=1}^{n} I\left\{z_{j} \in A\right\} \leq \frac{1}{n} \sum_{j=1}^{n} R\left(x_{j}, z_{j}\right) I\left\{z_{j} \in A\right\} \leq M_{R} \sum_{j=1}^{n} I\left\{z_{j} \in A\right\}<\sum_{j=1}^{n} I\left\{z_{j} \in A\right\}$

which yields that for large enough $n, 0<\sum_{j=1}^{n} y_{j} I\left\{z_{j} \in A\right\}<\sum_{j=1}^{n} I\left\{z_{j} \in A\right\}$ almost surely. It then follows that $P\left(n_{2}<\infty\right)=1$.

Theorem 2. (The existence of MLE) For the working model $\psi\left(d_{i}, z, a, b\right)=$ $\alpha_{i}^{\exp (a+b z)}$, the maximum likelihood estimates for the parameters $a$ and $b$ are finite when we have observed some kind of heterogeneity in the toxicity response.

Proof. For fixed $n$, suppose $\hat{a}$ and $\hat{b}$ are the MLE on $[-\infty,+\infty] \times[-\infty,+\infty]$, where $\hat{a}$ and $\hat{b}$ could be infinity. We want to show $\hat{a}$ and $\hat{b}$ are not infinity. Consider the first order derivatives of the log-likelihood:

$$
\left\{\begin{aligned}
\frac{\partial l_{n}(a, b)}{\partial a} & =\sum_{j=1}^{n}\left[y_{j} \frac{\frac{\partial \psi}{\partial a}}{\psi}\left(x_{j}, z_{j}, a, b\right)+\left(1-y_{j}\right) \frac{-\frac{\partial \psi}{\partial a}}{1-\psi}\left(x_{j}, z_{j}, a, b\right)\right] \\
\frac{\partial l_{n}(a, b)}{\partial b} & =\sum_{j=1}^{n}\left[y_{j} \frac{\frac{\partial \psi}{\partial b}}{\psi}\left(x_{j}, z_{j}, a, b\right)+\left(1-y_{j}\right) \frac{-\frac{\partial \psi}{\partial b}}{1-\psi}\left(x_{j}, z_{j}, a, b\right)\right]
\end{aligned}\right.
$$


For the first order derivatives of the working model $\psi(x, z, a, b)$, we have

$$
\begin{aligned}
\frac{\frac{\partial \psi}{\partial a}}{\psi}(x, z, a, b) & =\log \tilde{\alpha}_{i} \exp (a+b z) \rightarrow\left\{\begin{array}{c}
-\infty \text { when } a+b z \rightarrow+\infty \\
0^{-} \text {when } a+b z \rightarrow-\infty
\end{array}\right. \\
\frac{-\frac{\partial \psi}{\partial a}}{1-\psi}(x, z, a, b) & =\frac{-\tilde{\alpha}_{i}^{\exp (a+b z)} \log \tilde{\alpha}_{i} \exp (a+b z)}{1-\tilde{\alpha}_{i}^{\exp (a+b z)}} \\
& \rightarrow\left\{\begin{array}{r}
0^{+} \text {when } a+b z \rightarrow+\infty \\
1+\log \tilde{\alpha}_{i} \exp (a+b z) \rightarrow 1^{-} \text {when } a+b z \rightarrow-\infty
\end{array}\right.
\end{aligned}
$$

where $x$ is the dose and $\tilde{\alpha}_{i}$ is the skeleton for the dose $x$.

We want to show $\hat{a}$ and $\hat{b}$ are finite, we just need to show it is impossible that either $\hat{a}$ or $\hat{b}$ is infinity.

(1) If $\hat{b}$ is finite, consider $a \rightarrow+\infty$ and we have

$$
\frac{\partial l_{n}(a, b)}{\partial a} \rightarrow-\infty
$$

almost surely. Thus, $l_{n}(a, b)$ is decreasing in $a$, when $b$ is finite and fixed and $a \rightarrow+\infty$. So $\hat{b}$ is finite and $\hat{a}=+\infty$ won't be the MLE.

(2) If $\hat{b}$ is finite, consider $a \rightarrow-\infty$ and we have

$$
\frac{\partial l_{n}(a, b)}{\partial a}>0
$$

almost surely. Thus, $l_{n}(a, b)$ is increasing in $a$, when $b$ is finite and fixed and $a \rightarrow$ $-\infty$. So $\hat{b}$ is finite and $\hat{a}=-\infty$ won't be the MLE. 
Then consider $\hat{b}$ is infinity and we have

$$
\begin{aligned}
\frac{\partial l_{n}(a, b)}{\partial a} & =\sum_{\left\{j: a+b z_{j}=+\infty\right\}} y_{j}(-\infty)+\sum_{\left\{j: a+b z_{j}=+\infty\right\}}\left(1-y_{j}\right) 0^{+} \\
& +\sum_{\left\{j: a+b z_{j}=-\infty\right\}} y_{j} \times 0^{-}+\sum_{\left\{j: a+b z_{j}=-\infty\right\}}\left(1-y_{j}\right) \times 1^{-}
\end{aligned}
$$

(3) If $\hat{b}=+\infty$ and $\hat{a}$ is finite, $\frac{\partial l_{n}(a, b)}{\partial a}$ has to be zero. Thus,

for $z_{j}>0$, i.e. $a+b z_{j}=+\infty, y_{j}$ must be 0 .

for $z_{j}<0$, i.e. $a+b z_{j}=-\infty, y_{j}$ must be 1 .

Both of them contradict with the heterogeneity in the toxicity response for the defined subsets of biomarker.

(4) If $\hat{b}=+\infty$ and $a \rightarrow+\infty$

for $z_{j}>-1$, i.e. $a+b z_{j}=+\infty, y_{j}$ must be 0 . Otherwise, $l_{n}(a, b)$ is decreasing in $a$, which indicates $\hat{b}=+\infty$ and $\hat{a}=+\infty$ won't be the MLE.

If $z_{j}<-1$ for all $j$, i.e. $a+b z_{j}=-\infty$, consider

$$
\frac{\partial l_{n}(a, b)}{\partial b}=\sum_{\left\{j: a+b z_{j}=-\infty\right\}} y_{j} \times 0^{-} z_{j}+\sum_{\left\{j: a+b z_{j}=-\infty\right\}}\left(1-y_{j}\right) \times 1^{-} z_{j}
$$

$l_{n}(a, b)$ is decreasing in $b$, which indicates $\hat{b}=+\infty$ and $\hat{a}=+\infty$ won't be the MLE.

(5)If $\hat{b}=+\infty$ and $a \rightarrow-\infty$

If $z_{j}>1$ for all $j$, i.e. $a+b z_{j}=+\infty$, consider

$$
\frac{\partial l_{n}(a, b)}{\partial b}=\sum_{\left\{j: a+b z_{j}=+\infty\right\}} y_{j}(-\infty) z_{j}+\sum_{\left\{j: a+b z_{j}=+\infty\right\}}\left(1-y_{j}\right) 0^{+} z_{j}
$$

$l_{n}(a, b)$ is decreasing in $b$, which indicates $\hat{b}=+\infty$ and $\hat{a}=-\infty$ won't be the MLE. for $z_{j}<1$, i.e. $a+b z_{j}=-\infty, y_{j}$ must be 1 . Otherwise, $l_{n}(a, b)$ is increasing in 
$a$, which indicates $\hat{b}=+\infty$ and $\hat{a}=-\infty$ won't be the MLE.

Similar arguments apply for $\hat{b}=-\infty$. Therefore, we prove the existence of MLE.

REMARK 1. Under very weak conditions, the existence of MLE can be shown for some other working models.

Theorem 3. (The uniqueness of MLE) If there exist $a_{0}$ and $b_{0}$ such that $\psi\left(d_{i}, z, a_{0}, b_{0}\right)=R\left(d_{i}, z\right)$ for $i=1, \ldots, k$ and $\forall z \in Z$, and $\psi(x, z, a, b)$ can be written as a function of $x$ and $a+b z$, i.e. $\psi(x, z, a, b)=\tilde{\psi}(x, a+b z)$, there exist $N$ such that for $n>N$, the maximum likelihood estimation of the parameter $a$ and $b$ is unique when the MLE exists.

Proof. Consider the first order derivatives and second order derivatives of the function $I_{n}$ :

$$
\begin{aligned}
\frac{\partial I_{n}(a, b)}{\partial a}= & \frac{1}{n} \sum_{j=1}^{n}\left[y_{j} \frac{\frac{\partial \psi}{\partial a}}{\psi}\left(x_{j}, z_{j}, a, b\right)+\left(1-y_{j}\right) \frac{-\frac{\partial \psi}{\partial a}}{1-\psi}\left(x_{j}, z_{j}, a, b\right)\right] \\
\frac{\partial I_{n}(a, b)}{\partial b}= & \frac{1}{n} \sum_{j=1}^{n}\left[y_{j} \frac{\frac{\partial \psi}{\partial b}}{\psi}\left(x_{j}, z_{j}, a, b\right)+\left(1-y_{j}\right) \frac{-\frac{\partial \psi}{\partial b}}{1-\psi}\left(x_{j}, z_{j}, a, b\right)\right] \\
\frac{\partial^{2} I_{n}(a, b)}{\partial a^{2}}= & \frac{1}{n} \sum_{j=1}^{n}\left[y_{j} \frac{\frac{\partial^{2} \psi}{\partial a^{2}} \psi-\left(\frac{\partial \psi}{\partial a}\right)^{2}}{\psi^{2}}\left(x_{j}, z_{j}, a, b\right)\right. \\
\frac{\partial^{2} I_{n}(a, b)}{\partial a \partial b}= & \left.\frac{\partial^{2} I_{n}(a, b)}{\partial b \partial a}\left(1-y_{j}\right) \frac{\frac{\partial^{2} \psi}{\partial a^{2}}(1-\psi)+\left(\frac{\partial \psi}{\partial a}\right)^{2}}{(1-\psi)^{2}}\left(x_{j}, z_{j}, a, b\right)\right] \\
= & \frac{1}{n} \sum_{j=1}^{n}\left[y_{j} \frac{\frac{\partial^{2} \psi}{\partial a \partial b} \psi-\left(\frac{\partial \psi}{\partial a}\right)\left(\frac{\partial \psi}{\partial b}\right)}{\psi^{2}}\left(x_{j}, z_{j}, a, b\right)\right. \\
& \left.-\left(1-y_{j}\right) \frac{\frac{\partial^{2} \psi}{\partial a \partial b}(1-\psi)+\left(\frac{\partial \psi}{\partial a}\right)\left(\frac{\partial \psi}{\partial b}\right)}{(1-\psi)^{2}}\left(x_{j}, z_{j}, a, b\right)\right]
\end{aligned}
$$




$$
\begin{aligned}
\frac{\partial^{2} I_{n}(a, b)}{\partial b^{2}}= & \frac{1}{n} \sum_{j=1}^{n}\left[y_{j} \frac{\frac{\partial^{2} \psi}{\partial b^{2}} \psi-\left(\frac{\partial \psi}{\partial b}\right)^{2}}{\psi^{2}}\left(x_{j}, z_{j}, a, b\right)\right. \\
& \left.-\left(1-y_{j}\right) \frac{\frac{\partial^{2} \psi}{\partial b^{2}}(1-\psi)+\left(\frac{\partial \psi}{\partial b}\right)^{2}}{(1-\psi)^{2}}\left(x_{j}, z_{j}, a, b\right)\right]
\end{aligned}
$$

$\frac{\partial^{2} I_{n}(a, b)}{\partial a^{2}}$ can be rewritten as

$$
\frac{\partial^{2} I_{n}(a, b)}{\partial a^{2}}=\frac{1}{n} \sum_{j=1}^{n}\left[\left\{\frac{y_{j}}{\psi}-\frac{1-y_{j}}{1-\psi}\right\} \frac{\partial^{2} \psi}{\partial a^{2}}\right]-\frac{1}{n} \sum_{j=1}^{n}\left[\left\{\frac{y_{j}}{\psi^{2}}+\frac{1-y_{j}}{(1-\psi)^{2}}\right\}\left(\frac{\partial \psi}{\partial a}\right)^{2}\right]
$$

The first term is a zero mean martingale, by lemma 1, it converges to 0 almost surely. Therefore,

$$
\frac{\partial^{2} I_{n}(a, b)}{\partial a^{2}}+\frac{1}{n} \sum_{j=1}^{n}\left[\left\{\frac{y_{j}}{\psi^{2}}+\frac{1-y_{j}}{(1-\psi)^{2}}\right\}\left(\frac{\partial \psi}{\partial a}\right)^{2}\right] \rightarrow 0 \text { almost surely. }
$$

So,

$$
\frac{\partial^{2} I_{n}(a, b)}{\partial a^{2}}<0 \text { almost surely }
$$

We can prove similar results:

$$
\begin{aligned}
& \frac{\partial^{2} I_{n}(a, b)}{\partial a \partial b}<0 \text { almost surely, } \\
& \frac{\partial^{2} I_{n}(a, b)}{\partial b^{2}}<0 \text { almost surely. }
\end{aligned}
$$

Since $\psi\left(d_{i}, z, a, b\right)$ can be written as a function of $a+b z$, we have $\psi\left(d_{i}, z, a, b\right)=\psi_{i}(a+$ $b z)$. We have $\frac{\partial \psi\left(d_{i}, z, a, b\right)}{\partial a}=\psi_{i}^{\prime}, \frac{\partial \psi\left(d_{i}, z, a, b\right)}{\partial b}=\psi_{i}^{\prime} z, \frac{\partial^{2} \psi\left(d_{i}, z, a, b\right)}{\partial a^{2}}=\psi_{i}^{\prime \prime}, \frac{\partial^{2} \psi\left(d_{i}, z, a, b\right)}{\partial a \partial b}=\psi_{i}^{\prime \prime} z$ and $\frac{\partial^{2} \psi\left(d_{i}, z, a, b\right)}{\partial b^{2}}=\psi_{i}^{\prime \prime} z^{2}$. 
Consider the Hessian matrix

$$
\left|\begin{array}{cc}
\frac{\partial^{2} I_{n}}{\partial a^{2}} & \frac{\partial^{2} I_{n}}{\partial a \partial b} \\
\frac{\partial^{2} I_{n}}{\partial b \partial a} & \frac{\partial^{2} I_{n}}{\partial b^{2}}
\end{array}\right|=\frac{\partial^{2} I_{n}}{\partial a^{2}} \frac{\partial^{2} I_{n}}{\partial b^{2}}-\left(\frac{\partial^{2} I_{n}}{\partial a \partial b}\right)^{2}
$$

By Cauchy-Schwarz inequality, the Hessian matrix is non-negative almost surely. Further, since biomarker $z$ is not placed on a single point, the equality does not hold in the long run and the Hessian matrix is positive almost surely. Therefore, the MLE is unique when it exists.

\subsection{Convergence}

Theorem 4. If there exist $a_{0}$ and $b_{0}$ such that $\psi\left(d_{i}, z, a_{0}, b_{0}\right)=R\left(d_{i}, z\right)$ for $i=$ $1, \ldots, k$ and $\forall z \in Z, \hat{a}_{n}$ and $\hat{b}_{n}$ are the maximum likelihood estimation of the parameter $a$ and $b$ in the working model $\psi\left(d_{i}, z, a, b\right)$, we have

$$
\begin{aligned}
& \hat{a}_{n} \rightarrow a_{0} \quad \text { almost surely } \\
& \hat{b}_{n} \rightarrow b_{0} \quad \text { almost surely, }
\end{aligned}
$$

and if $d(z)$ is the target dose for the value $z$, for the recommended dose $x_{n+1}(z)$ given the value $z$, we have

$$
x_{n+1}(z) \rightarrow d(z) \text { almost surely }
$$

Proof. We show the proof by three steps. First we show $\tilde{I}_{n}(a, b)$ is uniquely maximized at $\left(a_{0}, b_{0}\right)$. Second we show $\sup _{\substack{a \in\left[m_{a}, M_{a}\right] \\ b \in\left[m_{b}, M_{b}\right]}}\left|I_{n}(a, b)-\tilde{I}_{n}(a, b)\right| \rightarrow 0$ almost surely. Third, show the result $\hat{a}_{n} \rightarrow a_{0}$ and $\hat{b}_{n} \rightarrow b_{0}$ almost surely will follow. 
First, for fixed $t$, the function $f_{1}(x)=t \ln x+(1-t) \ln (1-x)$ reaches its $\mathrm{u}$ nique maximum at $x=t$. Then each term in the summation in $\tilde{I}_{n}(a, b)$ reaches its maximum at $\psi\left(x_{j}, z_{j}, a, b\right)=R\left(x_{j}, z_{j}\right)$. Since there exist $a_{0}$ and $b_{0}$ such that $\psi\left(d_{i}, z, a_{0}, b_{0}\right)=R\left(d_{i}, z\right), \tilde{l}_{n}(a, b)$ is maximized at $\left(a_{0}, b_{0}\right)$. Suppose there exist $a_{1}$ and $b_{1}$ such that $\tilde{l}_{n}(a, b)$ reaches its maximum, then $\psi\left(x_{j}, z_{j}, a_{1}, b_{1}\right)=R\left(x_{j}, z_{j}\right)=$ $\psi\left(x_{j}, z_{j}, a_{0}, b_{0}\right)$. It follows that $a_{1}+b_{1} z_{j}=a_{0}+b_{0} z_{j}$ for $1 \leq j \leq n$. Since the distribution of $z$ is not placed on a single point, $a=a_{0}$ and $b=b_{0}$. Therefore, $\tilde{I}_{n}(a, b)$ is uniquely maximized at $\left(a_{0}, b_{0}\right)$.

Second, consider

$$
S_{n}(a, b)=I_{n}(a, b)-\tilde{I}_{n}(a, b)=\frac{1}{n} \sum_{j=1}^{n}\left\{y_{j}-R\left(x_{j}, z_{j}\right)\right\} \log \frac{\psi\left(x_{j}, z_{j}, a, b\right)}{1-\psi\left(x_{j}, z_{j}, a, b\right)}
$$

Obviously, $S_{n}(a, b)$ is a zero-mean martingale. Since $a \in\left[m_{a}, M_{a}\right], b \in\left[m_{b}, M_{b}\right]$ and $z$ is bounded, there exists $0<m_{\psi}<M_{\psi}<1$ such that $0<m_{\psi}<\psi\left(x_{j}, z_{j}, a, b\right)<$ $M_{\psi}<1$. Thus, $\log \frac{\psi\left(x_{j}, z_{j}, a, b\right)}{1-\psi\left(x_{j}, z_{j}, a, b\right)}$ is bounded uniformly on $\left[m_{a}, M_{a}\right] \times\left[m_{b}, M_{b}\right]$. Since $-1 \leq y_{j}-R\left(x_{j}, z_{j}\right) \leq 1$, we have $E\left[\left\{y_{j}-R\left(x_{j}, z_{j}\right)\right\} \log \frac{\psi\left(x_{j}, z_{j}, a, b\right)}{1-\psi\left(x_{j}, z_{j}, a, b\right)}\right]^{2}$ is uniformly bounded. By lemma $1, I_{n}(a, b)-\tilde{I}_{n}(a, b) \rightarrow 0$ almost surely for $a \in\left[m_{a}, M_{a}\right]$, $b \in\left[m_{b}, M_{b}\right]$.

Since $S_{n}(a, b) \rightarrow 0$ almost surely, there exists $\Omega_{1} \subseteq\left[m_{a}, M_{a}\right] \times\left[m_{b}, M_{b}\right]$ and $P\left(\Omega_{1}\right)=1$, for any $(a, b) \in \Omega_{1}$ we have $S_{n}(a, b) \rightarrow 0$. Suppose $\sup _{(a, b) \in \Omega_{1}}\left|S_{n}(a, b)\right| \not \rightarrow 0$ almost surely, then there exists $\varepsilon>0$ and sequence $\left\{n_{k}\right\}$ and $\left\{a_{n_{k}}\right\}$ and $\left\{b_{n_{k}}\right\}$ such that $\left|S_{n_{k}}\left(a_{n_{k}}, b_{n_{k}}\right)\right| \geq \varepsilon$. With $\left\{a_{n_{k}}\right\}$ and $\left\{b_{n_{k}}\right\}$ bounded, there exists a subsequence $\left\{n_{k_{l}}\right\}$ such that $a_{n_{k_{l}}} \rightarrow a^{*}$ and $b_{n_{k_{l}}} \rightarrow b^{*}$. Without loss of generality, suppose $a_{n_{k}} \rightarrow a^{*}$ and $b_{n_{k}} \rightarrow b^{*}$. Since $P\left(\left(a^{*}, b^{*}\right) \notin \Omega_{1}\right)=0$, we only need to consider 
$\left(a^{*}, b^{*}\right) \in \Omega_{1} . S_{n_{k}}\left(a_{n_{k}}, b_{n_{k}}\right)$ can be rewritten as

$$
S_{n_{k}}\left(a_{n_{k}}, b_{n_{k}}\right)=S_{n_{k}}\left(a_{n_{k}}, b_{n_{k}}\right)-S_{n_{k}}\left(a^{*}, b^{*}\right)+S_{n_{k}}\left(a^{*}, b^{*}\right) .
$$

Then

$$
\begin{aligned}
S_{n_{k}}\left(a_{n_{k}}, b_{n_{k}}\right)-S_{n_{k}}\left(a^{*}, b^{*}\right)=\frac{1}{n_{k}} \sum_{j=1}^{n_{k}}\left\{y_{j}-R\left(x_{j}, z_{j}\right)\right\} & \left\{\log \frac{\psi\left(x_{j}, z_{j}, a_{n_{k}}, b_{n_{k}}\right)}{1-\psi\left(x_{j}, z_{j}, a_{n_{k}}, b_{n_{k}}\right)}\right. \\
& \left.-\log \frac{\psi\left(x_{j}, z_{j}, a^{*}, b^{*}\right)}{1-\psi\left(x_{j}, z_{j}, a^{*}, b^{*}\right)}\right\}
\end{aligned}
$$

By the continuity of $\psi(\cdot)$ in proposition 2, we have $\left|S_{n_{k}}\left(a_{n_{k}}, b_{n_{k}}\right)-S_{n_{k}}\left(a^{*}, b^{*}\right)\right| \rightarrow 0$ almost surely as $n_{k} \rightarrow \infty$. $S_{n_{k}}\left(a^{*}, b^{*}\right) \rightarrow 0$ almost surely as $n_{k} \rightarrow \infty$ for $\left(a^{*}, b^{*}\right) \in \Omega_{1}$. Then the left side of equation (3.7) is large than $\varepsilon>0$ while the right side of the equation goes to 0 as $n_{k} \rightarrow \infty$. Therefore, $\sup _{\substack{a \in\left[m_{a}, M_{a}\right] \\ b \in\left[m_{b}, M_{b}\right]}}\left|S_{n}(a, b)\right| \rightarrow 0$ almost surely. Third, the convergence results $\sup _{\substack{a \in\left[m_{a}, M_{a}\right] \\ b \in\left[m_{b}, M_{b}\right]}}\left|S_{n}(a, b)\right| \rightarrow 0$ almost surely implies:

$$
\sup _{\substack{a \in\left[m_{a}, M_{a}\right] \\ b \in\left[m_{b}, M_{b}\right]}}\left|\left\{I_{n}(a, b)-I_{n}\left(a_{0}, b_{0}\right)\right\}-\left\{\tilde{I}_{n}(a, b)-\tilde{I}_{n}\left(a_{0}, b_{0}\right)\right\}\right| \rightarrow 0 \text { almost surely. }
$$

Let $\rho \geq 0$ and define the compact set $\Omega_{2}=\left\{(a, b):\left\|(a, b)-\left(a_{0}, b_{0}\right)\right\| \geq \rho\right\}$, where $\|\cdot\|$ is the Euclidean norm in $\mathbf{R}^{2}$. By the continuity of $\psi(\cdot)$ in proposition 2, $\tilde{I}_{n}(a, b)-\tilde{I}_{n}\left(a_{0}, b_{0}\right)$ is uniformly continuous. So it achieve its maximum on $\Omega_{2}$ denoted by $\delta=\sup _{(a, b) \in \Omega_{2}}\left\{\tilde{l}_{n}(a, b)-\tilde{l}_{n}\left(a_{0}, b_{0}\right)\right\}$. Since $\tilde{I}_{n}(a, b)$ is uniquely maximized at $\left(a_{0}, b_{0}\right)$, we have $\delta<0$. Since $\sup _{\substack{a \in\left[m_{a}, M_{a}\right] \\ b \in\left[m_{b}, M_{b}\right]}}\left|\left\{l_{n}(a, b)-l_{n}\left(a_{0}, b_{0}\right)\right\}-\left\{\tilde{l}_{n}(a, b)-\tilde{I}_{n}\left(a_{0}, b_{0}\right)\right\}\right| \rightarrow 0$ almost surely, there exists $N_{\rho}$ such that for $n>N_{\rho}, \sup _{(a, b) \in \Omega_{2}}\left\{I_{n}(a, b)-I_{n}\left(a_{0}, b_{0}\right)\right\}<$ $\frac{1}{2} \delta<0$ almost surely. On the other hand, $I_{n}\left(a_{0}, b_{0}\right)-I_{n}\left(a_{0}, b_{0}\right)=0$, so we have 
$I_{n}\left(\hat{a}_{n}, \hat{b}_{n}\right)-I_{n}\left(a_{0}, b_{0}\right) \geq 0$ which shows that the MLE is not in $\Omega_{2}$. Since $\rho$ is arbitrarily chosen, the result $\hat{a}_{n} \rightarrow a_{0}$ and $\hat{b}_{n} \rightarrow b_{0}$ almost surely follows.

Subsequently, $\psi\left(d_{i}, z, \hat{a}_{n}, \hat{b}_{n}\right) \rightarrow \psi\left(d_{i}, z, a_{0}, b_{0}\right)=R\left(d_{i}, z\right)$ almost surely. Therefore, the recommended dose $x_{n+1}(z)$ given $z$ converges to the target dose $d(z)$ almost surely.

Condition 8. For $a \in\left[m_{a}, M_{a}\right], b \in\left[m_{b}, M_{b}\right]$ and $z \in\left[m_{z}, M_{z}\right]$,

$$
\frac{\partial^{2} \psi}{\partial a^{2}} \psi-\left(\frac{\partial \psi}{\partial a}\right)^{2} \leq 0
$$

and

$$
-\frac{\partial^{2} \psi}{\partial a^{2}}(1-\psi)-\left(\frac{\partial \psi}{\partial a}\right)^{2} \leq 0
$$

with at least one inequality being strict.

Condition 8 is an additional assumption required for the consistency of the biomarker design using posterior mean. It is not required for the maximum likelihood estimator.

Theorem 5. If condition 8 holds and there exist $a_{0}$ and $b_{0}$ such that $\psi\left(d_{i}, z, a_{0}, b_{0}\right)=$ $R\left(d_{i}, z\right)$ for $i=1, \ldots, k$ and $\forall z \in Z, \psi(x, z, a, b)$ can be written as a function of $x$ and $a+b z$, i.e. $\psi(x, z, a, b)=\tilde{\psi}(x, a+b z), \tilde{a}_{n}$ and $\tilde{b}_{n}$ are the posterior mean of the parameter $a$ and $b$ in the working model $\psi\left(d_{i}, z, a, b\right)$, we have

$$
\begin{aligned}
& \tilde{a}_{n} \rightarrow a_{0} \quad \text { almost surely } \\
& \tilde{b}_{n} \rightarrow b_{0} \quad \text { almost surely, }
\end{aligned}
$$

and if $d(z)$ is the target dose for the value $z$, for the recommended dose $x_{n+1}(z)$ 
given the value $z$, we have

$$
x_{n+1}(z) \rightarrow d(z) \text { almost surely }
$$

Proof. Denote $\beta=(a, b)$. Consider the Taylor expansion at $\hat{\beta}_{n}=\left(\hat{a}_{n}, \hat{b}_{n}\right)$, since $\frac{\partial l_{n}\left(\hat{\beta}_{n}\right)}{\partial a}=0$ and $\frac{\partial l_{n}\left(\hat{\beta}_{n}\right)}{\partial b}=0$, we have

$$
l_{n}(\beta)=l_{n}\left(\hat{\beta}_{n}\right)+\frac{1}{2} D^{2} l_{n}\left(\beta^{*}\right)\left(\beta-\hat{\beta}_{n}\right)^{2}
$$

for some $\beta^{*}$ between $\beta$ and $\hat{\beta}_{n}$.

$$
\begin{aligned}
& D^{2} l_{n}\left(\beta^{*}\right)\left(\beta-\hat{\beta}_{n}\right) \\
= & \frac{\partial^{2} l_{n}\left(a^{*}, b^{*}\right)}{\partial a^{2}}\left(a-\hat{a}_{n}\right)^{2}+2 \frac{\partial^{2} l_{n}\left(a^{*}, b^{*}\right)}{\partial a \partial b}\left(a-\hat{a}_{n}\right)\left(b-\hat{b}_{n}\right)+\frac{\partial^{2} l_{n}\left(a^{*}, b^{*}\right)}{\partial b^{2}}\left(b-\hat{b}_{n}\right)^{2},
\end{aligned}
$$

where

$$
\begin{aligned}
\frac{\partial^{2} l_{n}}{\partial a^{2}} & =\sum_{j=1}^{n}\left[y_{j} \frac{\frac{\partial^{2} \psi}{\partial a^{2}} \psi-\left(\frac{\partial \psi}{\partial a}\right)^{2}}{\psi^{2}}-\left(1-y_{j}\right) \frac{\frac{\partial^{2} \psi}{\partial a^{2}}(1-\psi)+\left(\frac{\partial \psi}{\partial a}\right)^{2}}{(1-\psi)^{2}}\right] \\
\frac{\partial^{2} l_{n}}{\partial a \partial b} & =\sum_{j=1}^{n}\left[y_{j} z_{j} \frac{\frac{\partial^{2} \psi}{\partial a^{2}} \psi-\left(\frac{\partial \psi}{\partial a}\right)^{2}}{\psi^{2}}-\left(1-y_{j}\right) z_{j} \frac{\frac{\partial^{2} \psi}{\partial a^{2}}(1-\psi)+\left(\frac{\partial \psi}{\partial a}\right)^{2}}{(1-\psi)^{2}}\right] \\
\frac{\partial^{2} l_{n}}{\partial b^{2}} & =\sum_{j=1}^{n}\left[y_{j} z_{j}^{2} \frac{\frac{\partial^{2} \psi}{\partial a^{2}} \psi-\left(\frac{\partial \psi}{\partial a}\right)^{2}}{\psi^{2}}-\left(1-y_{j}\right) z_{j}^{2} \frac{\frac{\partial^{2} \psi}{\partial a^{2}}(1-\psi)+\left(\frac{\partial \psi}{\partial a}\right)^{2}}{(1-\psi)^{2}}\right] .
\end{aligned}
$$

Under condition 8, we have

$$
\sup D^{2} l_{n}\left(\beta^{*}\right)\left(\beta-\hat{\beta}_{n}\right)^{2} \rightarrow-\infty \text { as } n \rightarrow \infty
$$


Now, we express the posterior mean $\tilde{\beta}_{n}=\left(\tilde{a}_{n}, \tilde{b}_{n}\right)$ as

$$
\begin{aligned}
\tilde{a}_{n} & =\frac{\int_{-\infty}^{\infty} \int_{-\infty}^{\infty} a L_{n}(a, b) f(a) f(b) d a d b}{\int_{-\infty}^{\infty} \int_{-\infty}^{\infty} L_{n}(a, b) f(a) f(b) d a d b} \\
& =\hat{a}_{n}+\frac{\int_{-\infty}^{\infty} \int_{-\infty}^{\infty}\left(a-\hat{a}_{n}\right) L_{n}(a, b) f(a) f(b) d a d b}{\int_{-\infty}^{\infty} \int_{-\infty}^{\infty} L_{n}(a, b) f(a) f(b) d a d b}
\end{aligned}
$$

Furthermore,

$$
\begin{aligned}
& \left|\int_{-\infty}^{\infty} \int_{-\infty}^{\infty}\left(a-\hat{a}_{n}\right) L_{n}(\beta) f(a) f(b) d a d b\right| \\
= & \left|\int_{-\infty}^{\infty} \int_{-\infty}^{\infty}\left(a-\hat{a}_{n}\right) \exp \left\{l_{n}\left(\hat{\beta}_{n}\right)+\frac{1}{2} D^{2} l_{n}\left(\beta^{*}\right)\left(\beta-\hat{\beta}_{n}\right)^{2}\right\} f(a) f(b) d a d b\right| \\
\leq & L_{n}\left(\hat{\beta}_{n}\right) \int_{-\infty}^{\infty} \int_{-\infty}^{\infty}\left|a-\hat{a}_{n}\right| \exp \left\{\frac{1}{2} D^{2} l_{n}\left(\beta^{*}\right)\left(\beta-\hat{\beta}_{n}\right)^{2}\right\} f(a) f(b) d a d b .
\end{aligned}
$$

The above integral can be further broken into

$$
\iint_{\left\|\beta-\hat{\beta}_{n}\right\| \leq \varepsilon}\left|a-\hat{a}_{n}\right| \exp \left\{\frac{1}{2} D^{2} l_{n}\left(\beta^{*}\right)\left(\beta-\hat{\beta}_{n}\right)^{2}\right\} f(a) f(b) d a d b \leq \delta
$$

for a given $\delta>0$, and

$$
\iint_{\mid \beta-\hat{\beta}_{n} \|>\varepsilon}\left|a-\hat{a}_{n}\right| \exp \left\{\frac{1}{2} D^{2} l_{n}\left(\beta^{*}\right)\left(\beta-\hat{\beta}_{n}\right)^{2}\right\} f(a) f(b) d a d b \rightarrow 0
$$

as $n \rightarrow \infty$ by (3.8). Since we choose $\delta$ to be arbitrarily small independent of $n$, the second term in (3.9) is arbitrarily close to 0 as sample size increases, we have $\left|\tilde{a}_{n}-\hat{a}_{n}\right| \rightarrow 0$ almost surely. So we prove $\left(\tilde{a}_{n}, \tilde{b}_{n}\right) \rightarrow\left(a_{0}, b_{0}\right)$ almost surely. 


\subsection{Asymptotic normality}

Theorem 6. If there exist $a_{0}$ and $b_{0}$ such that $\psi\left(d_{i}, z, a_{0}, b_{0}\right)=R\left(d_{i}, z\right)$ for $i=$ $1, \ldots, k$ and $\forall z \in Z$, and $\hat{a}_{n}$ and $\hat{b}_{n}$ are the maximum likelihood estimation of the parameter $a$ and $b$ in the working model $\psi\left(d_{i}, z, a, b\right)$, we have the asymptotic normality:

$$
\sqrt{n}\left(\left(\begin{array}{c}
\hat{a}_{n} \\
\hat{b}_{n}
\end{array}\right)-\left(\begin{array}{c}
a_{0} \\
b_{0}
\end{array}\right)\right) \rightarrow N\left(0,\left(\begin{array}{cc}
E S_{1}^{2} & E S_{1} S_{2} \\
E S_{2} S_{1} & E S_{2}^{2}
\end{array}\right)^{-1}\right) \text { in distribution, }
$$

where

$$
\begin{aligned}
s(x, y, z, a, b) & =y \log \{\psi(x, z, a, b)\}+(1-y) \log \{1-\psi(x, z, a, b)\} \\
s_{1}(x, y, z, a, b) & =\frac{\partial s(x, y, z, a, b)}{\partial a}=y \frac{\frac{\partial \psi}{\partial a}}{\psi}-(1-y) \frac{\frac{\partial \psi}{\partial a}}{1-\psi} \\
s_{2}(x, y, z, a, b) & =\frac{\partial s(x, y, z, a, b)}{\partial b}=y \frac{\frac{\partial \psi}{\partial b}}{\psi}-(1-y) \frac{\frac{\partial \psi}{\partial b}}{1-\psi} \\
s_{11}(x, y, z, a, b) & =\frac{\partial^{2} s(x, y, z, a, b)}{\partial a^{2}}=y \frac{\frac{\partial^{2} \psi}{\partial a^{2}} \psi-\left(\frac{\partial \psi}{\partial a}\right)^{2}}{\psi^{2}}-(1-y) \frac{\frac{\partial^{2} \psi}{\partial a^{2}}(1-\psi)+\left(\frac{\partial \psi}{\partial a}\right)^{2}}{(1-\psi)^{2}} \\
s_{12}(x, y, z, a, b) & =s_{22}(x, y, z, a, b)=\frac{\partial^{2} s(x, y, z, a, b)}{\partial a \partial b}=\frac{\partial^{2} s(x, y, z, a, b)}{\partial b \partial a} \\
& =y \frac{\frac{\partial^{2} \psi}{\partial \partial \partial b} \psi-\left(\frac{\partial \psi}{\partial a}\right)\left(\frac{\partial \psi}{\partial b}\right)}{\psi^{2}}-(1-y) \frac{\frac{\partial^{2} \psi}{\partial a \partial b}(1-\psi)+\left(\frac{\partial \psi}{\partial a}\right)\left(\frac{\partial \psi}{\partial b}\right)}{(1-\psi)^{2}} \\
s_{22}(x, y, z, a, b) & =\frac{\partial^{2} s(x, y, z, a, b)}{\partial b^{2}}=y \frac{\frac{\partial^{2} \psi}{\partial b^{2}} \psi-\left(\frac{\partial \psi}{\partial b}\right)^{2}}{\psi^{2}}-(1-y) \frac{\frac{\partial^{2} \psi}{\partial b^{2}}(1-\psi)+\left(\frac{\partial \psi}{\partial b}\right)^{2}}{(1-\psi)^{2}} .
\end{aligned}
$$

Suppose $S_{1}=s_{1}\left(d(z), y, z, a_{0}, b_{0}\right)$ and $S_{2}=s_{2}\left(d(z), y, z, a_{0}, b_{0}\right)$, where $d(z)$ is the target dose for the value z, ES $S_{1}^{2}, E S_{1} S_{2}$ and $E S_{2}^{2}$ exist. 
Proof. A Taylor expansion of

$$
\left\{\begin{array}{l}
\frac{\partial l_{n}(a, b)}{\partial a}=0 \\
\frac{\partial l_{n}(a, b)}{\partial b}=0
\end{array}\right.
$$

around $\left(a_{0}, b_{0}\right)$ leads to

$$
-\left(\begin{array}{c}
\frac{1}{\sqrt{n}} \sum_{j=1}^{n} s_{1}\left(x_{j}, y_{j}, z_{j}, a_{0}, b_{0}\right) \\
\frac{1}{\sqrt{n}} \sum_{j=1}^{n} s_{2}\left(x_{j}, y_{j}, z_{j}, a_{0}, b_{0}\right)
\end{array}\right) \approx A \sqrt{n}\left(\begin{array}{c}
\hat{a}_{n}-a_{0} \\
\hat{b}_{n}-b_{0}
\end{array}\right)
$$

where

$$
A=\left(\begin{array}{ll}
\frac{1}{n} \sum_{j=1}^{n} s_{11}\left(x_{j}, y_{j}, z_{j}, a_{0}, b_{0}\right) & \frac{1}{n} \sum_{j=1}^{n} s_{12}\left(x_{j}, y_{j}, z_{j}, a_{0}, b_{0}\right) \\
\frac{1}{n} \sum_{j=1}^{n} s_{21}\left(x_{j}, y_{j}, z_{j}, a_{0}, b_{0}\right) & \frac{1}{n} \sum_{j=1}^{n} s_{22}\left(x_{j}, y_{j}, z_{j}, a_{0}, b_{0}\right)
\end{array}\right) .
$$

Accordingly to the definition, we have

$$
\begin{aligned}
\sum_{j=1}^{n} s_{1}^{2}\left(x_{j}, y_{j}, z_{j}, a_{0}, b_{0}\right) & =\sum_{j=1}^{n}\left[y_{j} \frac{\left(\frac{\partial \psi}{\partial a}\right)^{2}}{\psi^{2}}+\left(1-y_{j}\right) \frac{\left(\frac{\partial \psi}{\partial a}\right)^{2}}{(1-\psi)^{2}}\right] \\
\sum_{j=1}^{n} s_{1} s_{2}\left(x_{j}, y_{j}, z_{j}, a_{0}, b_{0}\right) & =\sum_{j=1}^{n} s_{2} s_{1}\left(x_{j}, y_{j}, z_{j}, a_{0}, b_{0}\right) \\
& =\sum_{j=1}^{n}\left[y_{j} \frac{\frac{\partial \psi}{\partial a} \frac{\partial \psi}{\partial b}}{\psi^{2}}+\left(1-y_{j}\right) \frac{\frac{\partial \psi}{\partial a} \frac{\partial \psi}{\partial b}}{(1-\psi)^{2}}\right] \\
\sum_{j=1}^{n} s_{2}^{2}\left(x_{j}, y_{j}, z_{j}, a_{0}, b_{0}\right) & =\sum_{j=1}^{n}\left[y_{j} \frac{\left(\frac{\partial \psi}{\partial b}\right)^{2}}{\psi^{2}}+\left(1-y_{j}\right) \frac{\left(\frac{\partial \psi}{\partial b}\right)^{2}}{(1-\psi)^{2}}\right] .
\end{aligned}
$$

According to the arguments in theorem 2, we have

$$
\frac{1}{n} \sum_{j=1}^{n} s_{11}\left(x_{j}, y_{j}, z_{j}, a_{0}, b_{0}\right)+\frac{1}{n} \sum_{j=1}^{n} s_{1}^{2}\left(x_{j}, y_{j}, z_{j}, a_{0}, b_{0}\right) \rightarrow 0 \text { almost surely. }
$$


Since $R\left(d_{i}, z\right)$ and $\psi(x, z, a, b)$ are monotonic in $z$, suppose they are decreasing in $z$, we may have a partition for $z:\left(-\infty, z_{1}^{*}\right),\left(z_{1}^{*}, z_{2}^{*}\right), \ldots,\left(z_{k-1}^{*},+\infty\right)$, such that the target dose for the $i t h$ interval is $d_{i}$ respectively.

For any small enough $\varepsilon>0$, there exist $N$ for $n>N$, the recommended dose for $z \in\left(-\infty, z_{1}^{*}-\varepsilon\right) \cup\left(z_{1}^{*}+\varepsilon, z_{2}^{*}-\varepsilon\right) \cup \cdots \cup\left(z_{k-1}^{*}+\varepsilon,+\infty\right), x_{n+1}(z)=d(z)$ almost surely. Thus, we have

$$
\frac{1}{n} \sum_{j=1}^{n} s_{1}^{2}\left(x_{j}, y_{j}, z_{j}, a_{0}, b_{0}\right) \rightarrow E S_{1}^{2} \text { almost surely. }
$$

Furthermore, we have

$$
\frac{1}{n} \sum_{j=1}^{n} s_{11}\left(x_{j}, y_{j}, z_{j}, a_{0}, b_{0}\right) \rightarrow-E S_{1}^{2} \text { almost surely. }
$$

Therefore, matrix $A \rightarrow-\left(\begin{array}{cc}E S_{1}^{2} & E S_{1} S_{2} \\ E S_{2} S_{1} & E S_{2}^{2}\end{array}\right)$ almost surely.

The central limit theorem implies that the asymptotic distribution of (3.9) is normal with zero mean vector and covariance matrix $B$ :

$$
\left(\begin{array}{c}
\frac{1}{\sqrt{n}} \sum_{j=1}^{n} s_{1}\left(x_{j}, y_{j}, z_{j}, a_{0}, b_{0}\right) \\
\frac{1}{\sqrt{n}} \sum_{j=1}^{n} s_{2}\left(x_{j}, y_{j}, z_{j}, a_{0}, b_{0}\right)
\end{array}\right) \rightarrow N(0, B) \text { in distribution, }
$$

where $B=\left(\begin{array}{cc}E S_{1}^{2} & E S_{1} S_{2} \\ E S_{2} S_{1} & E S_{2}^{2}\end{array}\right)$. 
Solving (3.9) yield

$$
\sqrt{n}\left(\left(\begin{array}{l}
\hat{a}_{n} \\
\hat{b}_{n}
\end{array}\right)-\left(\begin{array}{c}
a_{0} \\
b_{0}
\end{array}\right)\right)=-A^{-1}\left(\begin{array}{c}
\frac{1}{\sqrt{n}} \sum_{j=1}^{n} s_{1} \\
\frac{1}{\sqrt{n}} \sum_{j=1}^{n} s_{2}
\end{array}\right) .
$$

Therefore, the asymptotic covariance matrix is

$$
A^{-1} B\left(A^{-1}\right)^{\prime}=\left(\begin{array}{cc}
E S_{1}^{2} & E S_{1} S_{2} \\
E S_{2} S_{1} & E S_{2}^{2}
\end{array}\right)^{-1}
$$

Therefore, the theorem holds. 


\section{Chapter 4}

\section{Biomarker-based Design for}

\section{Multiple-agent Trial}

In single-agent trials, "treatment" is synonymous with "dose level". In multipleagent trials, we use "treatment" to denote combinations of agents. For single-agent trials, the ordering of the probability of toxicity is assumed monotonic with dose increment. For multiple-agent trials, assume monotonicity for each agent separately, but the combined ordering may not be fully known, resulting in a "partial order".

\subsection{Statistical Method}

\subsubsection{Toxicity Probability Model}

For multiple-agent trial, suppose the dose combinations follow a partial order in which there are $M$ possible simple orders consistent with the known orderings among dose combinations. In general, we assume that there are $k$ pre-specified dose combinations $d_{1}, d_{2}, \ldots, d_{k}$. Denote the dose for the $j t h$ entered patient as $X_{j}$, $j=1,2, \ldots, n$, which takes value $x_{j} \in\left\{d_{1}, \ldots, d_{k}\right\}$. Suppose that a continuous 
biomarker $Z$ that is informative for toxicity outcome exists. Denote the biomarker of the $j t h$ entered patient as $Z_{j} . Z_{j}$ is a random variable, but its value $z_{j}$ is known when the patient enters the trial. Denote $Y_{j}$ be an indicator for whether or not the $j t h$ entered patient experiences a DLT, which is a binary random variable, where 1 denotes the observation of a DLT and 0 denotes the observation of no DLT.

Each particular order $m, m=1, \ldots, M$ is considered as one model. For a particular ordering $m$, we model the probability of toxicity $R\left(x_{j}, z_{j}\right)$ for the $j t h$ patient with two parameters $a$ and $b$ via

$$
R\left(x_{j}, z_{j}\right)=\operatorname{Pr}\left(Y_{j}=1 \mid X_{j}=x_{j}, Z_{j}=z_{j}\right)=E\left(Y_{j} \mid x_{j}, z_{j}\right)=\psi_{m}\left(x_{j}, z_{j}, a, b\right)
$$

for some working dose-toxicity model $\psi_{m}\left(x_{j}, z_{j}, a, b\right)$ and $a \in \mathcal{A}, b \in \mathcal{B}$.

We use the empiric model as the working model

$$
\psi_{m}\left(d_{i}, z, a, b\right)=\alpha_{m i}^{\exp (a+b z)}
$$

where $\alpha_{m i}$ are the pre-specified skeleton for each treatment $d_{i}$ under model $m$ and the ordering of $\alpha_{m i}$ is consistent with the ordering of each treatment under model $m$ and $\min \left\{\alpha_{m i}\right\}>0$ and $\max \left\{\alpha_{m i}\right\}<1$. For example, if the ordering under model $m$ is $d_{1} \rightarrow d_{2} \rightarrow d_{3} \rightarrow d_{5} \rightarrow d_{4} \rightarrow d_{6}, 0<\alpha_{m 1}<\alpha_{m 2}<\alpha_{m 3}<\alpha_{m 5}<\alpha_{m 4}<\alpha_{m 6}<1$.

As Wages, Conaway and O'Quigley (2011), we assign a prior probability of each model $p(m)=\{p(1), \ldots, p(M)\}$, where $p(m) \geq 0$ and $\sum_{m=1}^{M} p(m)=1$ to take account of the prior information about the plausibility of each model. When there is no prior information available on the possible orders, we can formally specify a discrete uniform distribution for $p(m)$, where $p(m)=\frac{1}{m}, m=1, \ldots, M$.

After inclusion of the first $j$ patients into the trial, we have data in the form of 
$\Omega_{j}=\left\{x_{1}, y_{1}, z_{1}, \ldots, x_{j}, y_{j}, z_{j}\right\}$. Under model $m$, we can generate estimates $\hat{a}_{m}$ and $\hat{b}_{m}$ for the parameters $a$ and $b$. Given the estimates $\hat{a}_{m}$ and $\hat{b}_{m}$ under model $m$, we can generate estimates of the probability of toxicity by $\hat{R}\left(d_{i}, z\right)=\psi_{m}\left(d_{i}, z, \hat{a}, \hat{b}\right)=$

$\alpha_{m i}^{\exp (\hat{a}+\hat{b} z)},(i=1, \ldots, k$ and $\forall z \in \mathcal{Z})$ for each of the $k$ treatments and any value of biomarker $z$.

Denote the pre-specified target toxicity probability as $\theta$. Suppose the biomarker of the $(j+1) t h$ entered patient takes value $z_{j+1}$, we choose the order $m$ with largest posterior probability and the target dose to treat the patient has a corresponding toxicity probability as close as possible to the target toxicity rate $\theta$. The treatment $x_{j+1}$ assigned to the $(j+1) t h$ entered patient minimizes the loss function:

$$
\Delta\left(\hat{R}\left(d_{i}, z_{j+1}\right), \theta\right)=\left|\hat{R}\left(d_{i}, z_{j+1}\right)-\theta\right|, \quad i=1,2, \ldots, k
$$

which is the absolute distance between $\hat{R}\left(d_{i}, z_{j+1}\right)$ and $\theta$.

The design is a sequential updating design. After inclusion of a predetermined sample size of $n$ patients, the order $m$ with largest posterior probability is chosen among the $M$ candidate models and the treatment assigned to the hypothetical $(n+1) t h$ patient with biomarker $z_{n+1}$ is the current best estimate for the MTD.

\subsubsection{Inference}

We assign prior probability distributions $f_{0}(a)$ and $f_{1}(b)$ for the parameters $a$ and $b$ and a prior probability $p(m)$ for each model. We begin the trial by choosing the order $m$ with the largest prior probability $p(m)$ among the $M$ models. If several or all of the models have the same prior probability, we will choose at random from these models.

In order to establish estimates of the probability of toxicity for each treatment, we 
need an expression of the joint likelihood for the parameters $a$ and $b$. After inclusion of the first $j$ patients into the study, the joint likelihood for the parameters $a$ and $b$ under model $m$ is given by:

$$
L_{m}\left(a, b \mid \Omega_{j}\right)=\prod_{l=1}^{j} \psi_{m}^{y_{l}}\left(x_{l}, z_{l}, a, b\right)\left\{1-\psi_{m}\left(x_{l}, z_{l}, a, b\right)\right\}^{\left(1-y_{l}\right)}
$$

which can be used to generate estimates $\hat{a}_{m}$ and $\hat{b}_{m}$ for $a$ and $b$.

Given the data set $\Omega_{j}$ and the joint likelihood, the posterior densities for $a$ and $b$ under model $m$ are given by:

$$
\begin{aligned}
f_{m}\left(a \mid \Omega_{j}\right) & =\frac{\int_{b \in \mathcal{B}} L_{m}\left(a, b \mid \Omega_{j}\right) f_{1}(b) d b}{\iint_{a \in \mathcal{A}, b \in \mathcal{B}} L_{m}\left(a, b \mid \Omega_{j}\right) f_{0}(a) f_{1}(b) d a d b} \\
f_{m}\left(b \mid \Omega_{j}\right) & =\frac{\int_{a \in \mathcal{A}} L_{m}\left(a, b \mid \Omega_{j}\right) f_{0}(a) d a}{\iint_{a \in \mathcal{A}, b \in \mathcal{B}} L_{m}\left(a, b \mid \Omega_{j}\right) f_{0}(a) f_{1}(b) d a d b} .
\end{aligned}
$$

The posterior mean can be used as the estimates for $a$ and $b$ under model $m$ :

$$
\begin{aligned}
\hat{a}_{m}= & \frac{\iint_{a \in \mathcal{A}, b \in \mathcal{B}} a L_{m}\left(a, b \mid \Omega_{j}\right) f_{0}(a) f_{1}(b) d a d b}{\iint_{a \in \mathcal{A}, b \in \mathcal{B}} L_{m}\left(a, b \mid \Omega_{j}\right) f_{0}(a) f_{1}(b) d a d b} \\
\hat{b}_{m}= & \frac{\iint_{a \in \mathcal{A}, b \in \mathcal{B}} b L_{m}\left(a, b \mid \Omega_{j}\right) f_{0}(a) f_{1}(b) d a d b}{\iint_{a \in \mathcal{A}, b \in \mathcal{B}} L_{m}\left(a, b \mid \Omega_{j}\right) f_{0}(a) f_{1}(b) d a d b} .
\end{aligned}
$$

Furthermore, the joint likelihood can be used to establish the posterior probabilities 
of the models given the data set:

$$
\pi\left(m \mid \Omega_{j}\right)=\frac{p(m) \iint_{a \in \mathcal{A}, b \in \mathcal{B}} L_{m}\left(a, b \mid \Omega_{j}\right) f_{0}(a) f_{1}(b) d a d b}{\sum_{m=1}^{M} p(m) \iint_{a \in \mathcal{A}, b \in \mathcal{B}} L_{m}\left(a, b \mid \Omega_{j}\right) f_{0}(a) f_{1}(b) d a d b}
$$

The prior probabilities $p(m)$ for each order $m$ are sequentially updated by the toxicity response data $\Omega_{j}$. It is expected that the more data support the order $m$, the greater its posterior probability will be. When a new patient is to be enrolled, we choose a single ordering $h$ with the largest posterior probability and the corresponding working model $\psi_{h}\left(d_{i}, z, a, b\right)$ and apply a Bayesian form of the biomarker design.

Given the ordering $h$ and estimates $\hat{a}_{h}$ and $\hat{b}_{h}$, we can generate estimates of probability of toxicity for each treatment and any value of biomarker. Beginning with prior probability $f_{0}(a), f_{1}(b)$ and $p(m)$, after inclusion of the first $j$ patients into the trial, for the $(j+1)$ th enrolled patient with biomarker $z_{j+1}$, we choose the order $h$ with the largest posterior probability and compute the posterior probability of DLT for each $d_{i}$ under model $h$ :

$$
\hat{R}\left(d_{i}, z_{j+1}\right)=\psi_{h}\left(d_{i}, z_{j+1}, \hat{a}_{h}, \hat{b}_{h}\right)
$$

The treatment $x_{j+1} \in\left\{d_{1}, \ldots, d_{k}\right\}$ assigned to the $(j+1)$ th enrolled patient is the treatment $d_{i}$, such that the loss function (4.3) is a minimum. For a patient with biomarker $z$, the MTD is the recommended treatment $d_{i}$ such that the loss function (4.3) is minimized after the inclusion of the predetermined sample size of $n$ patients. 


\subsubsection{Additional Randomization for Order Selection}

Wages, Conaway and O'Quigley (2011) pointed out a dilemma of order selection with the largest posterior probability and proposed a solution of additional randomization to deal with the difficulty. Information of an order has to be obtained through experiment. Early in the trial, there may be only a negligible difference between the largest prior or posterior probability and another value in the prior or posterior probability. Without further randomization, the order with the greatest probability may be over chosen and even worse some orders may never be tried.

Additional randomization is necessary to obtain information of competing orderings. We do not rely entirely on the maximum of the posterior probability as the guidance to the most appropriate order but generate a random number $h$, $1 \leq h \leq M$, with weight of its prior probability $p(m)$ or posterior probability $\pi\left(m \mid \Omega_{j}\right)$ and implement the working model $\psi_{h}\left(d_{i}, z, a, b\right)$ associated with the order $h$. It is expected that as the trial gets underway, the accrued information will create a large gap between the values of posterior probabilities, further increasing the possibility of choosing the order with the largest posterior probability.

We do not apply the randomization algorithm for the final recommendation of the MTD. After inclusion of $n$ patients, we always choose the order $h$ with the largest posterior probability among the $M$ candidate models.

\subsection{Numerical Studies}

To illustrate the proposed method for partial order trial, consider example 1 given in Section 1.3. There are $k=6$ treatments $d_{1}, \ldots, d_{6}$ in the trial. The partial order in this trial has associated with 6 possible simple orders. Suppose the order $m=1$ is the true dose-toxicity order. In this example, the trial requires the investigation 
of the following six simple orders:

$$
\begin{aligned}
& M_{1}: d_{1} \rightarrow d_{2} \rightarrow d_{3} \rightarrow d_{4} \rightarrow d_{5} \rightarrow d_{6} \\
& M_{2}: d_{1} \rightarrow d_{2} \rightarrow d_{3} \rightarrow d_{5} \rightarrow d_{4} \rightarrow d_{6} \\
& M_{3}: d_{1} \rightarrow d_{2} \rightarrow d_{3} \rightarrow d_{5} \rightarrow d_{6} \rightarrow d_{4} \\
& M_{4}: d_{1} \rightarrow d_{2} \rightarrow d_{5} \rightarrow d_{6} \rightarrow d_{3} \rightarrow d_{4} \\
& M_{5}: d_{1} \rightarrow d_{2} \rightarrow d_{5} \rightarrow d_{3} \rightarrow d_{6} \rightarrow d_{4} \\
& M_{6}: d_{1} \rightarrow d_{2} \rightarrow d_{5} \rightarrow d_{3} \rightarrow d_{4} \rightarrow d_{6}
\end{aligned}
$$

Other settings including the true probability of toxicity, the target toxicity probability, the choice of skeleton for the working model, the prior for $a$ and $b$ and the distributions for the biomarker remain the same as those specified in Section 2.2.

We consider two examples of the true probability of toxicity.

In the first example, suppose the true probability of toxicity follows an empiric model:

$$
R\left(d_{i}, z\right)=\beta_{i}^{\exp (a+b z)}
$$

where $\beta_{1}=0.04, \beta_{2}=0.07, \beta_{3}=0.20, \beta_{4}=0.35, \beta_{5}=0.55$ and $\beta_{6}=0.70$. In this example, the form of the true probability of toxicity is similar to that of the working model.

In the second example, with the aim of further validating the robustness of the proposed design, we investigate the true probability of toxicity following a logit model:

$$
R\left(d_{i}, z\right)=\frac{\exp \left(a_{i}-b z\right)}{1+\exp \left(a_{i}-b z\right)}
$$

where $i=1, \ldots, 6$ and we study two scenarios of $a_{i}$, shown in Table 4.1. 
Table 4.1: Scenarios of $a_{i}$

\begin{tabular}{ccccccc}
\hline & $a_{1}$ & $a_{2}$ & $a_{3}$ & $a_{4}$ & $a_{5}$ & $a_{6}$ \\
\hline Scenario 1 & -1.8 & -1.4 & -0.5 & 0.1 & 0.8 & 1.4 \\
Scenario 2 & -4.9 & -4 & -2.4 & -1.4 & -0.4 & 0.3 \\
\hline
\end{tabular}

The values of $a_{i}$ are taken such that for scenario $1, \beta_{i}^{\exp (-0.5)}=\frac{\exp \left(a_{i}\right)}{1+\exp \left(a_{i}\right)}, i=$ $1, \ldots, 6$ and for scenario $2, \beta_{i}^{\exp (0.42)}=\frac{\exp \left(a_{i}\right)}{1+\exp \left(a_{i}\right)}, i=1, \ldots, 6$, i.e. for biomarker with value 0 , the true probability of toxicity in the two examples are the same. In the second example, the form of the true probability of toxicity is different from that of the working model.

The logit model is less steep than the empiric model when the coefficients of the biomarker are the same in the two models. The logit model is less sensitive to the change of the biomarker while the empiric model is more sensitive to the change of the biomarker.

We investigate a variety of toxicity scenarios. For the true probability of toxicity with an empiric model, we study several different true values of $a$ and $b$, where $a=-0.5$ and 0.42 and $b=1.5,1,0.5,0.3,0.1,-0.1,-0.3,-0.5,-1$ and -1.5 . For the true probability of toxicity with a logit model, we study the two scenarios of $a_{i}$ in Table 4.1 and $b=1.5,1,0.5,0.3,0.1,-0.1,-0.3,-0.5,-1$ and -1.5 .

Suppose the target toxicity probability is $\theta=0.20$.

We use the skeleton $\{0.020,0.081,0.200,0.356,0.515,0.654\}$ and accommodate the skeleton for each possible ordering in the working model based on the ordering information of each possible order. The skeleton for each possible order is shown in Table 4.2 .

We assign a standard normal prior $N(0,1)$ for a similar to O'Quigley and Shen (1996). Generally, before we begin the trial, we may have quite strong knowledge concerning whether an increased biomarker value indicates a higher probability of 
Table 4.2: Skeleton for the six possible orders

\begin{tabular}{lcccccc}
\hline & $d_{1}$ & $d_{2}$ & $d_{3}$ & $d_{4}$ & $d_{5}$ & $d_{6}$ \\
\hline$m=1$ & 0.020 & 0.081 & 0.200 & 0.356 & 0.515 & 0.654 \\
$m=2$ & 0.020 & 0.081 & 0.200 & 0.515 & 0.356 & 0.654 \\
$m=3$ & 0.020 & 0.081 & 0.200 & 0.654 & 0.356 & 0.515 \\
$m=4$ & 0.020 & 0.081 & 0.515 & 0.654 & 0.200 & 0.356 \\
$m=5$ & 0.020 & 0.081 & 0.356 & 0.654 & 0.200 & 0.515 \\
$m=6$ & 0.020 & 0.081 & 0.356 & 0.515 & 0.200 & 0.654 \\
\hline
\end{tabular}

toxicity or a lower probability of toxicity. We assign a truncated normal prior for $b$. When we believe the probability of toxicity is decreasing with an increased biomarker value, corresponding to a true positive value of $b$ in the simulation, we assign a truncated normal prior for $b$ with the domain on $\mathbf{R}^{+}$:

$$
f_{1}(b)=\left\{\begin{array}{cc}
\frac{2}{\sqrt{2 \pi}} \exp \left(-\frac{b^{2}}{2}\right) & b \geq 0 \\
0 & b<0
\end{array}\right.
$$

When we believe the probability of toxicity is increasing with an increased biomarker value, corresponding to a true negative value of $b$ in the simulation, we assign a truncated normal prior for $b$ with the domain on $\mathbf{R}^{-}$:

$$
f_{1}(b)=\left\{\begin{array}{cc}
0 & b>0 \\
\frac{2}{\sqrt{2 \pi}} \exp \left(-\frac{b^{2}}{2}\right) & b \leq 0
\end{array}\right.
$$

We use a predetermined sample size of patients $n=25$ in the simulation. We define a measurement to evaluate the performance of the proposed design. After inclusion of the predetermined sample size of $n$ patient, for the hypothetical $(n+1)$ th patient with biomarker $z_{n+1}$, correct recommendation refers to the MTD recommended by the proposed design being the same as the true MTD corresponding to the value of biomarker. We further define the overall probability of correct 
recommendation as:

$$
\begin{aligned}
& P(\text { correct MTD recommendation }) \\
= & \int P(\text { correct MTD recommendation } \mid Z=z) f_{Z}(z) d z,
\end{aligned}
$$

where $f_{Z}(z)$ is the probability density function of biomarker $Z$.

To evaluate the operating characteristics and performance of the proposed method, we investigate biomarkers with different distributions. We first investigate a biomarker with a standard normal distribution $Z \sim N(0,1)$. According to genetic study, a toxicity biomarker may have a mixture distribution of a point mass at 0 and a distribution defined on $\mathbf{R}^{+}$. We then investigate biomarkers with such distributions. For the point mass at 0 , we investigate two weights 0.3 and 0.6. For distributions defined on $\mathbf{R}^{+}$, we choose truncated normal distribution and log-normal distribution with $\mu=0$ and $\sigma=1$. The four specified mixture distributions are as follows:

$$
\begin{aligned}
& \mathrm{w}=0.3 \text { truncated normal } \\
& f_{Z}(z)=0.7 \times \frac{2}{\sqrt{2 \pi}} \exp \left(-\frac{1}{2} z^{2}\right) \text { for } z>0 \\
& \mathrm{w}=0.6 \text { truncated normal } \\
& \operatorname{Pr}(Z=0)=0.6 \\
& f_{Z}(z)=0.4 \times \frac{2}{\sqrt{2 \pi}} \exp \left(-\frac{1}{2} z^{2}\right) \text { for } z>0 \\
& \mathrm{w}=0.3 \log \text {-normal } \\
& \operatorname{Pr}(Z=0)=0.3 \\
& f_{Z}(z)=0.7 \times \frac{1}{z \sqrt{2 \pi}} \mathrm{e}^{-\frac{(\ln z)^{2}}{2}} \text { for } z>0 \\
& \mathrm{w}=0.6 \log \text {-normal } \\
& \operatorname{Pr}(Z=0)=0.6 \\
& f_{Z}(z)=0.4 \times \frac{1}{z \sqrt{2 \pi}} \mathrm{e}^{-\frac{(\ln z)^{2}}{2}} \text { for } z>0
\end{aligned}
$$


Under all scenarios, 1000 trials were simulated. In this thesis, the "biomarker design for single-agent trial" proposed in Section 2.1 is synonymous with "biomarker design for simple order". The "standard CRM for single-agent trial" is synonymous with "non-biomarker design for simple order". The "biomarker design for multipleagent trial" proposed in Section 4.1 is synonymous with "biomarker design for partial order". The "partial order CRM" proposed by Wages, Conaway and O'Quigley's method (2011) introduced in Section 1.4.4 is synonymous with "non-biomarker design for partial order".

On one hand, we compare the proposed biomarker design for partial order with the partial order CRM. The partial order CRM refers to Wages, Conaway and O'Quigley's method (2011) whose working model does not contain biomarker although the true toxicity response is assumed to be heterogeneous in the simulation. This comparison shows how necessary the biomarker information should be included.

On the other hand, we compare the proposed biomarker design for partial order with the biomarker design for simple order. When the correct toxicity ordering is known, the method simplifies to the biomarker design for simple order in Section 2.1. The probability of correct MTD recommendation for the biomarker design for simple order, corresponding to where the true dose-toxicity order is fully known, provides a benchmark of how well the biomarker design for partial order performs. It is unreasonable to expect a design for a partially known order to perform as well as one for a fully known order. The comparison between the biomarker design for partial order and the biomarker design for fully known order is useful in assessing how much information is lost as a result of not having fully known order.

In the example of the true probability of toxicity with the empiric model (4.9), Figure A.1 to A.4 provide the probability of correct MTD recommendation. Figure A.5 to A.8 provide the corresponding percentage of patients treated at MTDs. 
For the comparison between the biomarker design for partial order and the partial order CRM, in terms of the probability of correct MTD recommendation, when the biomarker is strong, corresponding to a large true absolute value of $b$ in the simulations, the proposed biomarker design for partial order yields a higher probability than the partial order CRM. When the biomarker is weak, for example the scenarios with

(1) $a=-0.5, b=0.3$ and biomarker with truncated normal $w=0.6$

(2) $a=-0.5, b=0.1$ and biomarker with truncated normal $w=0.3$

(3) $a=-0.5, b=0.1$ and biomarker with truncated normal $w=0.6$

(4) $a=-0.5, b=0.1$ and biomarker with log-normal $w=0.3$

(5) $a=-0.5, b=0.1$ and biomarker with log-normal $w=0.6$

(6) $a=0.42, b=0.5$ and biomarker with truncated normal $w=0.3$

(7) $a=0.42, b=0.5$ and biomarker with truncated normal $w=0.6$

(8) $a=0.42, b=0.3$ and biomarker with truncated normal $w=0.3$

(9) $a=0.42, b=0.3$ and biomarker with truncated normal $w=0.6$

(10) $a=0.42, b=0.3$ and biomarker with log-normal $w=0.6$

(11) $a=0.42, b=0.1$ and biomarker with standard normal distribution

(12) $a=0.42, b=0.1$ and biomarker with truncated normal $w=0.3$

(13) $a=0.42, b=0.1$ and biomarker with truncated normal $w=0.6$

(14) $a=0.42, b=0.1$ and biomarker with log-normal $w=0.3$ 
(15) $a=0.42, b=0.1$ and biomarker with log-normal $w=0.6$

(16) $a=0.42, b=-0.1$ and biomarker with standard normal distribution

(17) $a=0.42, b=-0.1$ and biomarker with truncated normal $w=0.3$

(18) $a=0.42, b=-0.1$ and biomarker with truncated normal $w=0.6$

partial order CRM yields slightly higher probability than the biomarker design for partial order. Yet, the probability of correct MTD recommendation yielded by the biomarker design for partial order is also very high.

In terms of the percentage of patients treated at the MTDs, when the biomarker is strong, the biomarker design for partial order treats more patients at the MTDs than the partial order CRM. When the biomarker is weak, for example the scenarios with

(1) $a=-0.5, b=0.3$ and biomarker with truncated normal $w=0.3$

(2) $a=-0.5, b=0.3$ and biomarker with truncated normal $w=0.6$

(3) $a=-0.5, b=0.3$ and biomarker with log-normal $w=0.6$

(4) $a=-0.5, b=0.1$ and biomarker with truncated normal $w=0.3$

(5) $a=-0.5, b=0.1$ and biomarker with truncated normal $w=0.6$

(6) $a=-0.5, b=0.1$ and biomarker with log-normal $w=0.3$

(7) $a=-0.5, b=0.1$ and biomarker with log-normal $w=0.6$

(8) $a=0.42, b=0.5$ and biomarker with truncated normal $w=0.3$

(9) $a=0.42, b=0.5$ and biomarker with truncated normal $w=0.6$ 
(10) $a=0.42, b=0.3$ and biomarker with standard normal

(11) $a=0.42, b=0.3$ and biomarker with truncated normal $w=0.3$

(12) $a=0.42, b=0.3$ and biomarker with truncated normal $w=0.6$

(13) $a=0.42, b=0.3$ and biomarker with log-normal $w=0.3$

(14) $a=0.42, b=0.3$ and biomarker with log-normal $w=0.6$

(15) $a=0.42, b=0.1$ and biomarker with standard normal distribution

(16) $a=0.42, b=0.1$ and biomarker with truncated normal $w=0.3$

(17) $a=0.42, b=0.1$ and biomarker with truncated normal $w=0.6$

(18) $a=0.42, b=0.1$ and biomarker with log-normal $w=0.3$

(19) $a=0.42, b=0.1$ and biomarker with log-normal $w=0.6$

(20) $a=0.42, b=-0.1$ and biomarker with standard normal distribution

(21) $a=0.42, b=-0.1$ and biomarker with truncated normal $w=0.3$

(22) $a=0.42, b=-0.1$ and biomarker with truncated normal $w=0.6$

(23) $a=0.42, b=-0.1$ and biomarker with log-normal $w=0.3$

(24) $a=0.42, b=-0.1$ and biomarker with log-normal $w=0.6$

partial order CRM treats slightly more patients at the MTDs than the biomarker design for partial order.

For the comparison between the biomarker design for partial order and the biomarker design for simple order, in the simulation results, the biomarker design 
for partial order usually yields a slightly smaller probability of correct MTD recommendation than the biomarker design for simple order. This accords with our intuition that a design for a partially known order perform less well than a design for a fully known order. In terms of the percentage of patients treated at the MTDs, the biomarker design for partial order usually treats less patients at the MTDs than the biomarker design for simple order.

The simulation result indicates that the proposed biomarker design for partial order is not quite sensitive to the distribution of biomarker. In the example of the true probability of toxicity with the logit model (4.10), Figure A.5 to A.8 provide the probability of correct MTD recommendation. Figure A.13 to A.16 provide the corresponding percentage of patients treated at MTDs. Similarly, when the biomarker is strong, the biomarker design for partial order performs better than the partial order CRM. When the biomarker is weak, the partial order CRM may perform slightly better than the biomarker design for partial order. In addition, the biomarker design for partial order usually performs a little worse than the biomarker design for simple order. The simulation result indicates that the biomarker design for partial order is not quite sensitive to the underlying true probability of toxicity and demonstrates the robustness of the method.

Overall, the simulation results indicate that, in terms of identifying MTDs, the proposed biomarker design for partial order demonstrates superior performance when the biomarker is strong and the performance of the proposed biomarker design is comparable to that of partial order CRM when the biomarker is weak. The simulation results support the feasibility and necessity of choosing a distinct MTD for each individual patient by use of toxicity biomarker. 


\subsection{Model Selection between Biomarker Model and Non-biomarker Model}

\subsubsection{Statistical Method}

The simulation result in Section (4.2) indicates that when the biomarker is strong the biomarker design for partial order proposed in Section (4.1) performs better, when the biomarker is weak the partial order CRM introduced in Section (1.4.4), i.e. the non-biomarker model for partial order, performs better.

When the toxicity response is homogeneous, O'Quigley, Pepe and Fisher (1990) pointed out a one-parameter working model such as $\psi\left(d_{i}, a\right)=\alpha_{i}^{\exp (a)}$ is a underparameterized model. They compared the performance of a one-parameter model with a two-parameter model. Generally, the one-parameter model performs better than a two-parameter model.

When the toxicity response is heterogenous, the biomarker model for partial order is more flexibility than the non-biomarker model for partial order in identifying the MTDs. The non-biomarker model for partial order is relatively underparameterized. Under each possible simple order, it chooses a unique MTD for all values of biomarker. The non-biomarker model leads to more handicap in trying to find a good fit and a correct MTD for some values of biomarker, even if the correct simple order is chosen. However, when the biomarker is weak, most values of biomarker may have the same MTD. Wages, Conaway and O'Quigley (2011) pointed out the identification of the correct simple order is not the main concern for partial order CRM. Even if the correct simple order is not selected, partial order CRM still efficiently identify the MTD. In this situation, when the biomarker is weak, the lack of flexibility of the non-biomarker model for partial order may turn into 
an advantage in identifying a correct MTD for most value of biomarker, especially considering the small sample size of patients and vague prior of the parameters. For each possible simple order, at least for the correct simple order, the non-biomarker model for partial order may be considered as the better model than the biomarker model for partial order when the biomarker is weak. When the biomarker is strong, the correct MTDs spread out for different values of biomarker, so for each possible simple order the biomarker model is considered better while the non-biomarker model is lack of flexibility.

This section has the appeal of implementing model selection techniques. If the model selection technique can effectively choose the better model between the biomarker design for partial order and the non-biomarker design for simple order, our approach will identify MTDs more effectively. Schwarz (1978) derived the Bayesian information criterion (BIC) given by:

$$
B I C=-2 \log \left(L\left(\mathbf{a} \mid \Omega_{j}\right)\right)+K \log (n),
$$

where $\mathbf{a}$ is the estimable parameters and $K$ is the number of estimable parameters. In the biomarker design for partial order, under a particular order $h$, two parameters $a_{b i o, h}$ and $b_{b i o, h}$ are estimated, $K=2$ and the BIC for the order $h$ is given by:

$$
B I C_{b i o, h}=-2 \log \left(L_{h}\left(\hat{a}_{b i o, h}, \hat{b}_{b i o, h} \mid \Omega_{j}\right)\right)+2 \log (n)
$$

For the order $h$, the expression $L_{h}\left(\hat{a}_{b i o, h}, \hat{b}_{b i o, h} \mid \Omega_{j}\right)$ is the value of the likelihood evaluated at the point of posterior mean $\hat{a}_{b i o, h}$ and $\hat{b}_{b i o, h}$. In the non-biomarker design, the working model is $\psi_{h}\left(d_{i}, a\right)=\alpha_{h i}^{\exp (a)}$, under a particular order $h$, one 
parameter $a_{n o n, h}$ is estimated, $K=1$ and the BIC for the order $h$ is given by:

$$
B I C_{n o n, h}=-2 \log \left(L_{h}\left(\hat{a}_{n o n, h} \mid \Omega_{j}\right)\right)+\log (n) .
$$

For the order $h$, the expression $L_{h}\left(\hat{a}_{n o n, h} \mid \Omega_{j}\right)$ is the value of the likelihood evaluated at the posterior mean $\hat{a}_{n o n, h}$.

For the biomarker working model, we choose an order $h_{b i o}$ according to the corresponding posterior probability of each possible simple order. Similarly, for the non-biomarker model, we choose an order $h_{n o n}$ according to the corresponding posterior probability of each possible simple order. The posterior probability of each possible simple order for the biomarker working model and that for the nonbiomarker working model could be different. Even if they are the same, $h_{b i o}$ and $h_{\text {non }}$ could be different. As Wages, Conaway and O'Quigley (2011) pointed out the choice of an order is not the main concern, we compare the biomarker model under the order $h_{b i o}$ and the non-biomarker model under the order $h_{n o n}$. The model with smaller BIC is considered better and will be selected.

Before the first patient is enrolled, we do not have the estimates $\hat{a}_{b i o, h}, \hat{b}_{b i o, h}$ and $\hat{a}_{n o n, h}$. We simply choose the non-biomarker model as the working model and choose an order $h_{n o n}$ according to the prior probability of each possible simple order by the randomization method for order selection proposed in Section (4.1.3). When the estimates $\hat{a}_{b i o, h}, \hat{b}_{b i o, h}$ and $\hat{a}_{n o n, h}$ are all achieved, we are able to compute and compare the BIC for the biomarker model and the non-biomarker model. In the Bayesian framework, we obtain the posterior mean $\hat{a}_{b i o, h}, \hat{b}_{b i o, h}$ and $\hat{a}_{n o n, h}$ as soon as the response of whether or not the first patient experiences a DLT is observed. After that, the BIC is computed for the biomarker model and the non-biomarker model and under selected orders the model with the smaller value of BIC could be 
selected.

After inclusion of the first $j$ patients into the trial, for each possible simple order, we estimate both the biomarker model and the non-biomarker model and generate estimates $\hat{a}_{b i o, h}, \hat{b}_{b i o, h}$ and $\hat{a}_{n o n, h}$. When the $(j+1) t h$ patient is enrolled, for the biomarker working model, we choose an order $h_{b i o}$ according to the corresponding posterior probability of each possible simple order by the randomization method for order selection proposed in Section (4.1.3). Similarly, for the non-biomarker model, we choose an order $h_{\text {non }}$ according to the corresponding posterior probability of each possible simple order by the randomization method for order selection. The model with smaller BIC between the biomarker model under the order $h_{b i o}$, i.e. $\psi_{h_{b i o}}\left(d_{i}, z, a, b\right)=\alpha_{h_{b i o} i}^{\exp (a+b z)}$ and the non-biomarker model under the order $h_{n o n}$, i.e. $\psi_{h_{\text {non }}}\left(d_{i}, a\right)=\alpha_{h_{\text {non } i}}^{\exp (a)}$ is considered better and will be selected. Then select the treatment $x_{j+1}$ assigned to the $(j+1)$ th entered patient which minimizes the loss function (4.3).

After inclusion of a predetermined sample size of $n$ patients, we no longer use the randomization algorithm to select an order. We choose the model with smaller BIC between the biomarker model with the largest corresponding posterior probability and the non-biomarker model with the largest corresponding posterior probability and choose the MTD for each individual patient under the working model.

\subsubsection{Numerical Studies}

In aim of improving the performance of biomarker design for partial order when the biomarker is weak, we propose the model selection method. In this section, we compare the performance of the BIC model selection method for partial order with the biomarker model for partial order and the non-biomarker model for partial. In 
this dissertation, the "BIC model selection method" proposed in Section (4.3) is synonymous with "BIC method for partial order". We do the simulation studies under the same setting as Section (4.2).

In the example of the true probability of toxicity with the empiric model (4.9), Figure A.1 to A.4 provide the probability of correct MTD recommendation. Figure A.5 to A.8 provide the corresponding percentage of patients treated at MTDs. Table A.1 provides the proportion of selecting biomarker model as the final working model after inclusion of the predetermined sample size of patients. In the example of the true probability of toxicity with the logit model (4.10), Figure A.5 to A.8 provide the probability of correct MTD recommendation. Figure A.13 to A.16 provide the corresponding percentage of patients treated at MTDs. Table A.2 provides the proportion of selecting biomarker model as the final working model

In terms of the probability of correct MTD recommendation, the probability of the BIC method for partial order is usually between the probability of the biomarker model for partial order and the non-biomarker model for partial order. When the biomarker is strong, the probability of biomarker model for partial order is larger than the non-biomarker model for partial order, the probability of the BIC method for partial order is usually slightly smaller than but approaches to that of biomarker model for partial order. It is reasonable that when the probability of biomarker model for partial order is larger than that of the non-biomarker model for partial order, the probability of the BIC method for partial order is smaller than that of biomarker model for partial order, because with the model selection mechanism the BIC method for partial order sometimes chooses the non-biomarker model for partial order, further lowering the probability of correct MTD recommendation.

When the biomarker is weak, the probability of biomarker model for partial order is sometimes smaller than that of the non-biomarker model for partial order. 
Especially, for those scenarios whose probability of biomarker model for partial order is smaller than that of non-biomarker model for partial order, the probability of BIC method for partial order is obviously larger than that of biomarker model for partial order and approaches to the probability of non-biomarker model for partial order. We conclude that the BIC method for partial order generally improves the performance in identifying MTDs when the biomarker is weak.

Similarly for the percentage of patients treated at the MTDs, when the biomarker is strong, the percentage of BIC method for partial order is usually slightly smaller than but approaches to that of biomarker model for partial order. When the biomarker is weak, the BIC method for partial order usually improves the percentage.

In terms of the proportion of selecting biomarker model as the final working model, when the biomarker is strong, the proportion is large, when the biomarker is weak, the proportion could be very small. In addition, the proportion for BIC method for partial order is usually smaller than that for the BIC method simple order.

Overall, the BIC method for partial order is a trade off between biomarker model for partial order and non-biomarker model for partial order. When the biomarker is strong, the BIC method for partial order may lower the performance a little bit. When the biomarker is weak, the BIC method for partial order improves the performance a lot. The BIC method for partial order generates a good result much more stably than the biomarker model for partial order and non-biomarker modelfor partial order whenever the biomarker is strong or weak. The simulation results indicates that it is worthwhile to replace the biomarker model for partial order with the BIC model selection method for partial order, especially when it is uncertain whether the biomarker is strong or not before the trial. Yet when it is quite certain 
that the biomarker is strong before the trial, the biomarker model for partial order is still recommended. 


\section{Chapter 5}

\section{Conclusion and Future Research}

\subsection{Conclusion}

The primary goal of a Phase I clinical trial is to estimate the maximum tolerated dose (MTD). In this dissertation, we address heterogeneous toxicity with a continuous biomarker that is informative for toxicity response, leading to distinct MTDs for each individual patient. Generally, before we begin the trial, we may have some knowledge about whether an increased biomarker value indicates a higher probability of toxicity or a lower probability of toxicity. Using the biomarker information, we choose a distinct MTD for individual patient. The goal is accurate estimation of the MTDs for individual patient, subject to the ethical constraints of treating as many patients as possible at and around the MTD. In addition, we address the consideration of a partial ordering for the probability of toxicity for available treatments in this dissertation. In single-agent trials, the order of the probability of toxicity for all the treatments is fully known, which is a "simple order". In multipleagent trials, we may be able to identify the order of the probability of toxicity for only a subset of the available treatments, which is a "partial order". We propose 
a new biomarker design for simple order and a biomarker design for partial order. The two designs which lean upon the biomarker information show themselves to be effective in estimating the MTDs in Phase I clinical trials when the toxicity biomarker is strong enough.

We have tested our methods in extensive simulation studies. The simulation results indicate that, for both simple order and partial order, when the biomarker is strong the performance of the biomarker-based methods are superior than those existing non-biomarker dose-finding methods. The showing of our methods in extensive simulation studies gives us confidence in recommending using biomarker information when the biomarker is strong enough. When the biomarker is weak, the performance of the biomarker-based methods sometimes is better while sometimes it is a little bit worse than those of the non-biomarker dose-finding methods but it is still acceptable good. Under certain conditions, we prove convergence for the biomarker design for simple order. Asymptotic normalities for the estimates of the parameters and some other properties are studied. These theoretical properties provide confidence in using the biomarker information. Therefore, we recommend using the biomarker.

The theoretical properties are established for large sample size. Yet, a Phase I trial has the characteristic of small sample size. Since the convergence could be slow, sometimes the biomarker model does not perform as well as non-biomarker model. In aim of improving the performance for a weak biomarker, we proposes a BIC model selection method to select a model between the biomarker model and non-biomarker model. Generally, the BIC method is a trade off between biomarker model and non-biomarker model. When the biomarker is strong, the BIC method may lower the performance a little bit. When the biomarker is weak, the BIC method improves the performance a lot. The BIC method generates a good result 
much more stably than the biomarker model and non-biomarker model whenever the biomarker is strong or weak. The simulation results indicates that it is worthwhile to replace the biomarker model with the BIC model selection method, especially when it is uncertain whether the biomarker is strong or not before the trial. Yet when it is quite certain that the biomarker is strong before the trial, the biomarker design is recommended.

\subsection{Further Research}

There are relatively few statistical methods for dose-finding designs that consider heterogeneous toxicity response. Until now, no other designs have been established to address more than two groups of patients. This dissertation is a starting point for the problem of heterogeneous toxicity response. There are a lot of areas for future research.

First, it is meaningful to establish the early stopping rule for biomarker design. In principle, a trial should be stopped once a sufficient amount of information about the MTD has been accumulated. In practice, investigators are likely to stop the trial if there are already many patients on a single treatment, or if a single treatment is used for many consecutive patients and this treatment is very likely to be the recommended MTD. Conaway, Dunbar and Peddada (2004) presented practical stopping rules, such as "(9, 18)" rule which stipulates that the trial be stopped if nine consecutive subjects are entered on a single treatment or if the total number of patients on one treatment is greater than 18 . Other similar rules including the "(6, $9)$ ", "(6, 12)" and " $(6,18)$ " rules are also investigated. Yet, in the design involving biomarker, patients on the same treatment may have different values of biomarker. The accumulation of experiments on a single treatment may not be sufficient to be 
a correct stopping rule.

Second, it is of great importance to establish accurate estimation for the coefficient of the biomarker, i.e. b. In the Bayesian framework, due to the prior, the estimation of the absolute value of $b$ is likely to inflate when the biomarker is weak. We may think in the way that the estimate $\hat{b}$ in the biomarker model is more far away from the truth than the estimate, i.e. 0 in the non-biomarker model. This could be an explanation why the non-biomarker model may perform better when the biomarker is weak. If we can get rid of the prior, we may obtain a more accurate estimation more quickly. A possible solution is to establish the maximum likelihood estimator. Another idea is to consider several models with pre-specified discrete value of $b$, assign equal weight for each model, and sequentially choose the model with largest likelihood. If we do have strong prior knowledge about the possible range of $b$, this idea will be recommended and intuitively it will be better than the ordinary MLE.

Third, the randomization for order selection in the design for partial ordering is always critical. We may refer to the randomization methods for partial order CRM and investigate the operating characteristic for biomarker design for partial order. The selection of a working model is much more complicated in the BIC method for partial order. We have biomarker models for each possible order and non-biomarker models for each possible order. The posterior probabilities for biomarker models are different from those for non-biomarker models. One approach is to select two orders for biomarker models and non-biomarker models respective and compare the selected biomarker model and the selected non-biomarker models. Another approach is to select a same order for biomarker models and non-biomarkers and compare the selected models. Both of the two approach has its reasoning as well as disadvantages.

Forth, a future direction for biomarker-based design is the consideration of mul- 
tiple biomarkers. It is expected that it is very difficult to achieve an accurate estimation with a very small sample size but relatively large number of covariates. Similarly to the problem of weak biomarker in the single biomarker design, we need to consider the problem of selecting strong biomarkers and excluding weak biomarkers.

Fifth, a very practical consideration of a biomarker-based design is to address categorical biomarkers, especially ordered groups of patients. If we have $k$ ordered categories, one possible solution is to build a working model with $k$ parameters. The model with $k$ parameters is flexible enough such that each category can target its correct MTD in the long run under very weak conditions. Yet, the increment of the number of parameters in the working model may lead to difficulty in estimation. In the Bayesian framework, the establishment of the prior for the parameters is the crucial question. 


\section{Appendix A}

\section{Appendix}

This section contains the results of simulation studies described in Chapter 3 . We consider two examples of the true probability of toxicity. In the first example, the true probability of toxicity follows an empiric model: $R\left(d_{i}, z\right)=\beta_{i}^{\exp (a+b z)}$. In the second example, the true probability of toxicity follows a logit model: $R\left(d_{i}, z\right)=$ $\frac{\exp \left(a_{i}-b z\right)}{1+\exp \left(a_{i}-b z\right)}$.

Figure A.1 to A.4 provide the probability of Correct MTD Recommendation for the empiric truth. Figure A.5 to A.8 provide the percentage of patients treated at the MTDs for the empiric truth. Figure A.7 to A.12 provide the probability of Correct MTD Recommendation for the logit truth. Figure A.13 to A.16 provide the percentage of patients treated at the MTDs for the logit truth.

Table A.1 provides the proportion of selecting biomarker model as final working model the empiric truth. Table A.2 provides the proportion of selecting biomarker model as final working model the logit truth. 


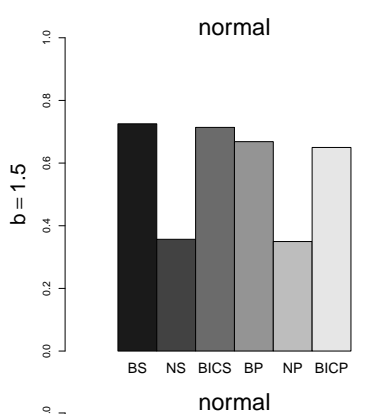

truncated normal $\mathrm{w}=0.3$ truncated normal $\mathrm{w}=0.6$

log-normal $\mathrm{w}=0.3$

log-normal $\mathbf{w}=0.6$
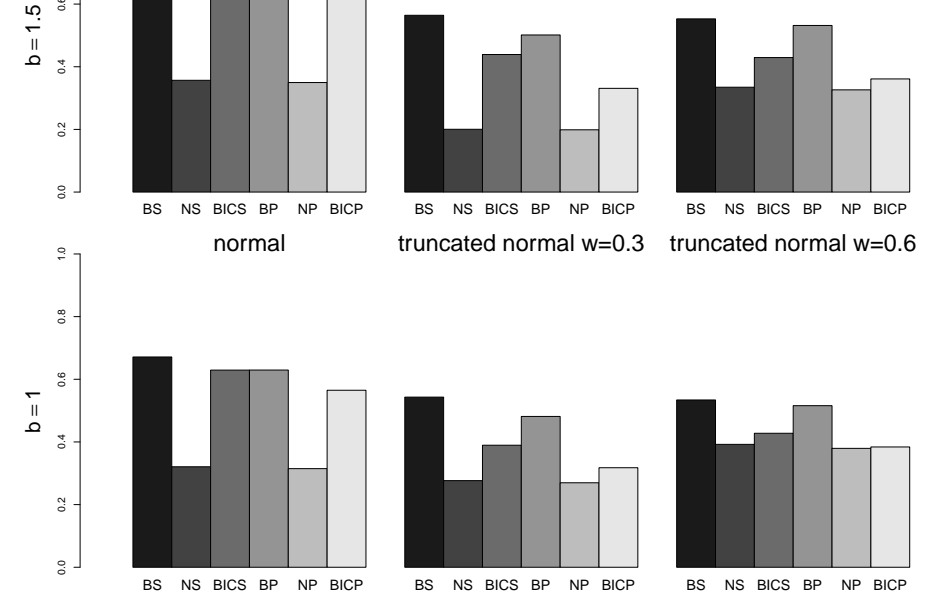

truncated normal $\mathrm{w}=0.3$ truncated normal $\mathrm{w}=0.6$
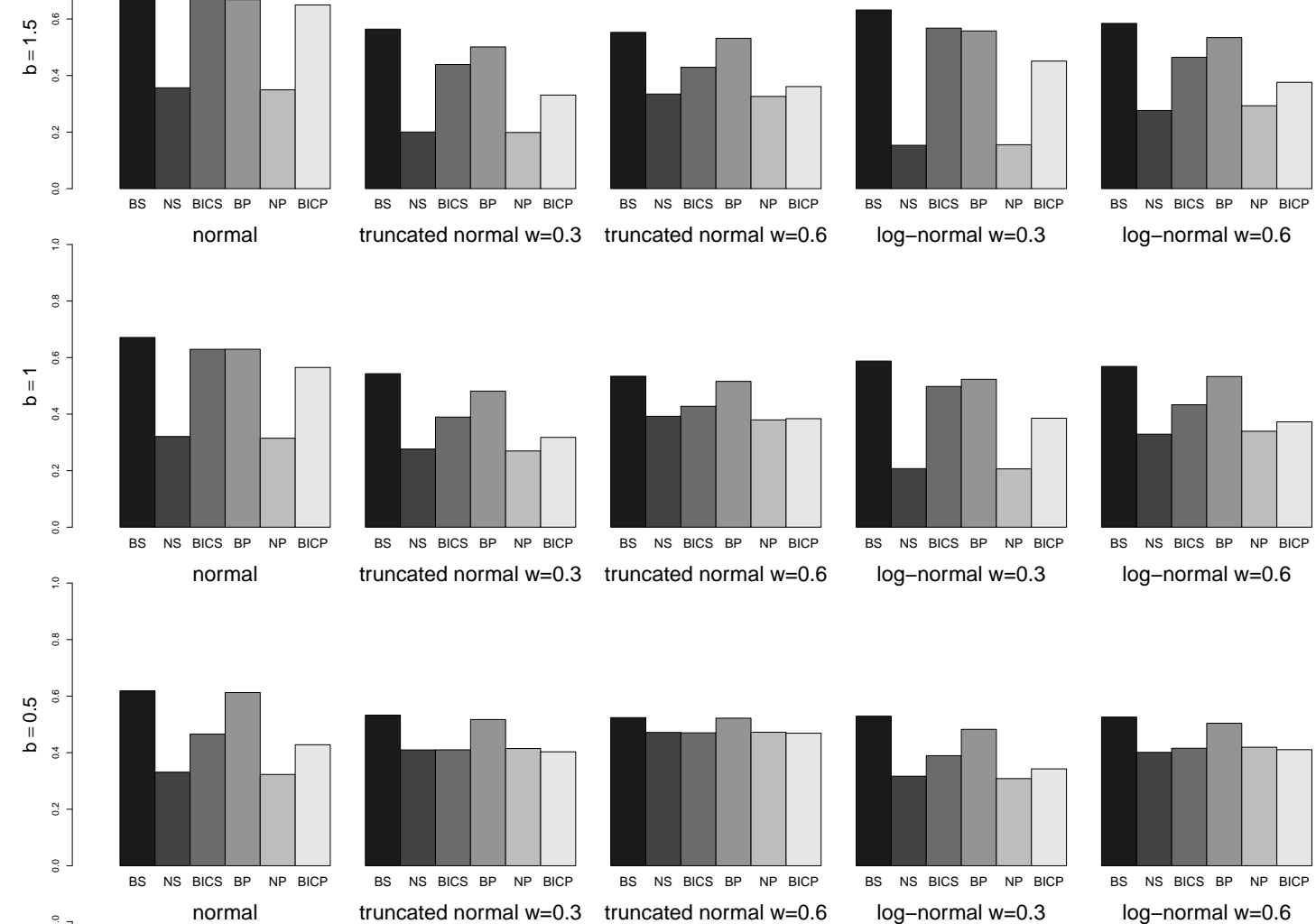

truncated normal $\mathrm{w}=0.3$ truncated normal $\mathrm{w}=0.6$
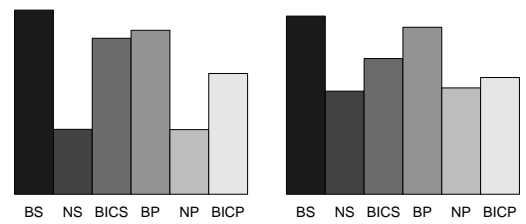

log-normal $\mathrm{W}=0.3$

log-normal $\mathrm{w}=0.6$
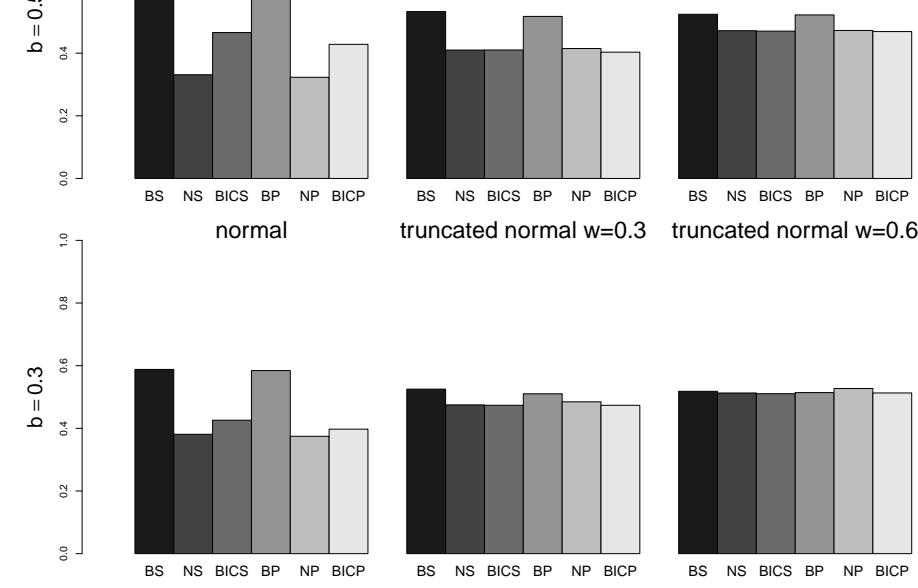

truncated normal $\mathrm{W}=0.3$

truncated normal $\mathrm{W}=0.6$
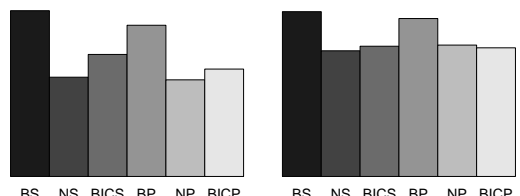

log-normal $\mathrm{w}=0.3$

log-normal $\mathbf{w}=0.6$
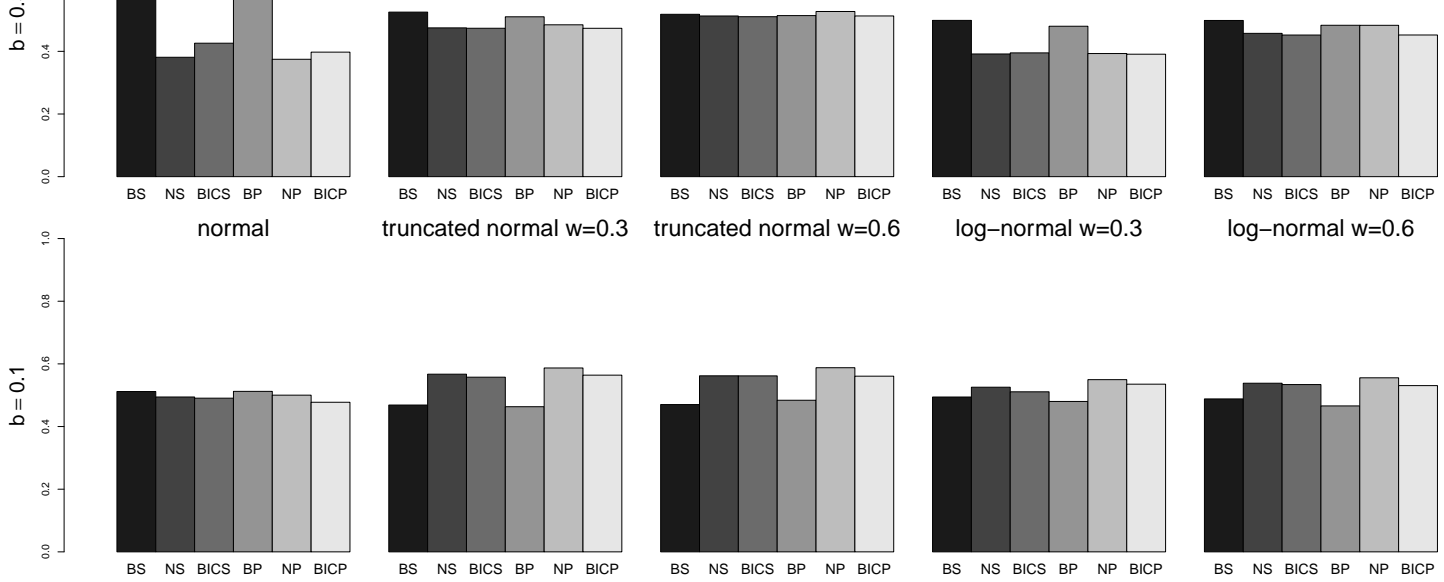

truncated normal $\mathrm{W}=0.3$ truncated normal $\mathrm{W}=0.6$

log-normal $\mathrm{w}=0.3$

log-normal $\mathrm{w}=0.6$
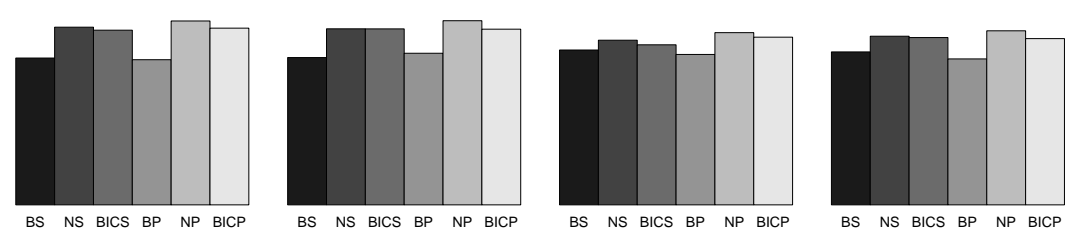

Figure A.1: The probability of Correct MTD Recommendation. The true probablity of toxicity follows an empiric model. $a=-0.5$. The labels BS NS BICS BP NP and BICP for each bar refer to using biomarker model for simple order, non-biomarker model for simple order , BIC model for simple order, biomarker model for partial order, non-biomarker model for partial order and BIC model for partial order. 


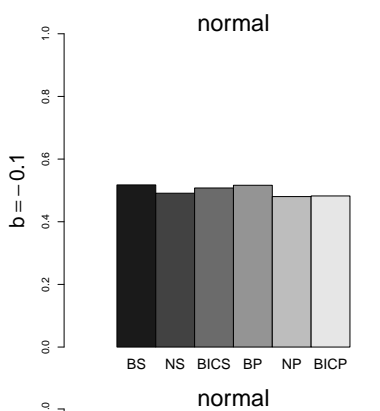

truncated normal $\mathrm{w}=0.3$ truncated normal $\mathrm{w}=0.6$

log-normal $w=0.3$

log-normal $\mathbf{w}=0.6$
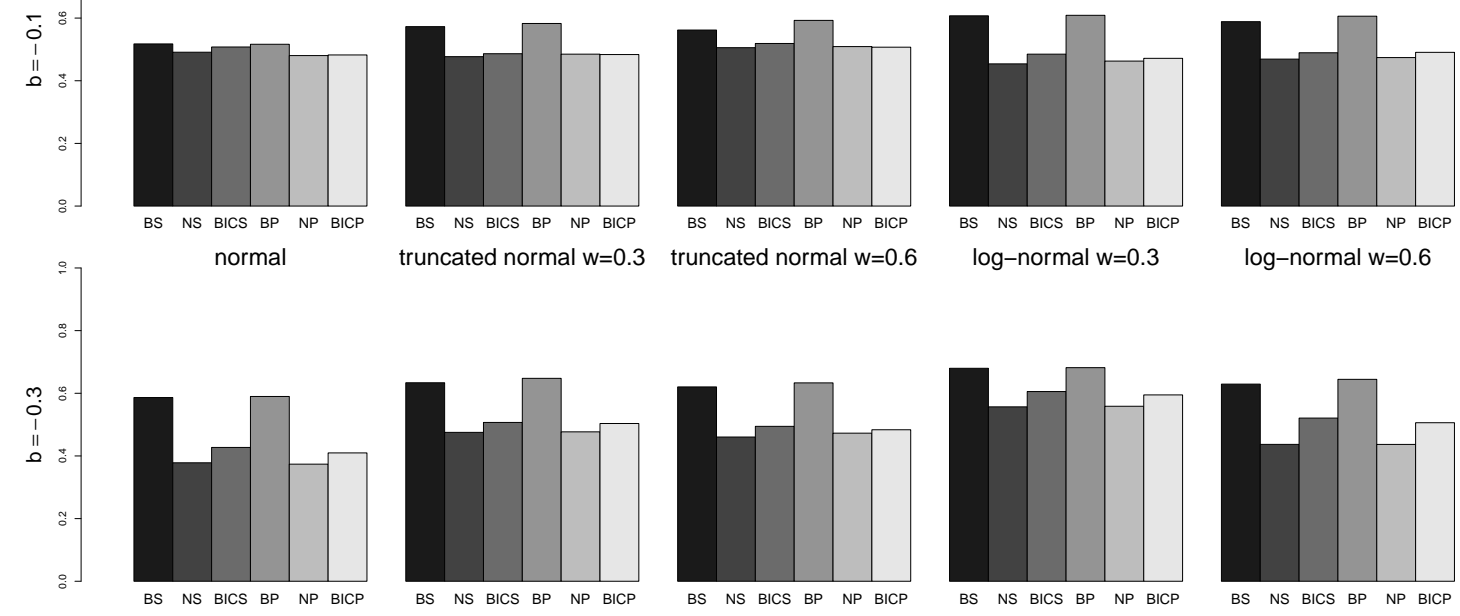

runcated normal $\mathrm{W}=0.3$ truncated normal $\mathrm{w}=0.6$

log-normal $\mathrm{w}=0.3$

log-normal $\mathrm{w}=0.6$
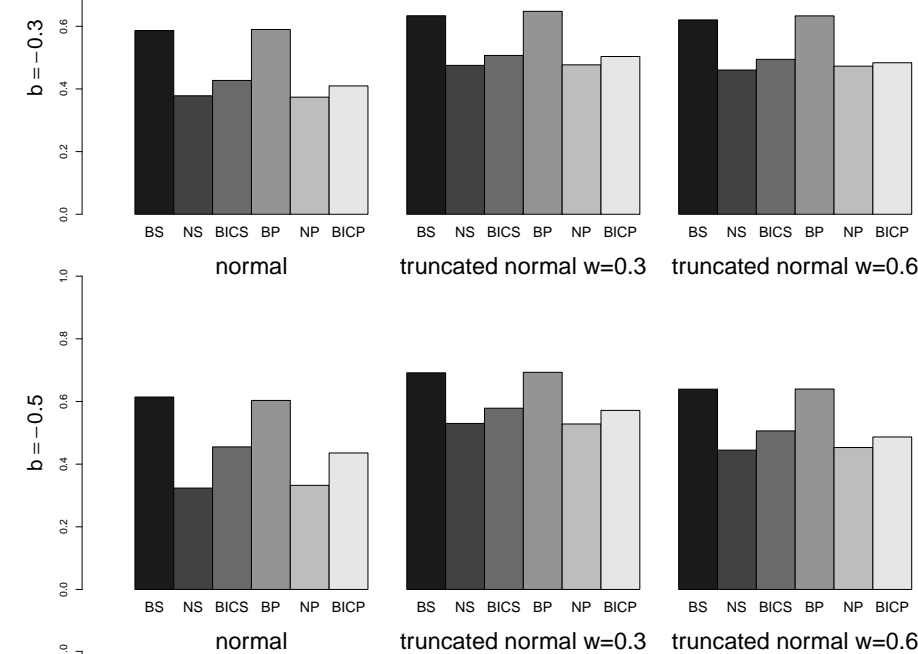

truncated normal $\mathrm{W}=0.3$ truncated normal $\mathrm{w}=0.6$
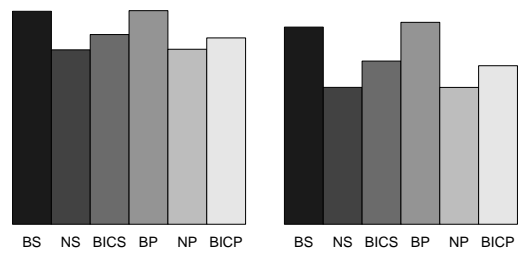

log-normal $\mathrm{w}=0.3$

log-normal $\mathrm{w}=0.6$
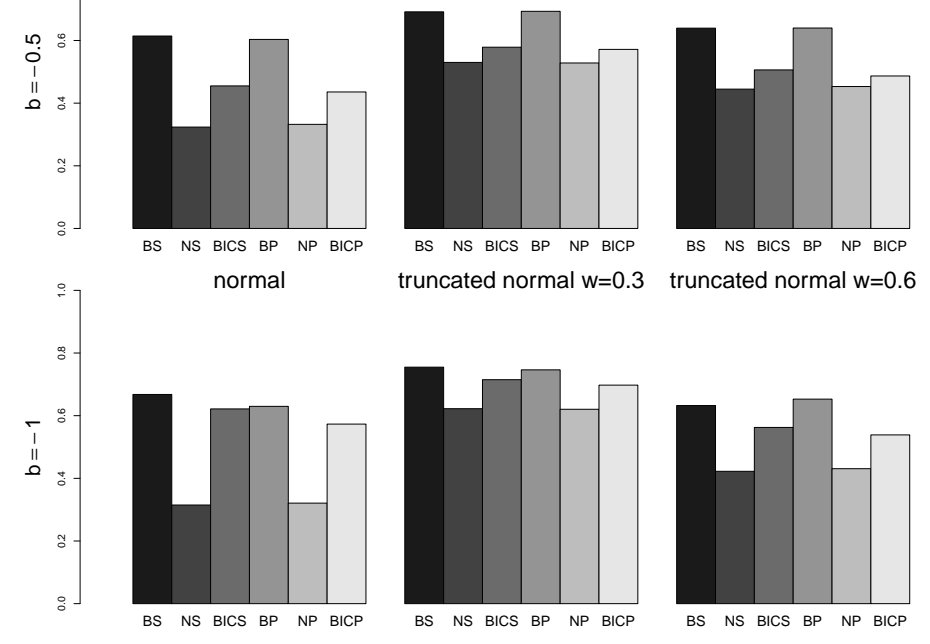

truncated normal $\mathrm{W}=0.3$

truncated normal $\mathrm{W}=0.6$
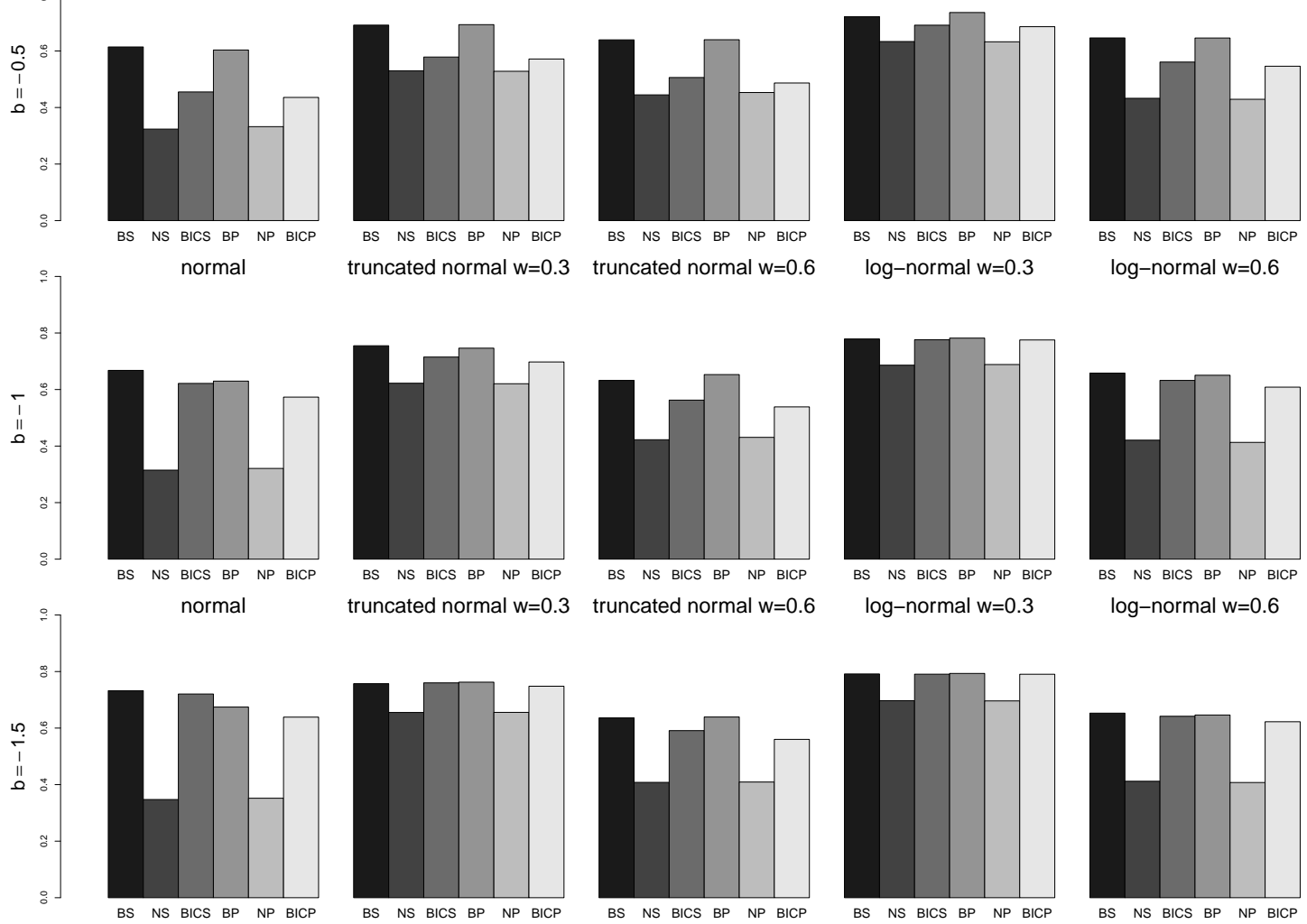

truncated normal $\mathrm{W}=0.3$ truncated normal $\mathrm{W}=0.6$

log-normal $w=0.3$

log-normal $\mathrm{w}=0.6$
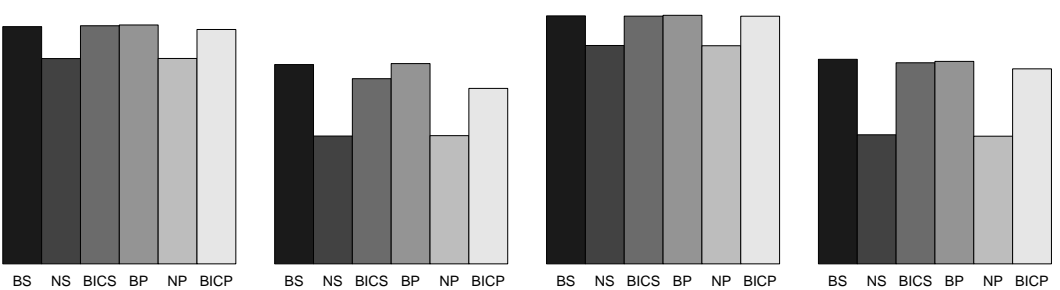

Figure A.2: The probability of Correct MTD Recommendation. The true probablity of toxicity follows an empiric model. $a=-0.5$. The labels BS NS BICS BP NP and BICP for each bar refer to using biomarker model for simple order, non-biomarker model for simple order , BIC model for simple order, biomarker model for partial order, non-biomarker model for partial order and BIC model for partial order. 


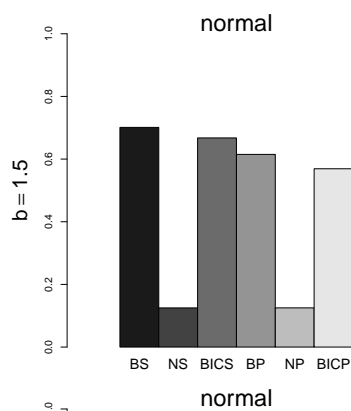

truncated normal $\mathrm{w}=0.3$ truncated normal $\mathrm{w}=0.6$

log-normal $\mathbf{w}=0.3$

log-normal $\mathbf{w}=0.6$
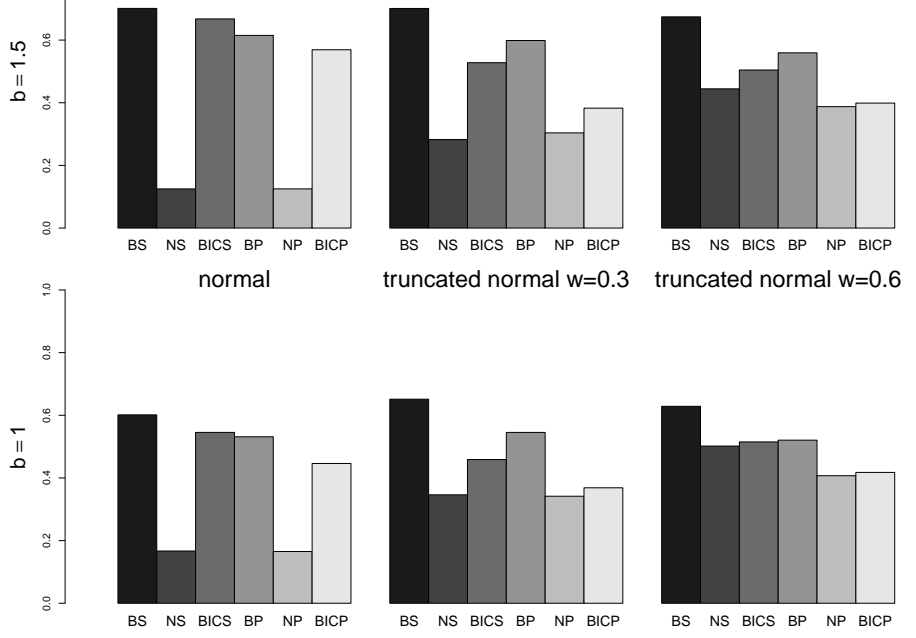

truncated normal $\mathrm{w}=0.3$ truncated normal $\mathrm{w}=0.6$
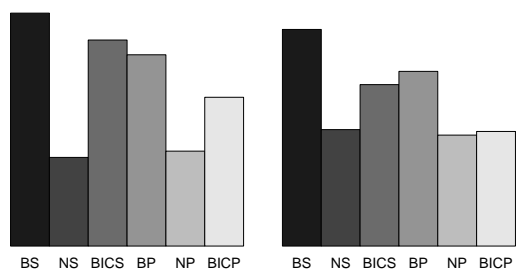

log-normal w=0.3

log-normal $\mathrm{w}=0.6$
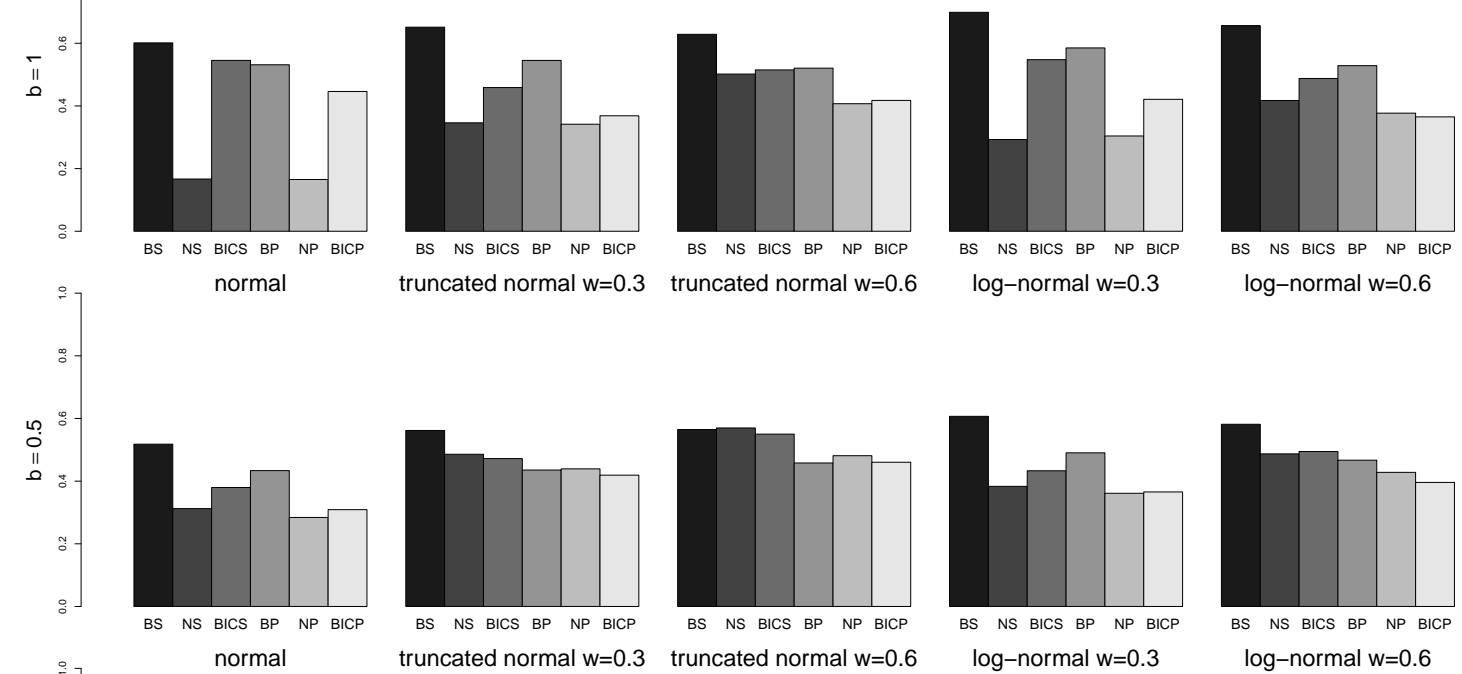

truncated normal $\mathrm{W}=0.3$ truncated normal $\mathrm{w}=0.6$

log-normal $\mathrm{w}=0.3$

log-normal $\mathrm{w}=0.6$
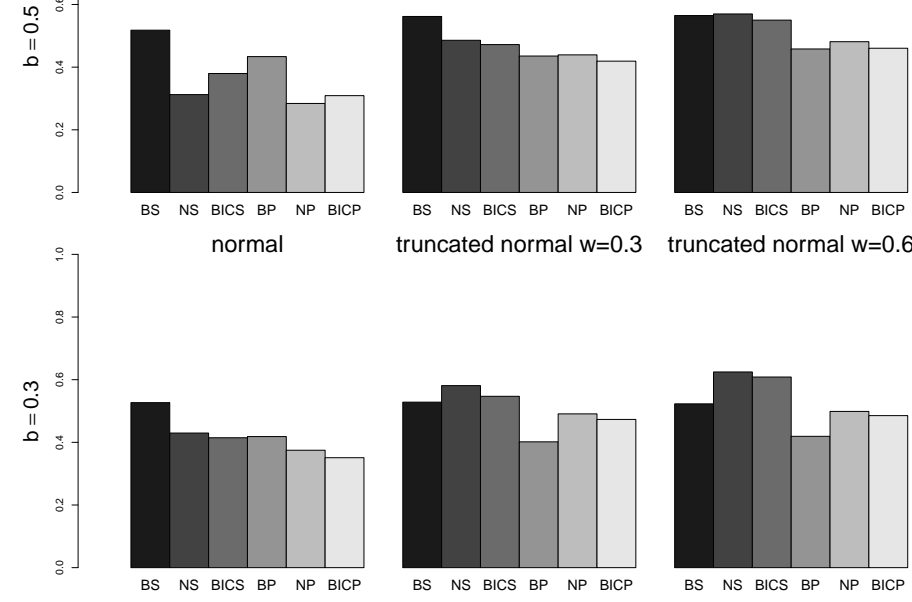

truncated normal $\mathrm{W}=0.3$

truncated normal $\mathrm{W}=0.6$
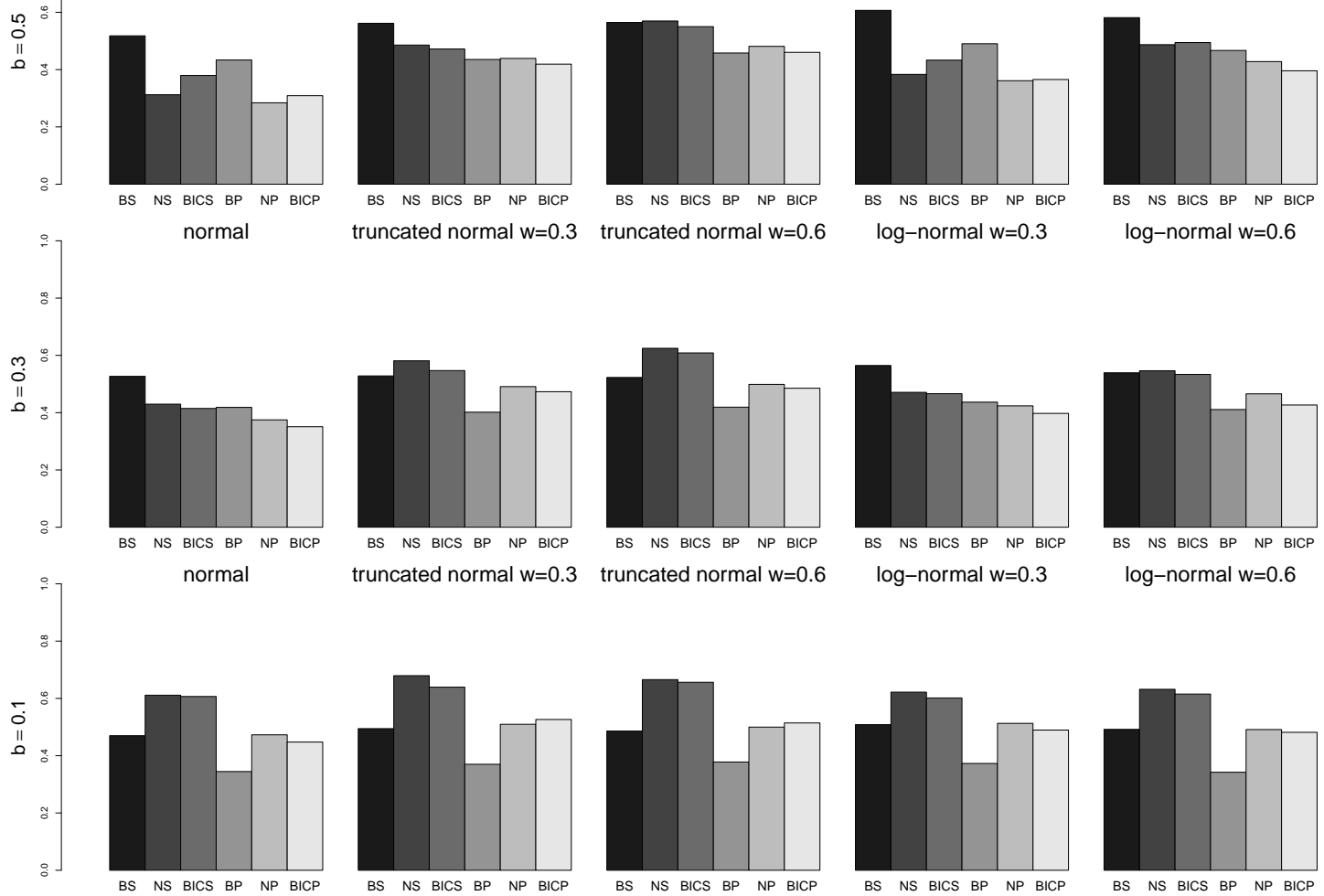

truncated normal $\mathrm{W}=0.3$ truncated normal $\mathrm{W}=0.6$
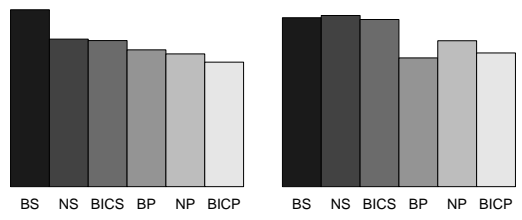

log-normal $\mathrm{w}=0.3$

log-normal $\mathrm{W}=0.6$
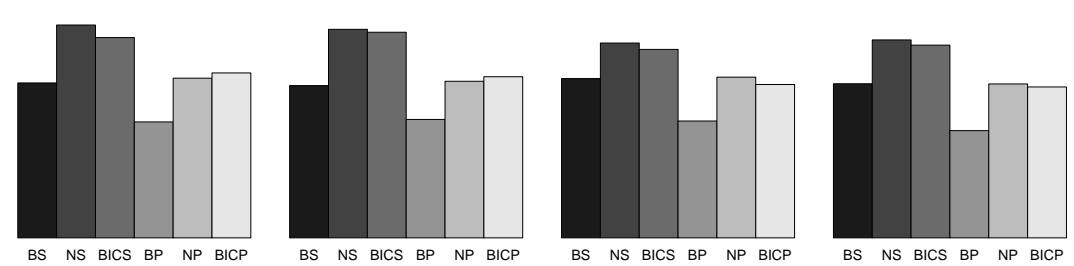

Figure A.3: The probability of Correct MTD Recommendation. The true probablity of toxicity follows an empiric model. $a=0.42$. The labels BS NS BICS BP NP and BICP for each bar refer to using biomarker model for simple order, non-biomarker model for simple order , BIC model for simple order, biomarker model for partial order, non-biomarker model for partial order and BIC model for partial order. 


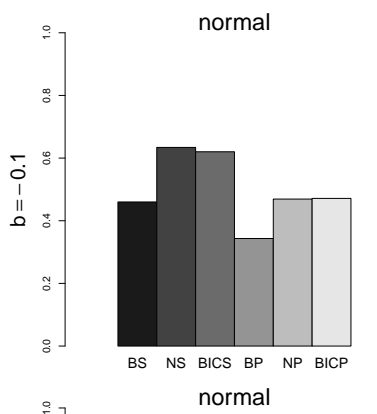

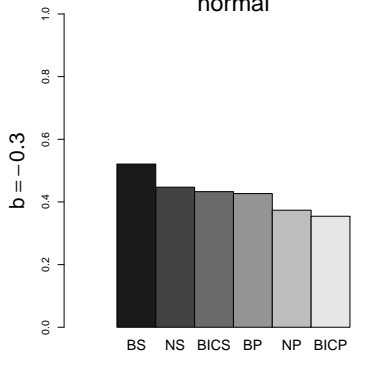
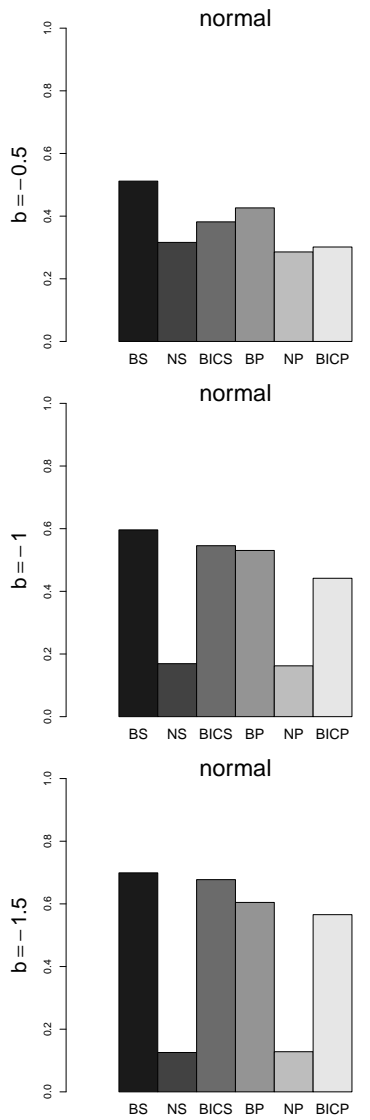

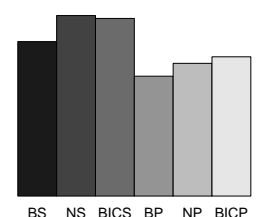

truncated normal $\mathrm{W}=0.3$
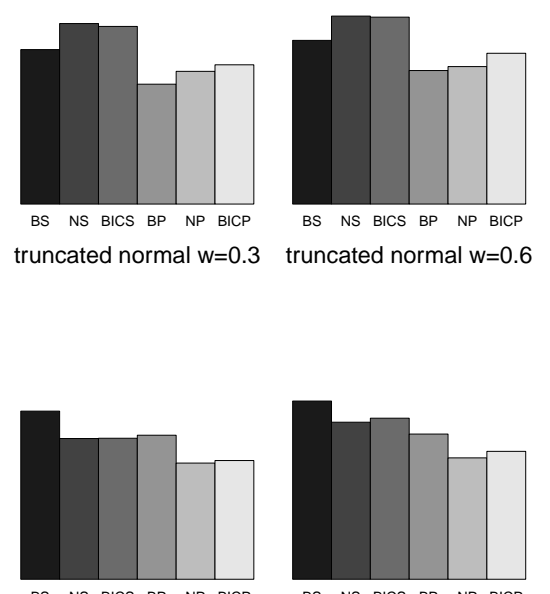

BS NS BICS BP NP BICP truncated normal $\mathrm{w}=0.3$

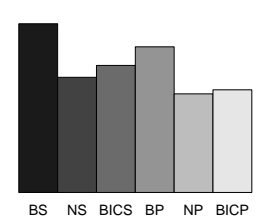

truncated normal $\mathrm{w}=0.3$
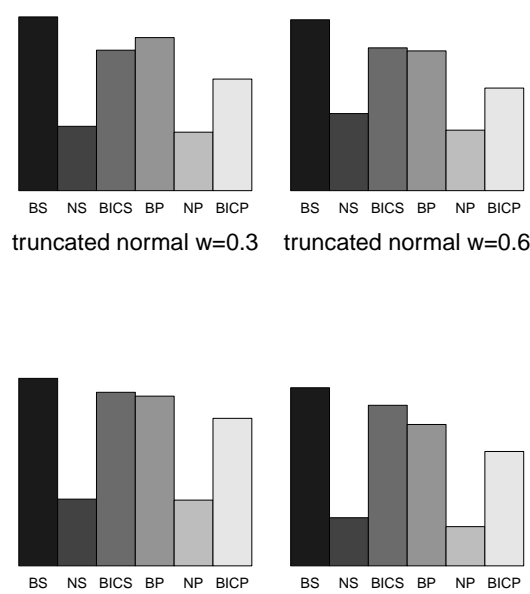

truncated normal $\mathrm{W}=0.6$

truncated normal $\mathrm{w}=0.6$

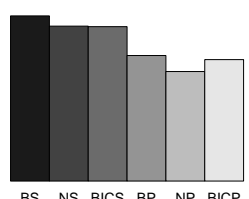

BS NS BICS BP NP BICP log-normal $\mathrm{w}=0.3$

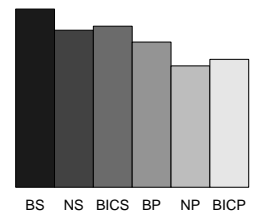

truncated normal $w=0.6$

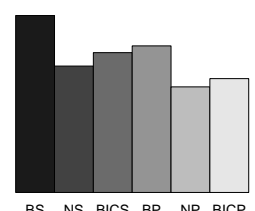

truncated normal $\mathrm{W}=0.6$

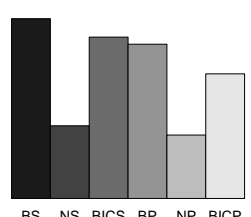

log-normal $\mathrm{w}=0.3$

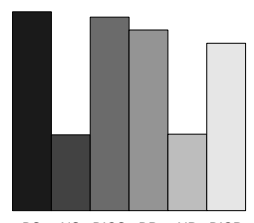

BS NS BICS BP NP BICP log-normal $\mathrm{w}=0.3$

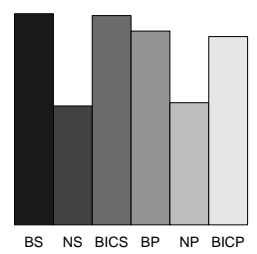

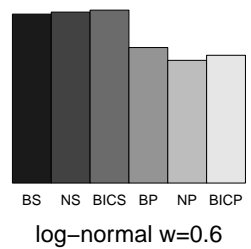

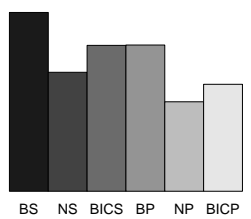

log-normal $\mathrm{w}=0.6$
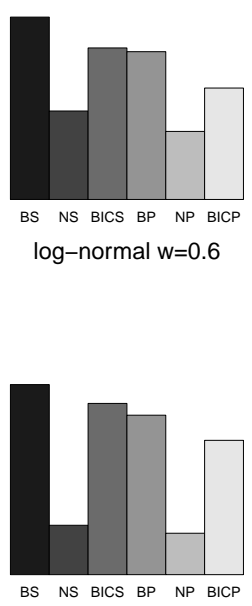

log-normal $\mathrm{w}=0.6$

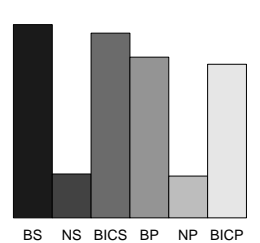

Figure A.4: The probability of Correct MTD Recommendation. The true probablity of toxicity follows an empiric model. $a=0.42$. The labels BS NS BICS BP NP and BICP for each bar refer to using biomarker model for simple order, non-biomarker model for simple order , BIC model for simple order, biomarker model for partial order, non-biomarker model for partial order and BIC model for partial order. 


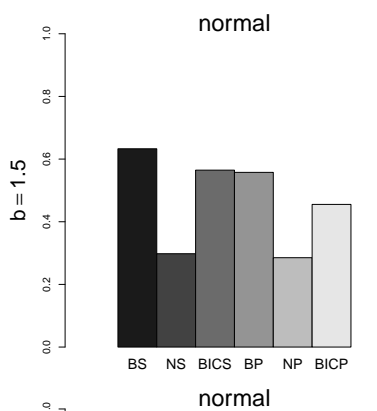

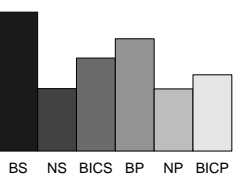

truncated normal $\mathrm{W}=0.3$

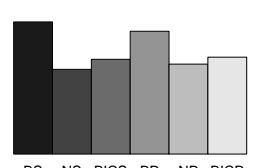

BS NS BICS BP NP BICP truncated normal $\mathrm{w}=0.6$

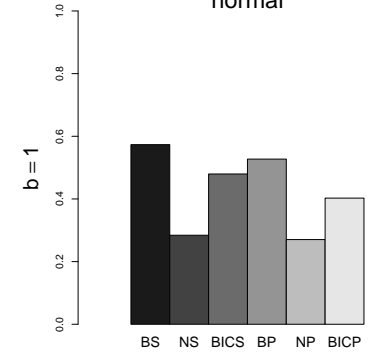

normal

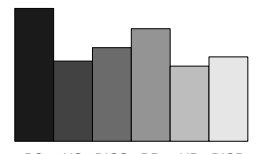

BS NS BICS BP NP BICP truncated normal $\mathrm{w}=0.3$

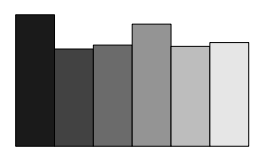

truncated normal $W=0.6$

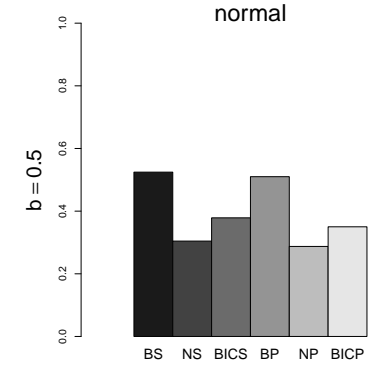

normal

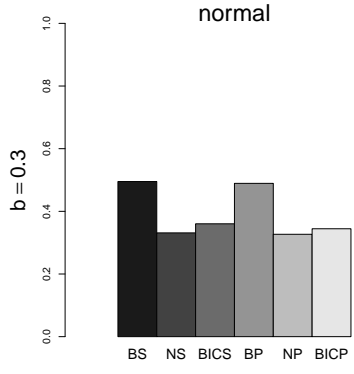

normal
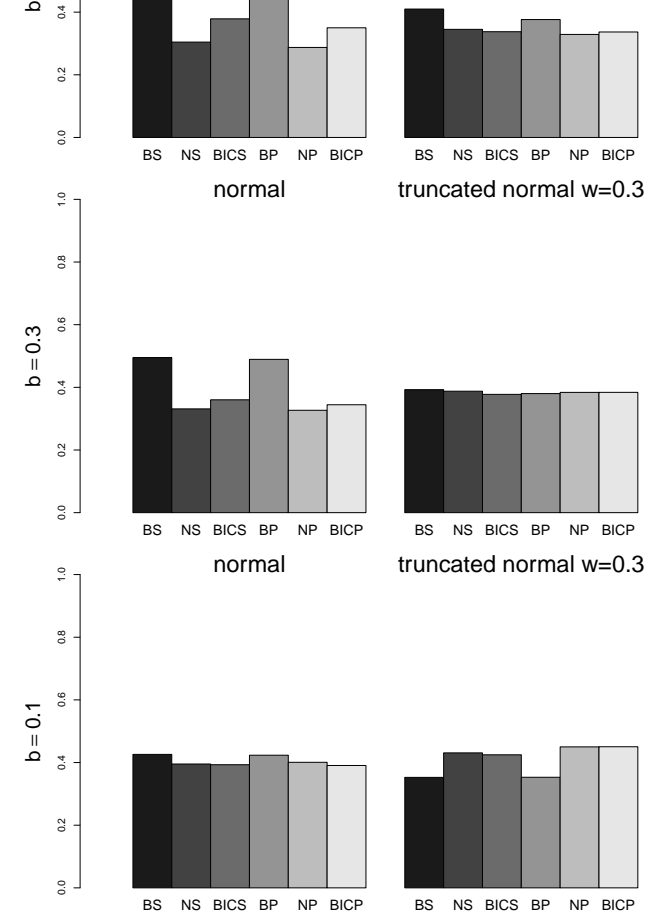

BS NS BICS BP NP BICP truncated normal $\mathrm{w}=0.3$

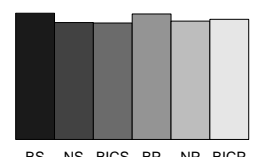

truncated normal $\mathrm{w}=0.6$
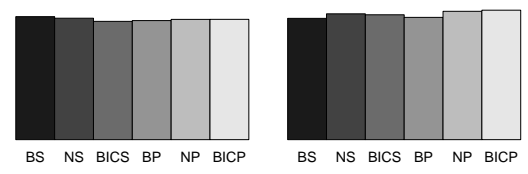

truncated normal $\mathrm{w}=0.3$ truncated normal $\mathrm{w}=0.6$
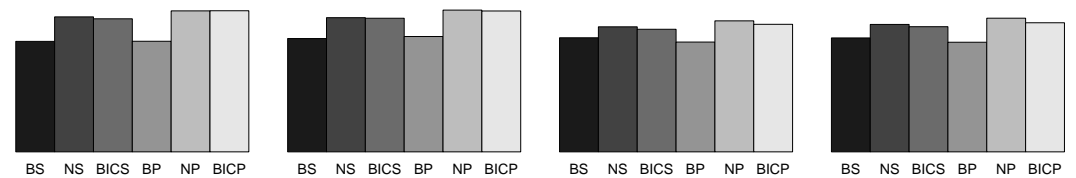

Figure A.5: The percentage of patients treated at the MTDs. The true probablity of toxicity follows an empiric model. $a=-0.5$. The labels BS NS BICS BP NP and BICP for each bar refer to using biomarker model for simple order, non-biomarker model for simple order , BIC model for simple order, biomarker model for partial order, non-biomarker model for partial order and BIC model for partial order. 

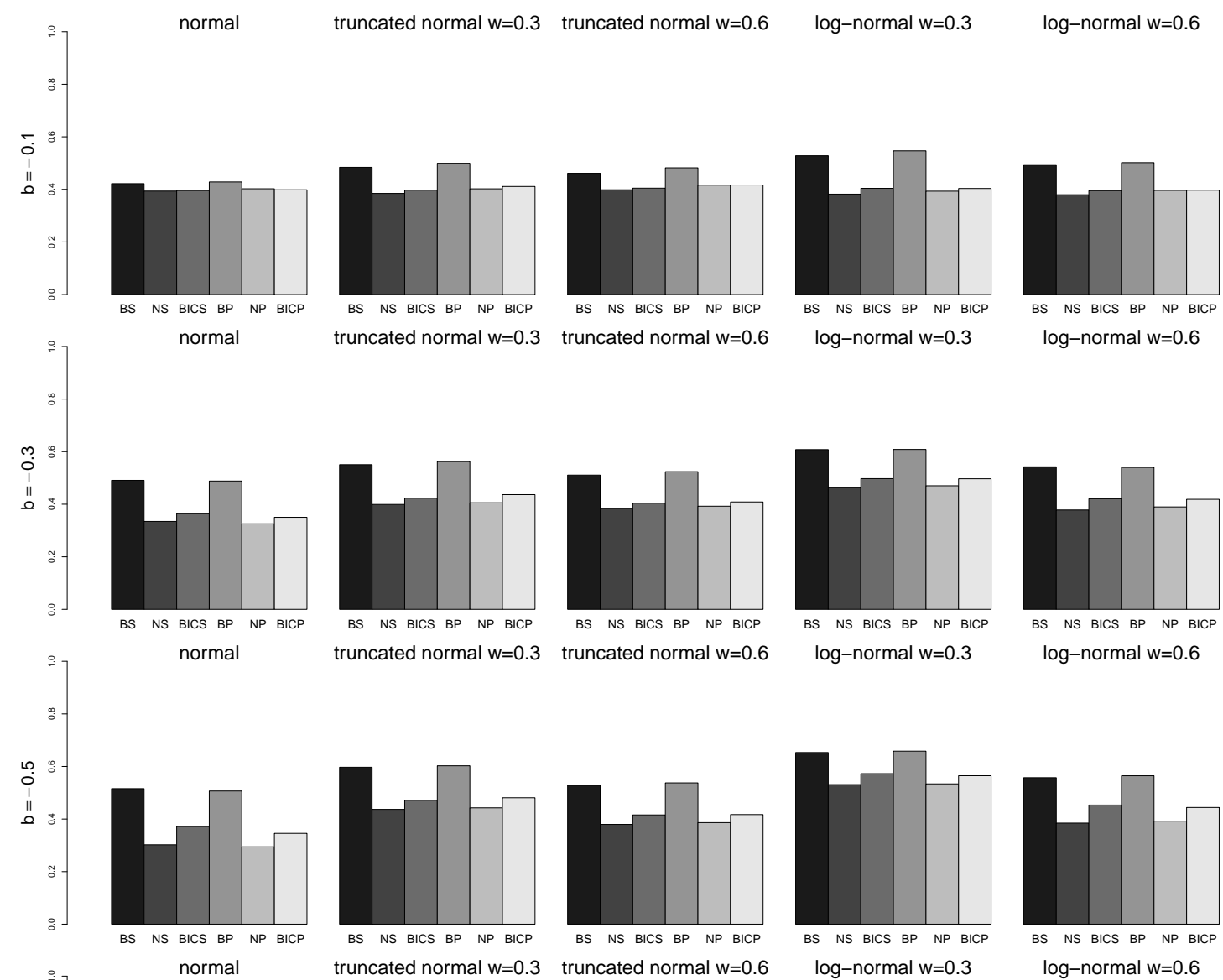

truncated normal $\mathrm{W}=0.3$ truncated normal $\mathrm{W}=0.6$
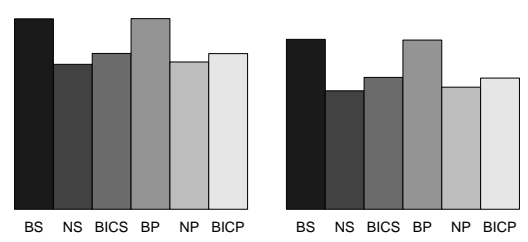

log-normal $w=0.3$

log-normal $\mathrm{W}=0.6$
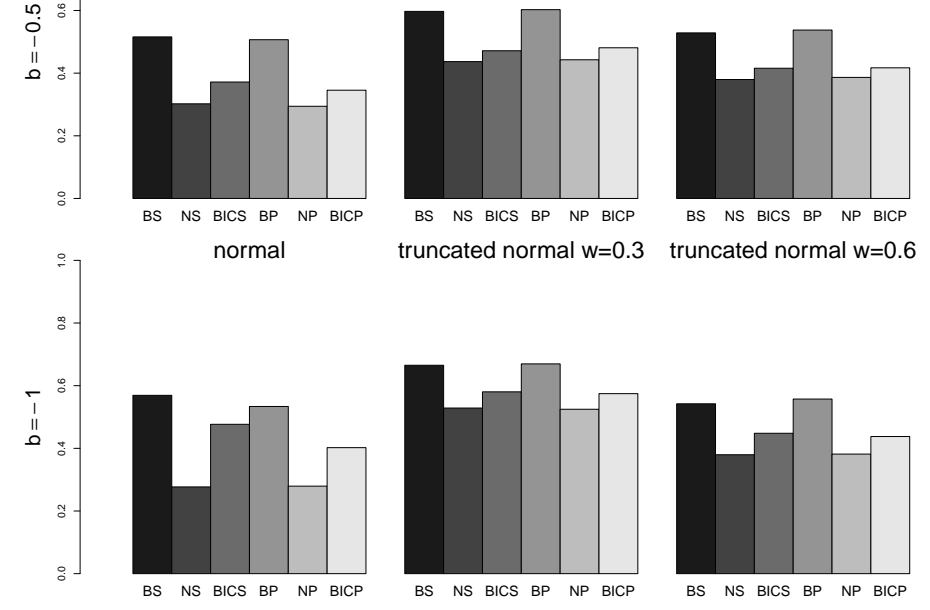

truncated normal $\mathrm{w}=0.3$

truncated normal $\mathrm{W}=0.6$
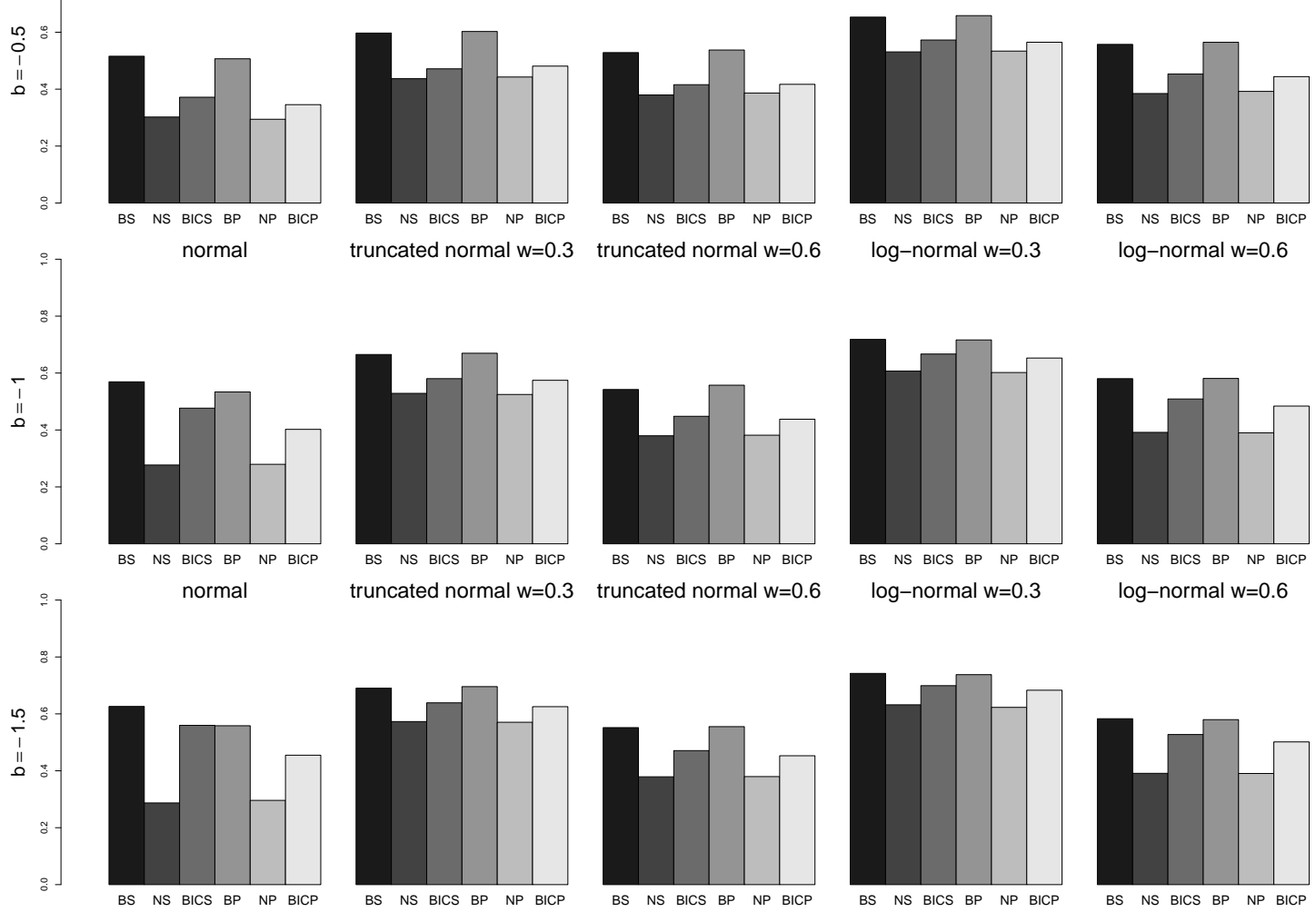
truncated normal $\mathrm{w}=0.3$ truncated normal $\mathrm{w}=0.6$

log-normal $\mathrm{w}=0.3$
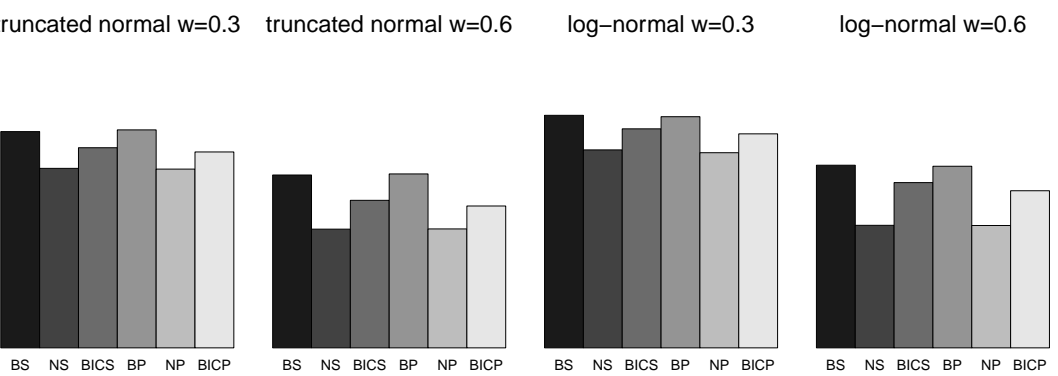

Figure A.6: The percentage of patients treated at the MTDs. The true probablity of toxicity follows an empiric model. $a=-0.5$. The labels BS NS BICS BP NP and BICP for each bar refer to using biomarker model for simple order, non-biomarker model for simple order , BIC model for simple order, biomarker model for partial order, non-biomarker model for partial order and BIC model for partial order. 

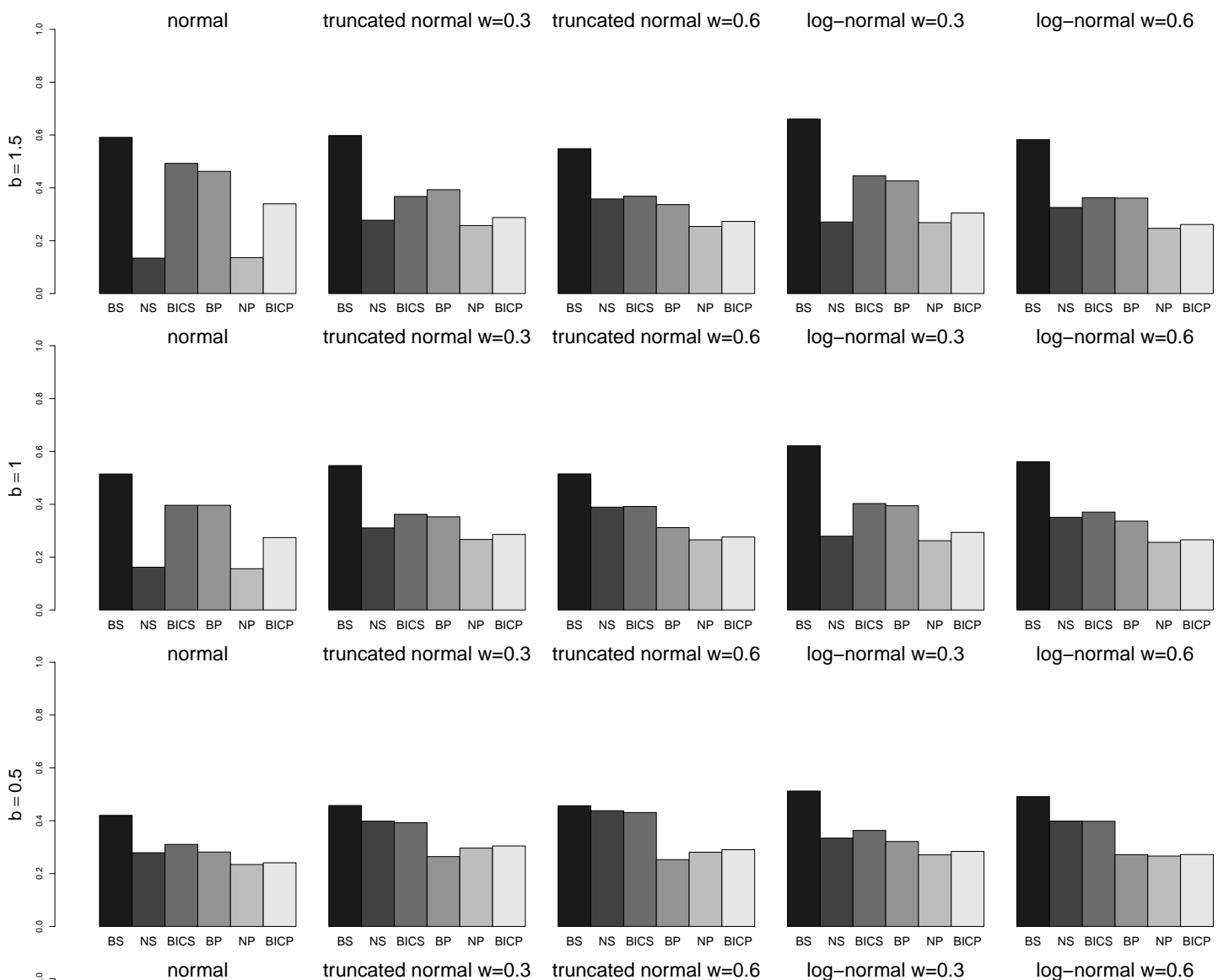

truncated normal $\mathrm{w}=0.3$ truncated normal $\mathrm{w}=0.6$
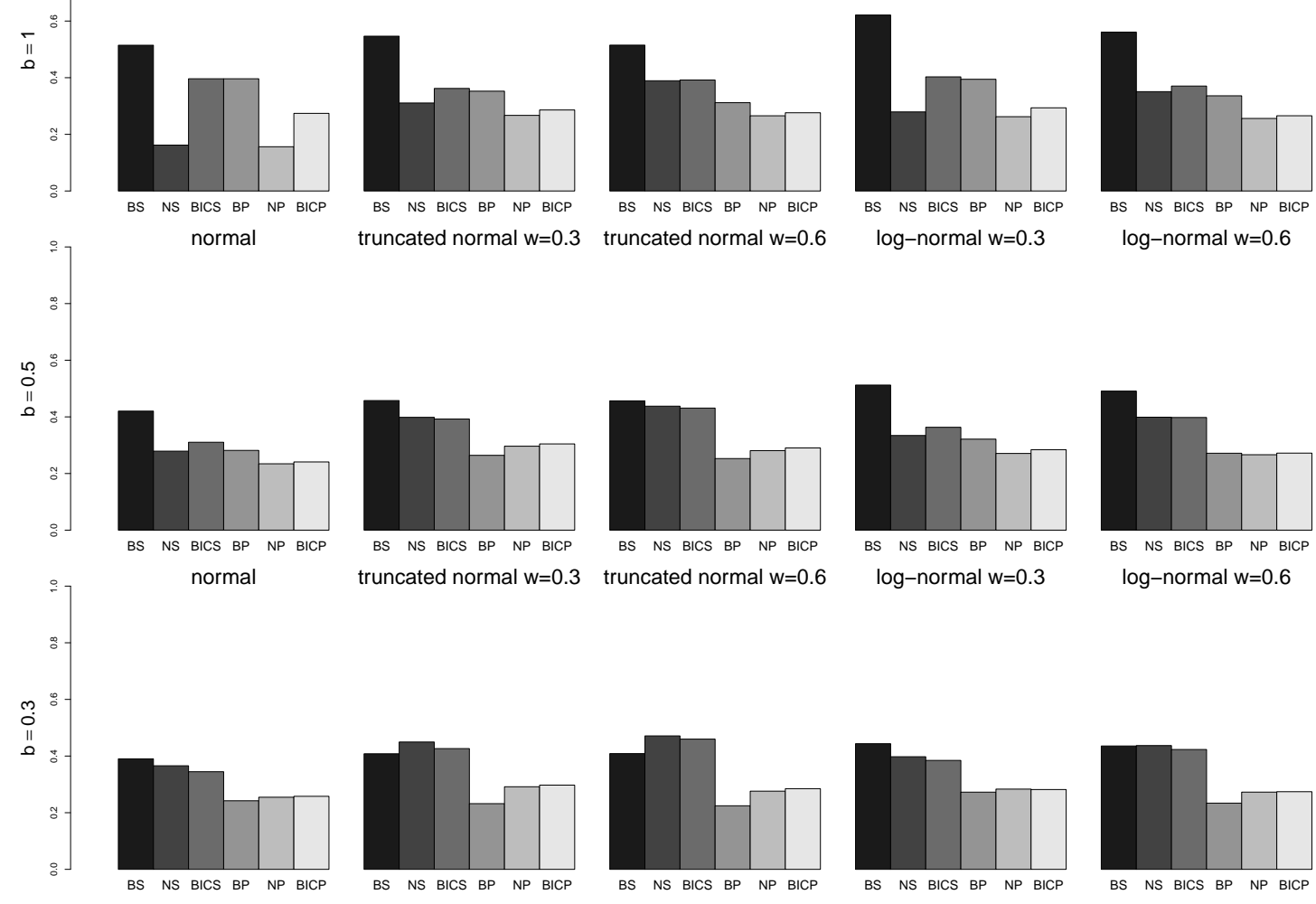

truncated normal $\mathrm{w}=0.3$

truncated normal $\mathrm{W}=0.6$
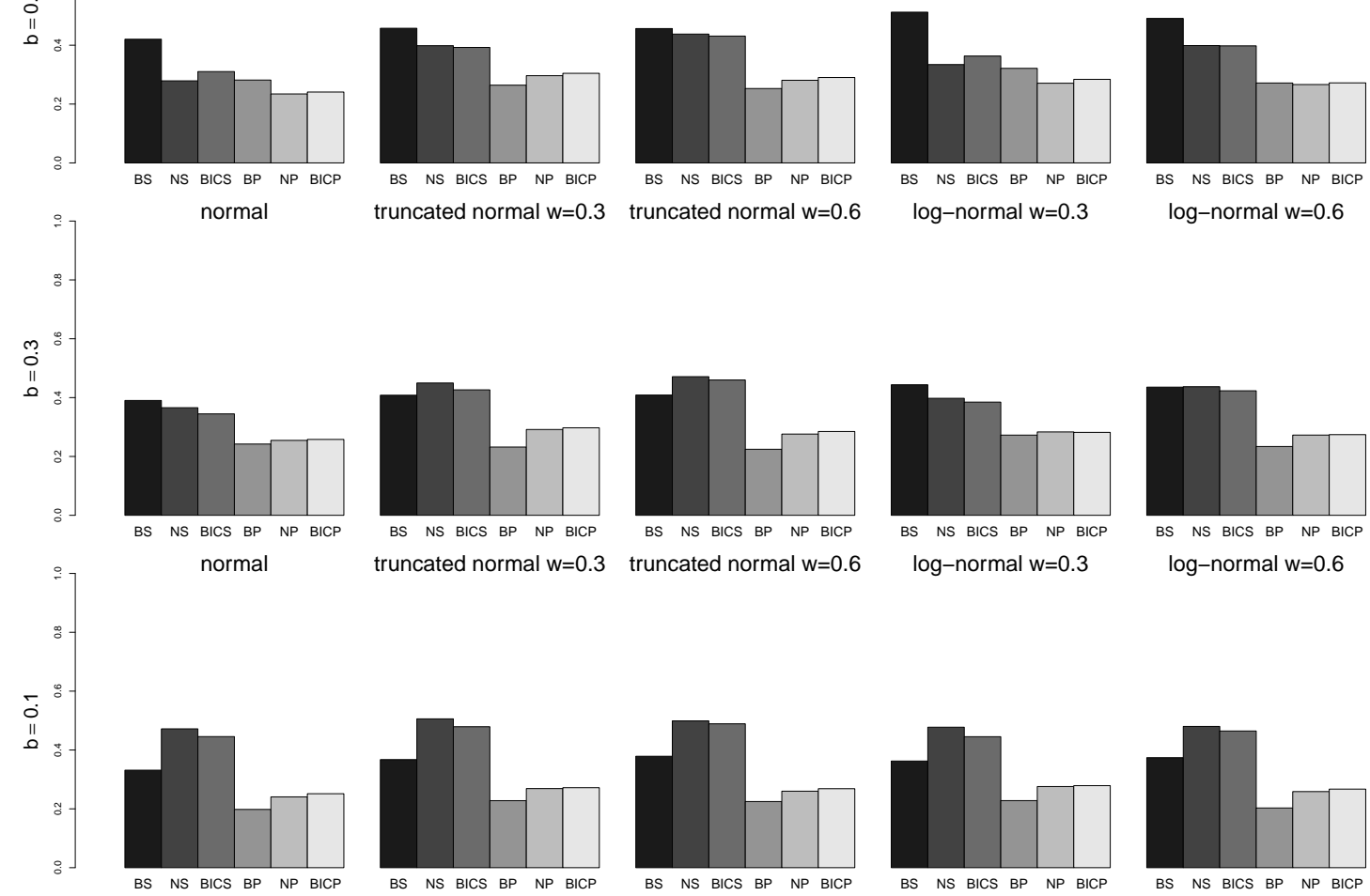
truncated normal $\mathrm{W}=0.3$ truncated normal $\mathrm{W}=0.6$

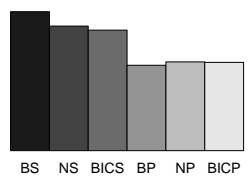

log-normal $\mathrm{w}=0.3$
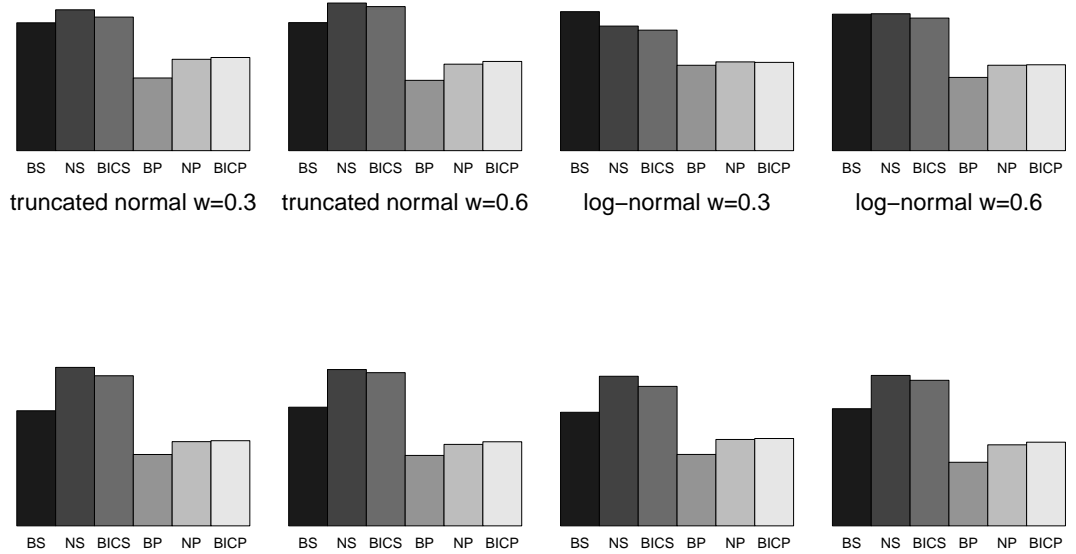

Figure A.7: The percentage of patients treated at the MTDs. The true probablity of toxicity follows an empiric model. $a=0.42$. The labels BS NS BICS BP NP and BICP for each bar refer to using biomarker model for simple order, non-biomarker model for simple order , BIC model for simple order, biomarker model for partial order, non-biomarker model for partial order and BIC model for partial order. 


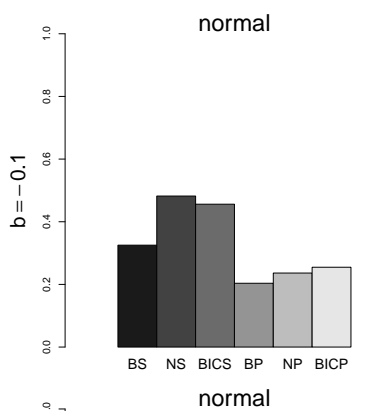

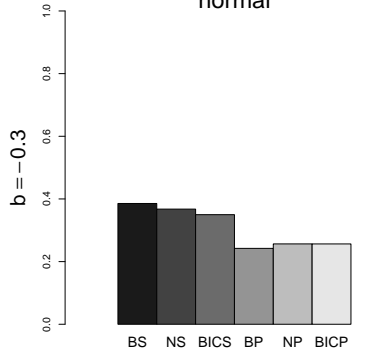

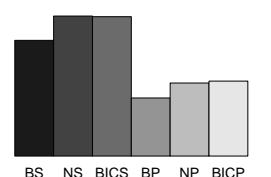

truncated normal $\mathrm{W}=0.3$

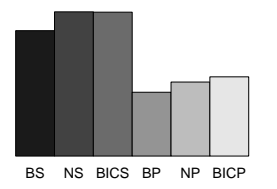

truncated normal $\mathrm{w}=0.6$

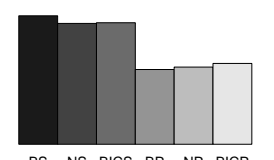

BS NS BICS BP NP BICP

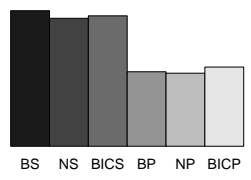

truncated normal $\mathrm{w}=0.6$

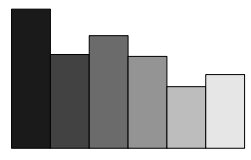

BS NS BICS BP NP BICP

log-normal w=0.3
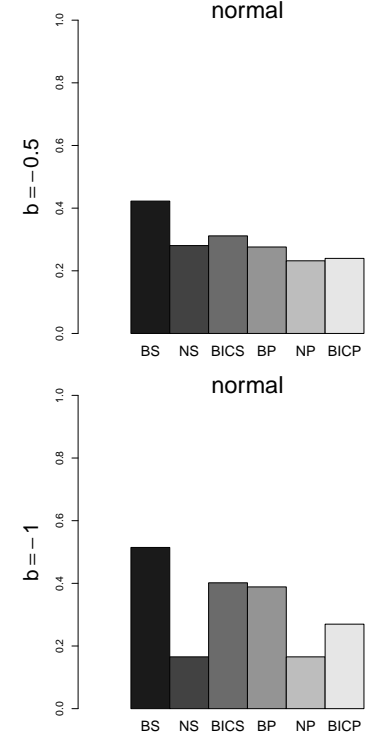

normal
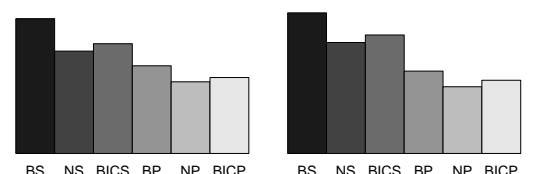

truncated normal $\mathrm{w}=0.3$

truncated normal $\mathrm{W}=0.6$

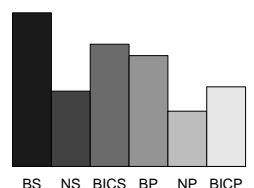

log-normal $w=0.3$
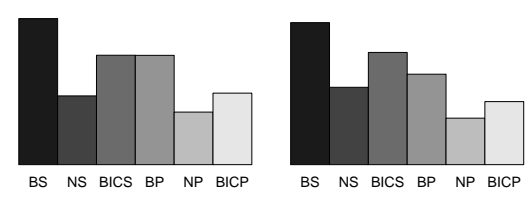

truncated normal $\mathrm{w}=0.6$

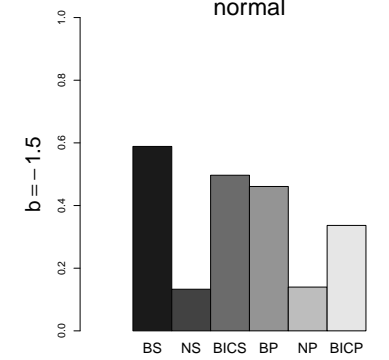

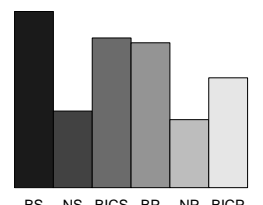

BS NS BICS BP NP BIC
log-normal $W=0.3$

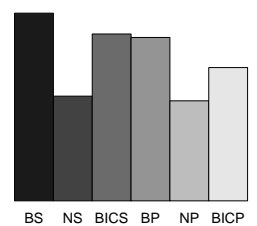

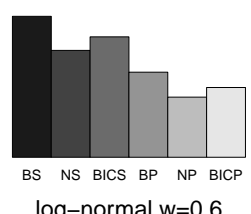
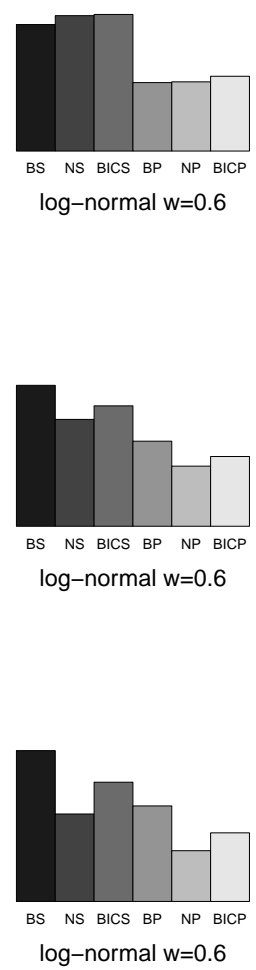

log-normal $\mathrm{w}=0.6$

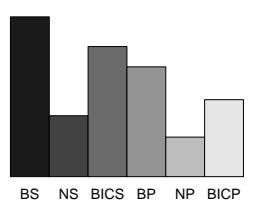

log-normal w=0.6

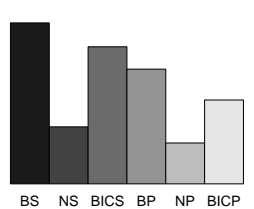

Figure A.8: The percentage of patients treated at the MTDs. The true probablity of toxicity follows an empiric model. $a=0.42$. The labels BS NS BICS BP NP and BICP for each bar refer to using biomarker model for simple order, non-biomarker model for simple order , BIC model for simple order, biomarker model for partial order, non-biomarker model for partial order and BIC model for partial order. 


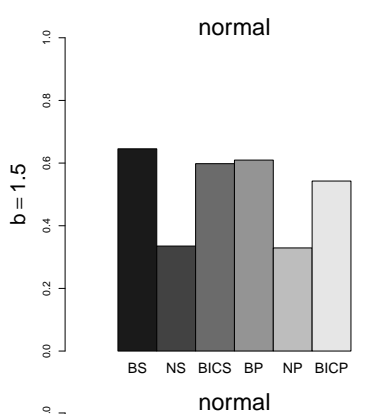

truncated normal $\mathrm{w}=0.3$ truncated normal $\mathrm{w}=0.6$

log-normal w=0.3

log-normal $\mathbf{w}=0.6$

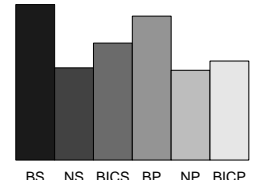

truncated normal $w=0.3$

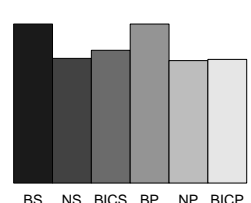

NS BICS BP NP BICP truncated normal $\mathrm{W}=0.6$

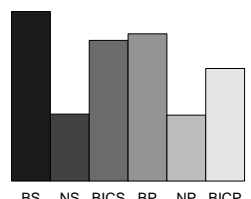

BS NS BICS BP NP BICP log-normal $\mathrm{w}=0.3$

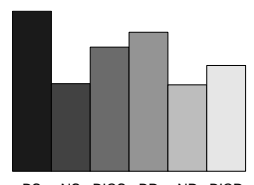

BS NS BICS BP NP BICP log-normal $\mathrm{w}=0.3$

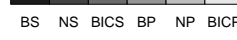

truncated normal $w=0.6$

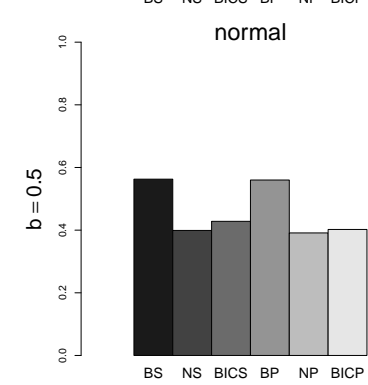

normal
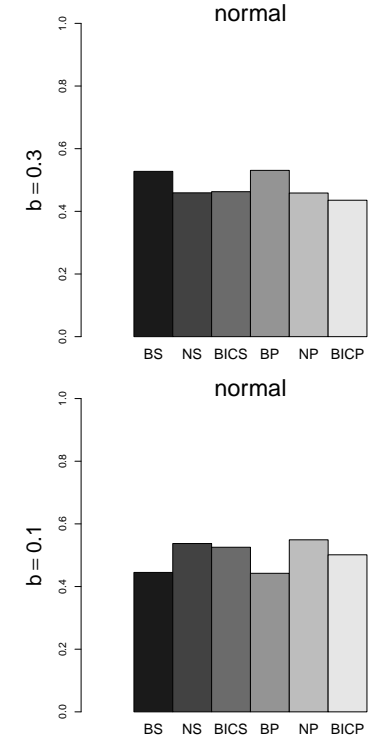

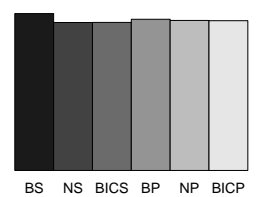

truncated normal $\mathrm{w}=0.3$

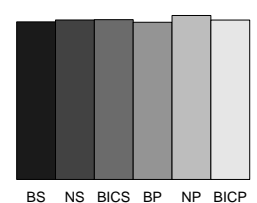

truncated normal $\mathrm{W}=0.6$

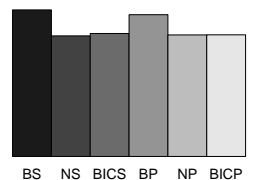

log-normal $\mathrm{w}=0.3$
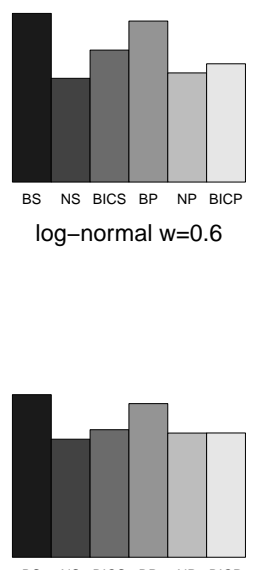

BS NS BICS BP NP BICP log-normal $\mathrm{w}=0.6$
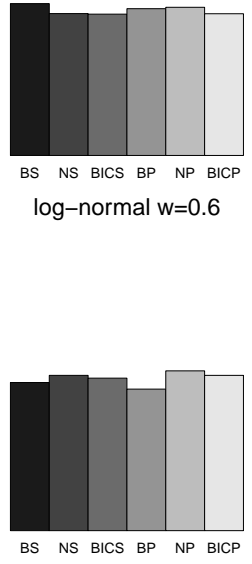

log-normal $\mathrm{w}=0.6$

BS NS BICS BP NP BICP log-normal $\mathrm{w}=0.3$ truncated normal $\mathrm{W}=0.3$ truncated normal $\mathrm{W}=0.6$
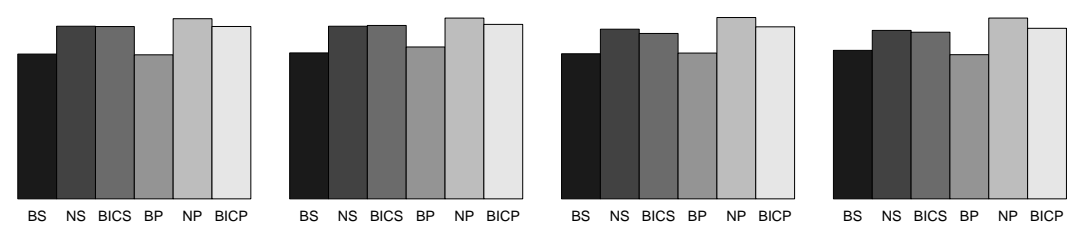

Figure A.9: The probability of Correct MTD Recommendation. The true probablity of toxicity follows a logit model. $a_{i}$ is in scenario 1 . The labels BS NS BICS BP $\mathrm{NP}$ and BICP for each bar refer to using biomarker model for simple order , nonbiomarker model for simple order, BIC model for simple order, biomarker model for partial order, non-biomarker model for partial order and BIC model for partial order. 


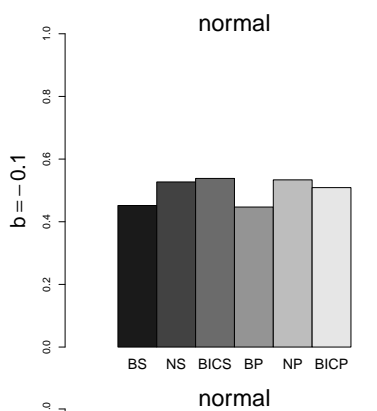

truncated normal $\mathrm{w}=0.3$ truncated normal $\mathrm{w}=0.6$

log-normal w=0.3

log-normal $\mathbf{w}=0.6$
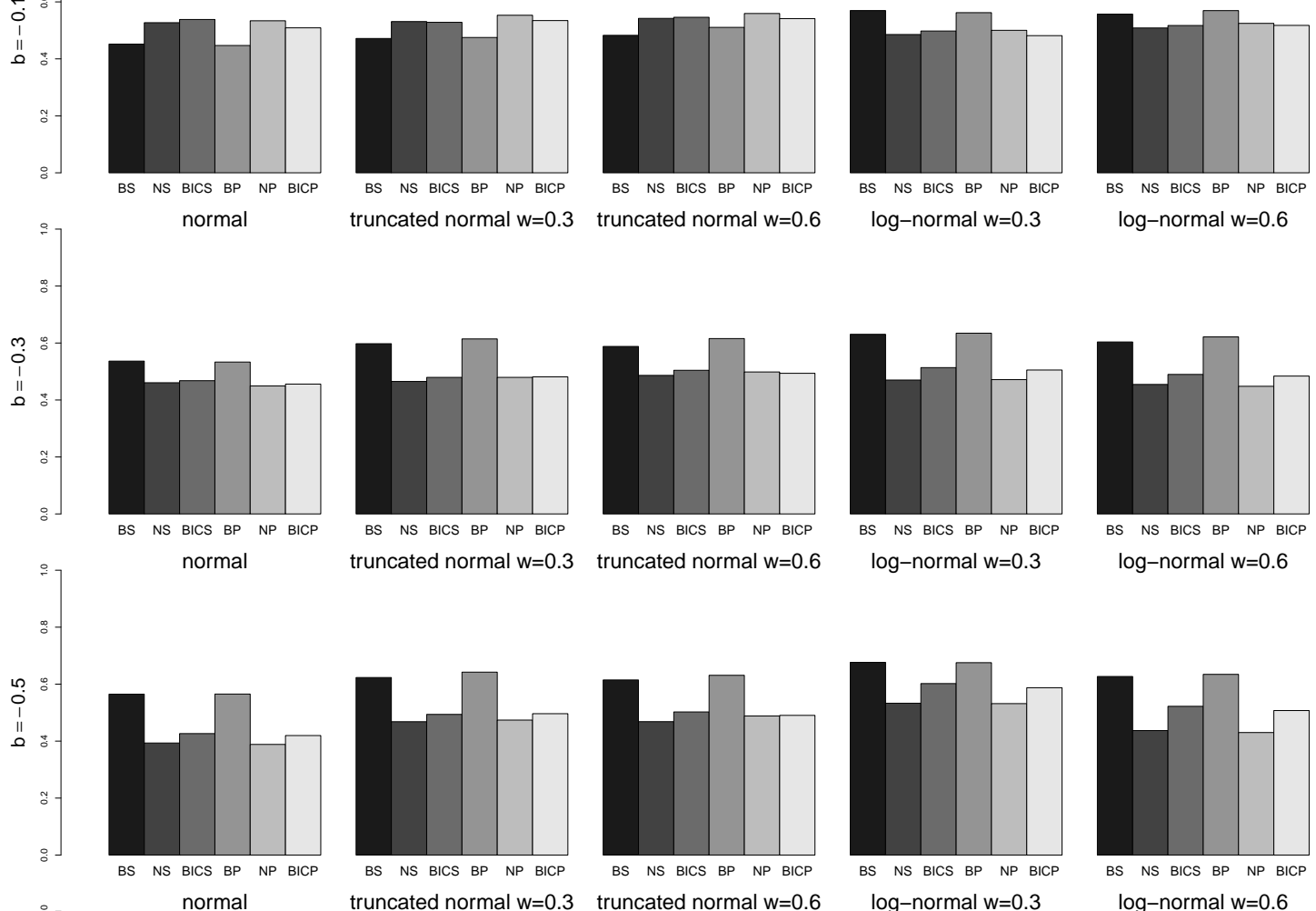

truncated normal $\mathrm{w}=0.3$ truncated normal $\mathrm{w}=0.6$

log-normal $\mathrm{w}=0.3$

log-normal $\mathrm{w}=0.6$
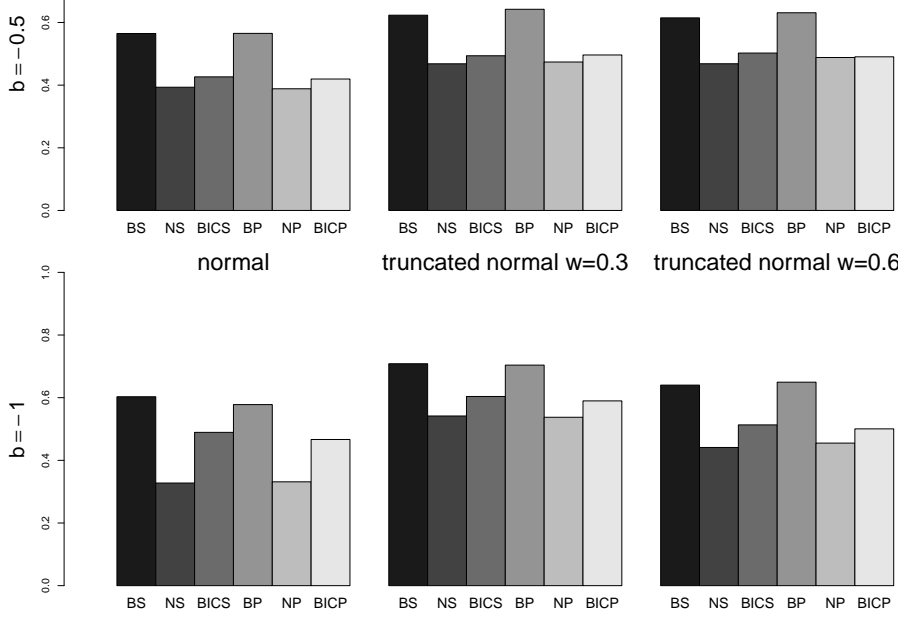

truncated normal $\mathrm{W}=0.3$

truncated normal $\mathrm{w}=0.6$
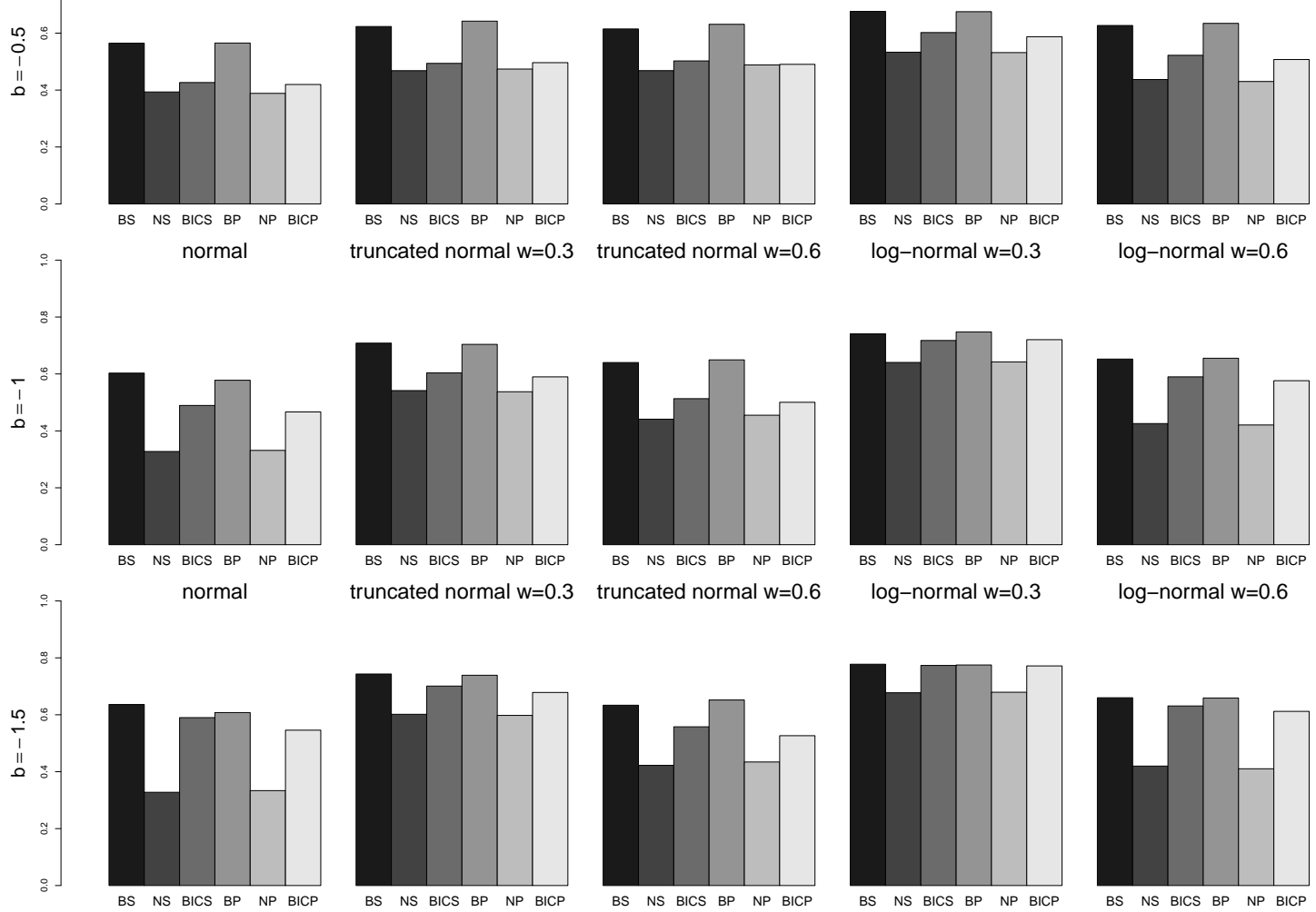

truncated normal $\mathrm{W}=0.3$ truncated normal $\mathrm{W}=0.6$

log-normal $\mathrm{w}=0.3$

log-normal $\mathrm{W}=0.6$
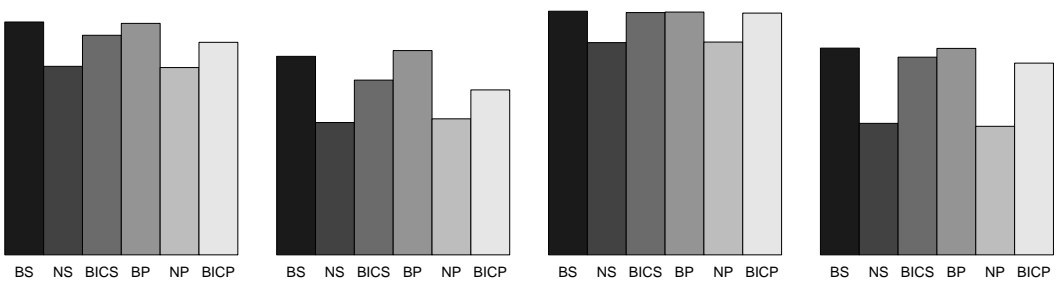

Figure A.10: The probability of Correct MTD Recommendation. The true probablity of toxicity follows a logit model. $a_{i}$ is in scenario 1. The labels BS NS BICS BP NP and BICP for each bar refer to using biomarker model for simple order , non-biomarker model for simple order , BIC model for simple order, biomarker model for partial order, non-biomarker model for partial order and BIC model for partial order. 


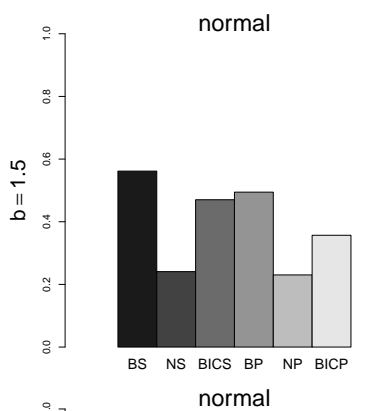

truncated normal $\mathrm{w}=0.3$ truncated normal $w=0.6$

log-normal $\mathrm{w}=0.3$

log-normal $\mathbf{w}=0.6$
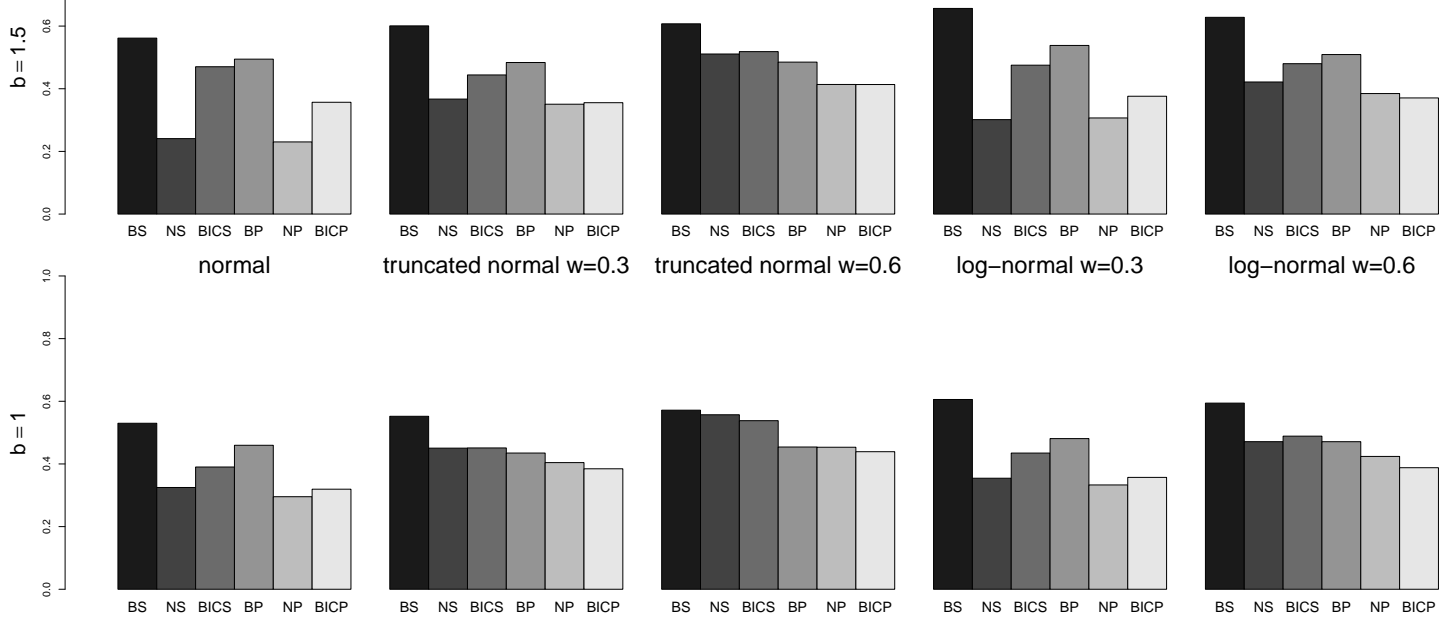

truncated normal $\mathrm{w}=0.3$ truncated normal $\mathrm{w}=0.6$

log-normal $\mathrm{W}=0.3$

log-normal $w=0.6$
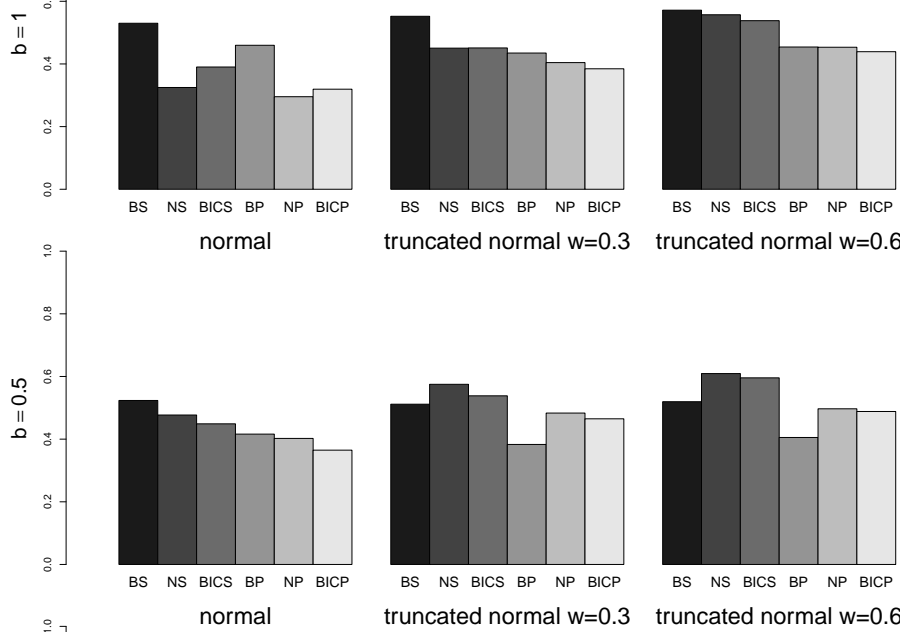

truncated normal $\mathrm{w}=0.3$ truncated normal $\mathrm{w}=0.6$
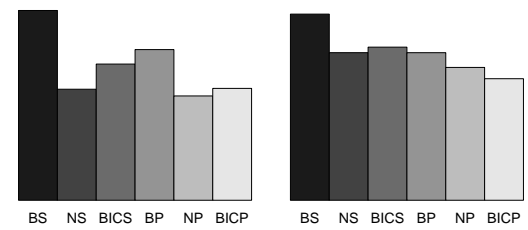

log-normal $\mathrm{w}=0.3$

log-normal $\mathrm{w}=0.6$
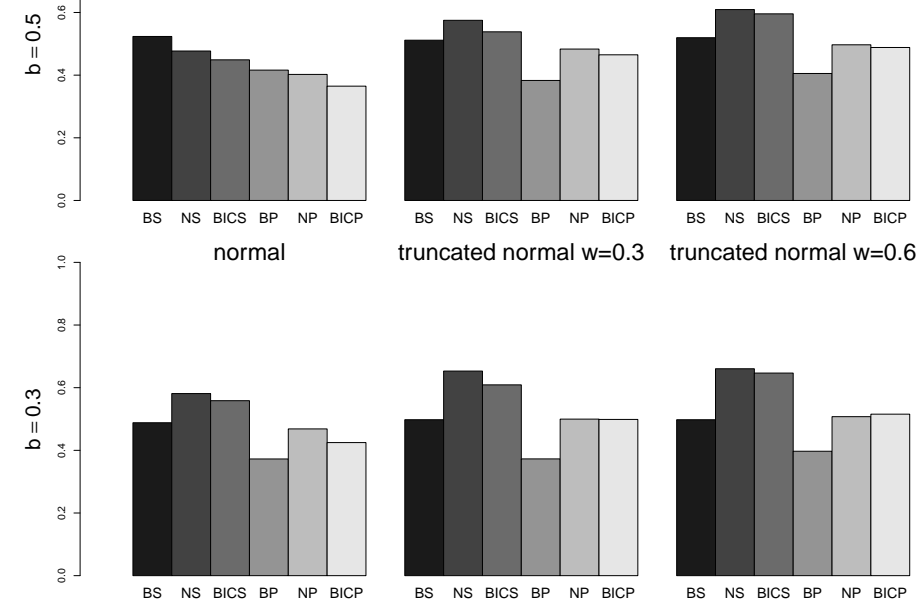

truncated normal $\mathrm{W}=0.3$

truncated normal $\mathrm{W}=0.6$
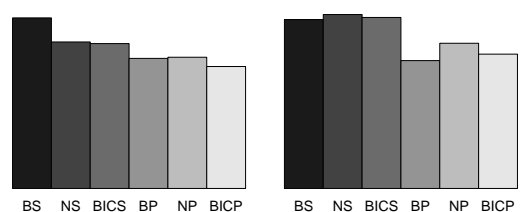

log-normal $\mathrm{w}=0.3$

log-normal $\mathbf{w}=0.6$
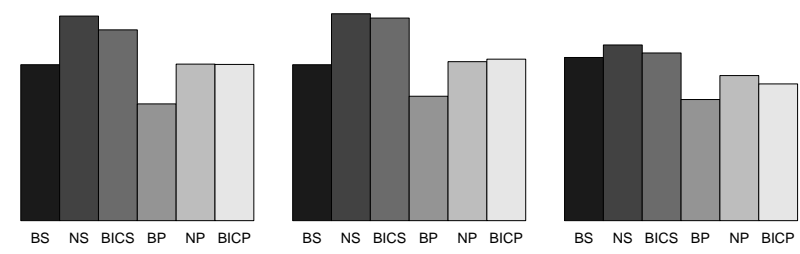

log-normal $w=0.3$

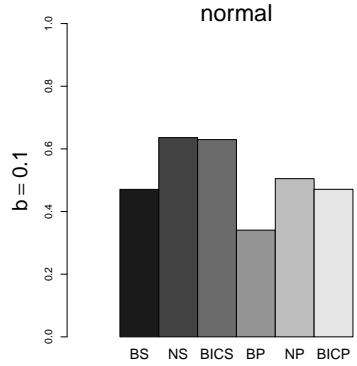

truncated normal $\mathrm{W}=0.3$ truncated normal $\mathrm{w}=0.6$
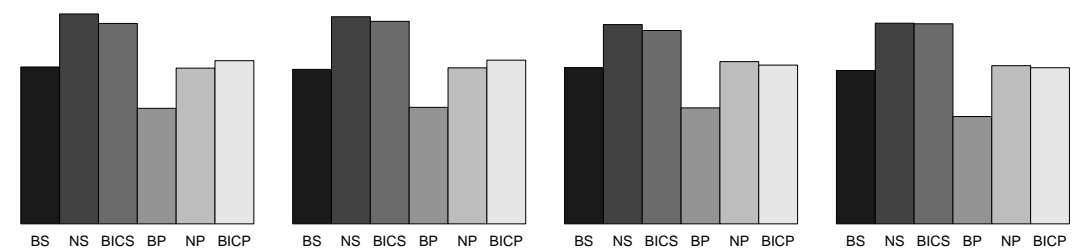

Figure A.11: The probability of Correct MTD Recommendation. The true probablity of toxicity follows a logit model. $a_{i}$ is in scenario 2. The labels BS NS BICS BP NP and BICP for each bar refer to using biomarker model for simple order , non-biomarker model for simple order , BIC model for simple order, biomarker model for partial order, non-biomarker model for partial order and BIC model for partial order. 


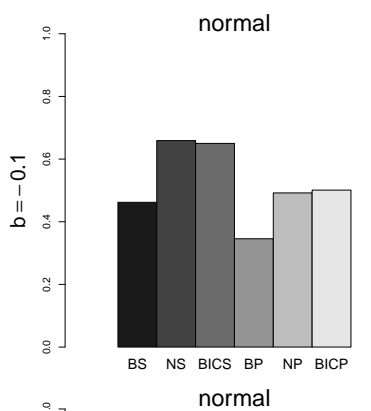

truncated normal $\mathrm{w}=0.3 \quad$ truncated normal $\mathrm{w}=0.6$

log-normal w=0.3

log-normal $\mathbf{w}=0.6$
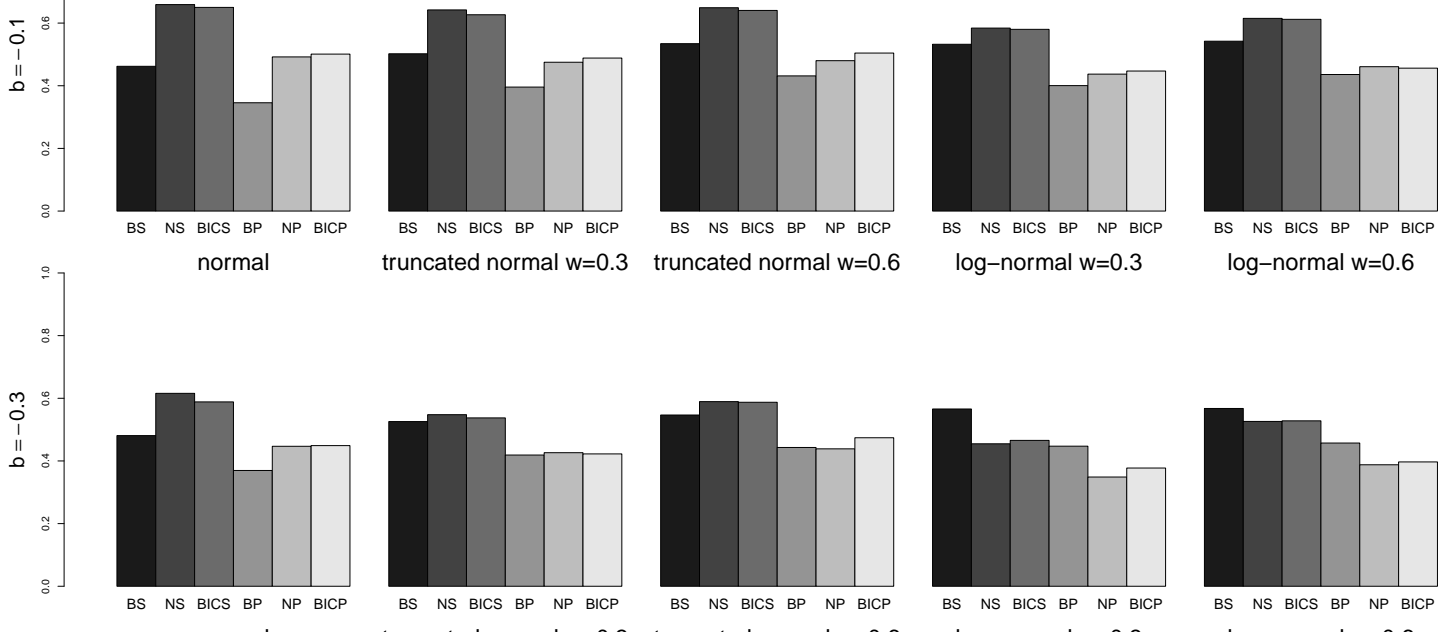

truncated normal $\mathrm{W}=0.3$ truncated normal $\mathrm{w}=0.6$

log-normal w=0.3

log-normal $\mathrm{w}=0.6$

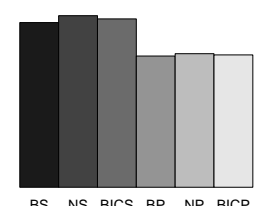

BS NS BICS BP NP BICP truncated normal $\mathrm{w}=0.3$

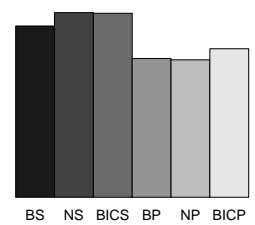

truncated normal $w=0.6$

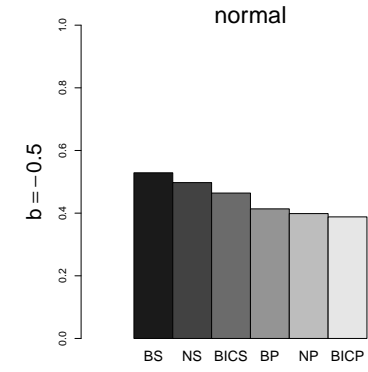

normal

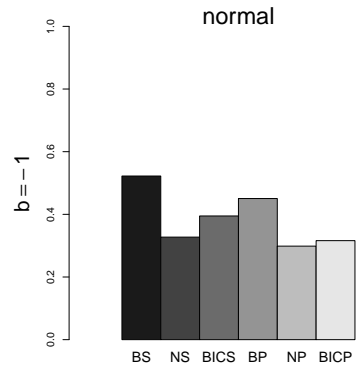

normal
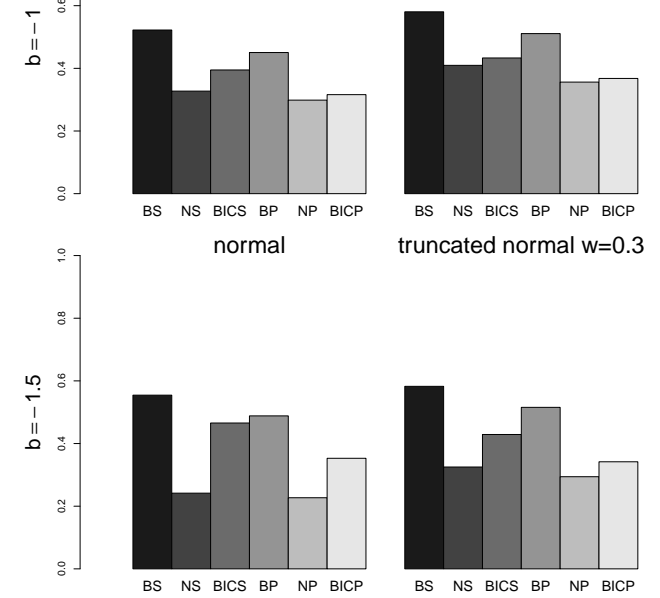

truncated normal $w=0.3$

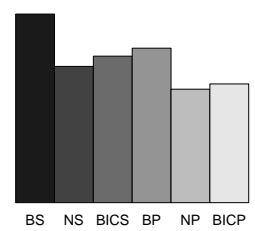

truncated normal $\mathrm{w}=0.6$

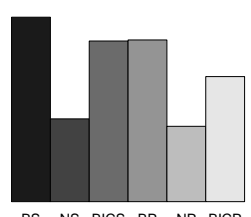

log-normal $w=0.3$
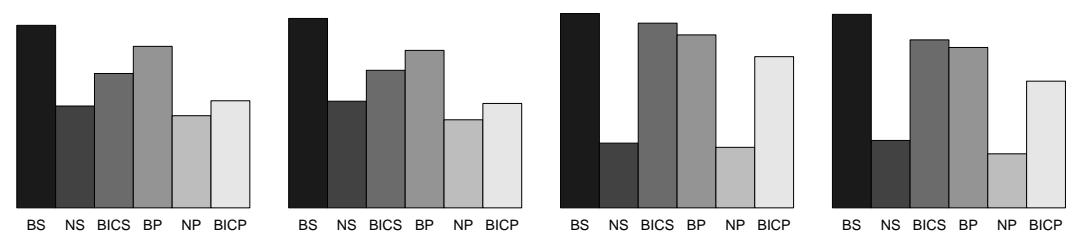

Figure A.12: The probability of Correct MTD Recommendation. The true probablity of toxicity follows a logit model. $a_{i}$ is in scenario 2. The labels BS NS BICS BP NP and BICP for each bar refer to using biomarker model for simple order , non-biomarker model for simple order , BIC model for simple order, biomarker model for partial order, non-biomarker model for partial order and BIC model for partial order. 

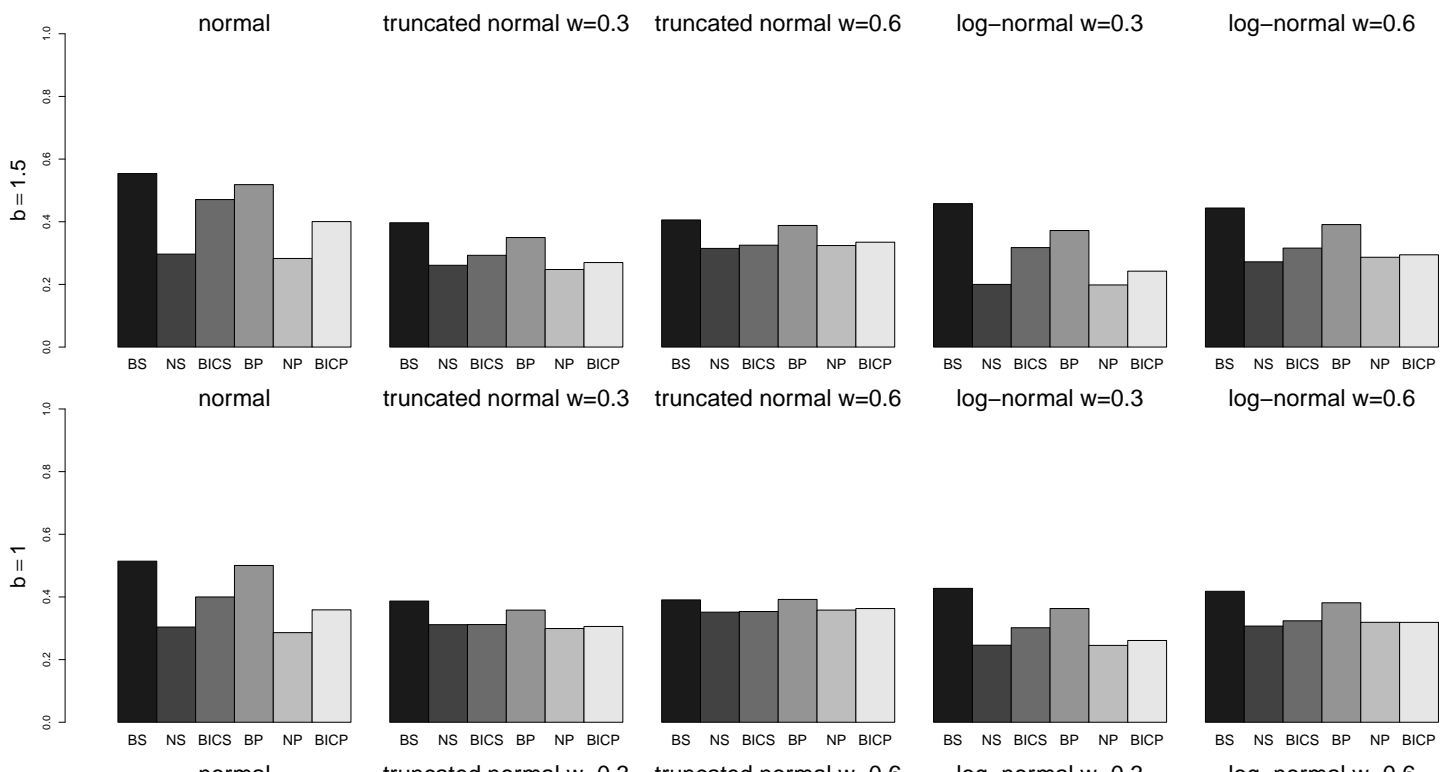

truncated normal $\mathrm{w}=0.3$ truncated normal $\mathrm{w}=0.6$

log-normal w=0.3

log-normal $\mathbf{w}=0.6$
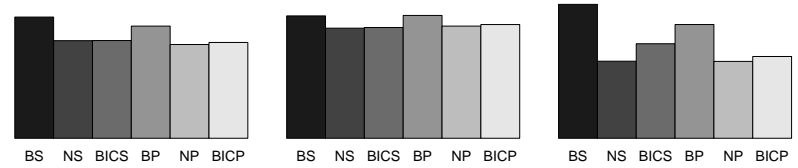

truncated normal $\mathrm{w}=0.3$ truncated normal $\mathrm{w}=0.6$

log-normal $\mathrm{w}=0.3$
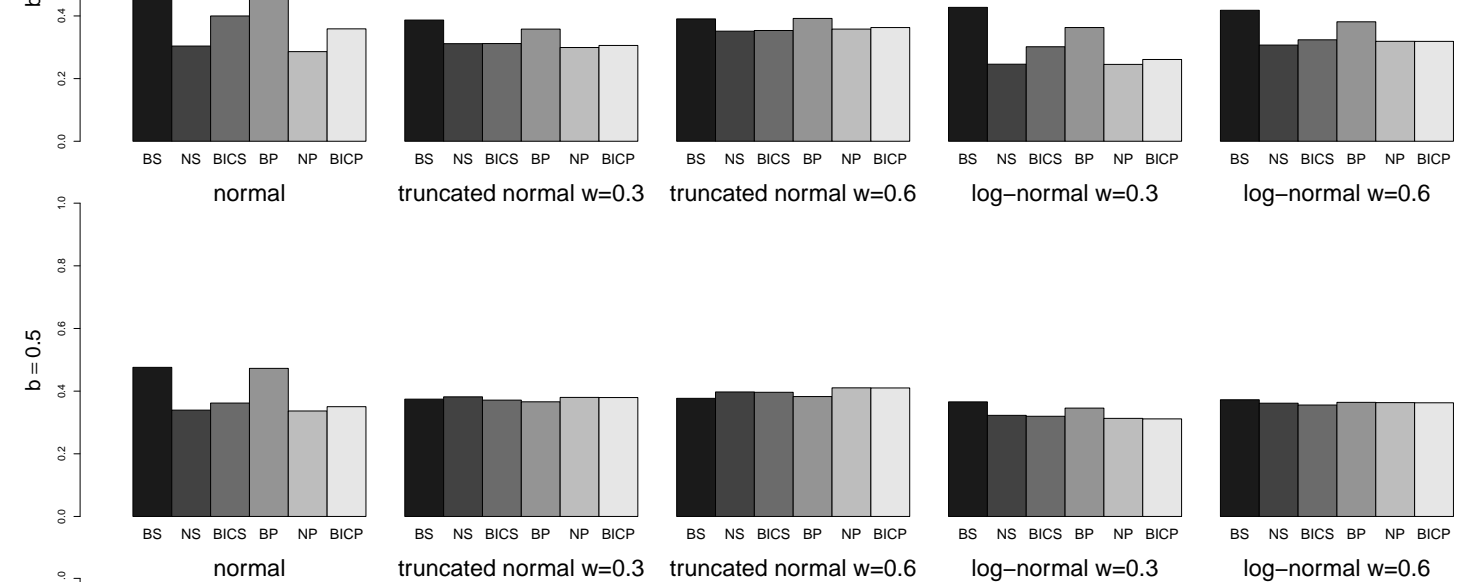

truncated normal $\mathrm{w}=0.3$

truncated normal $\mathrm{W}=0.6$
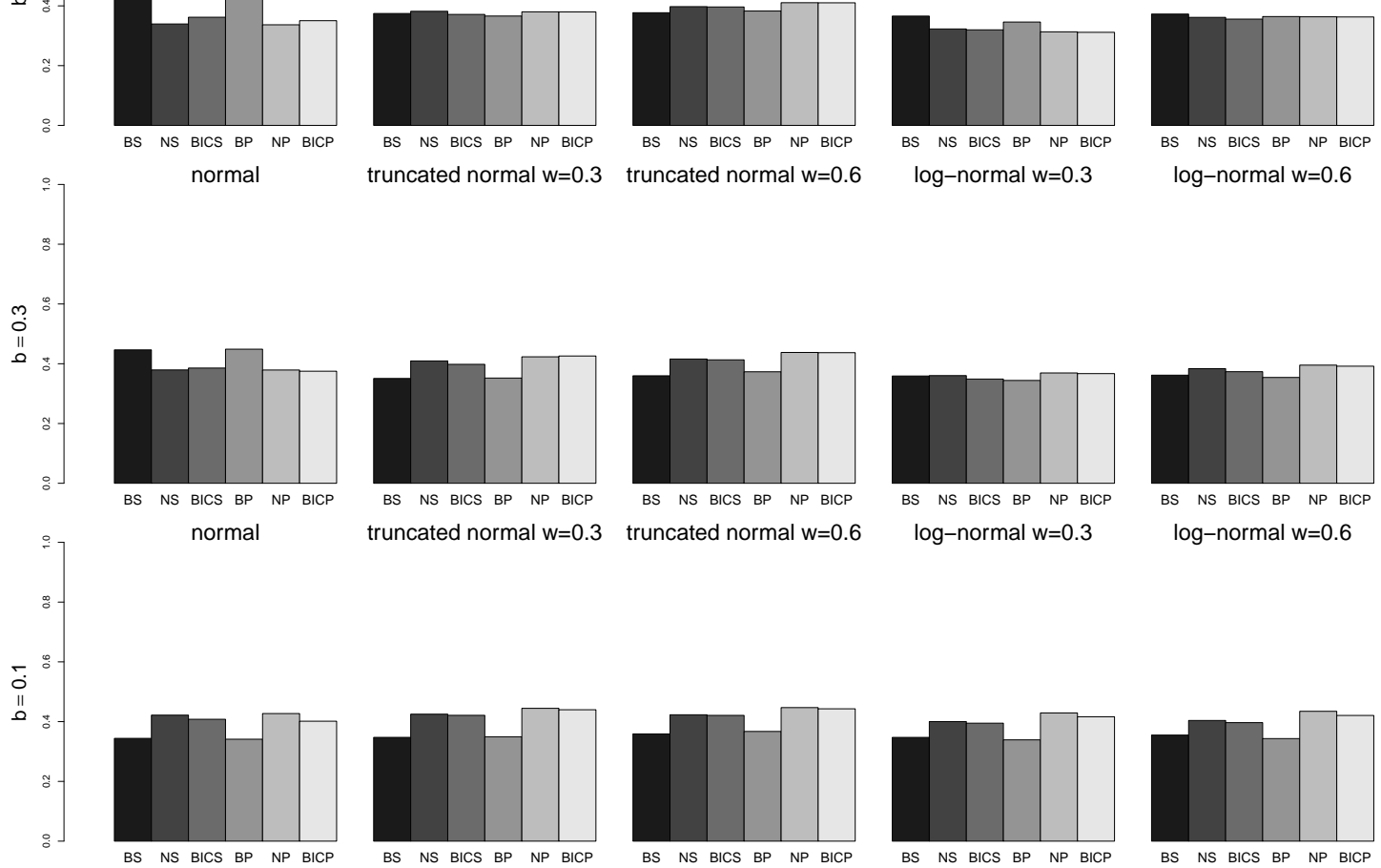

truncated normal $\mathrm{W}=0.3$ truncated normal $\mathrm{W}=0.6$

log-normal $\mathrm{w}=0.3$

log-normal $\mathrm{w}=0.6$
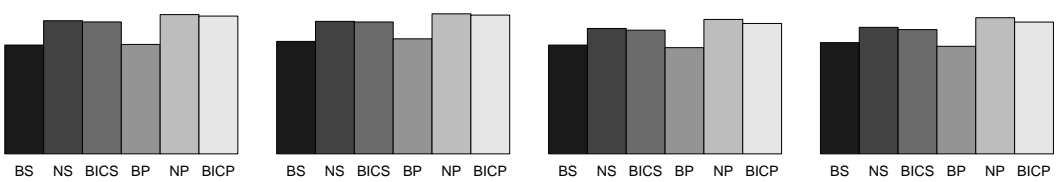

Figure A.13: The percentage of patients treated at the MTDs. The true probablity of toxicity follows a logit model. $a_{i}$ is in scenario 1 . The labels BS NS BICS BP $\mathrm{NP}$ and BICP for each bar refer to using biomarker model for simple order , nonbiomarker model for simple order , BIC model for simple order, biomarker model for partial order, non-biomarker model for partial order and BIC model for partial order. 


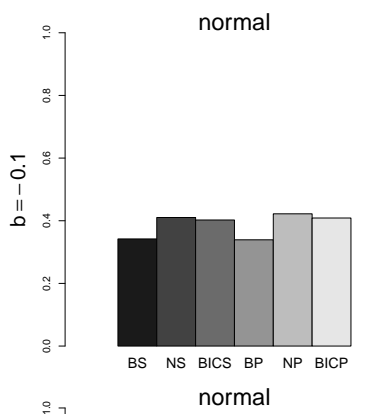

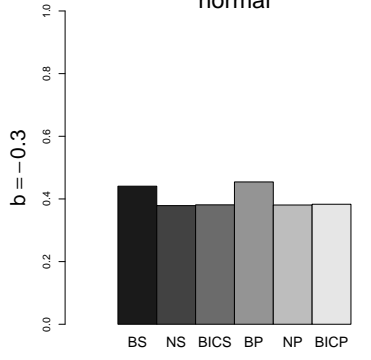
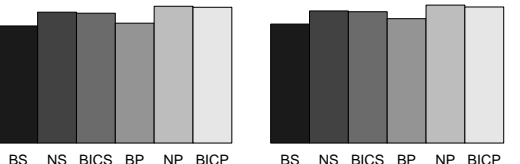

truncated normal $\mathrm{W}=0.3$ truncated normal $\mathrm{W}=0.6$

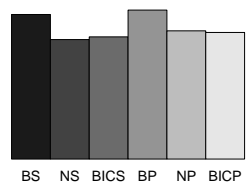

log-normal $\mathrm{W}=0.3$
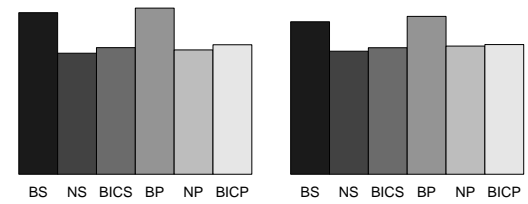

truncated normal $\mathrm{W}=0.3$ truncated normal $\mathrm{w}=0.6$

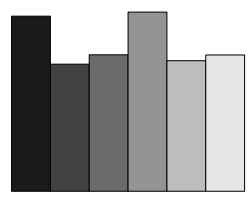

BS NS BICS BP NP BICP

log-normal $\mathrm{w}=0.3$

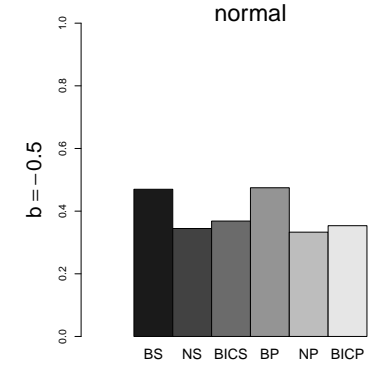

normal
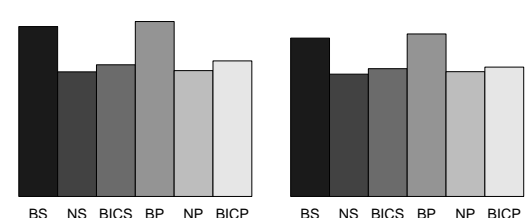

truncated normal $\mathrm{W}=0.3$

truncated normal $\mathrm{W}=0.6$

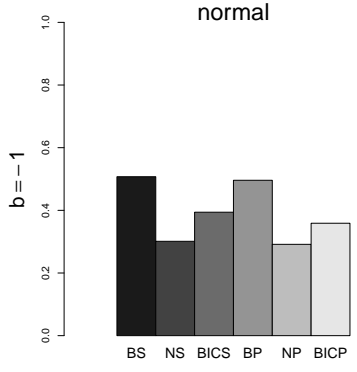

normal
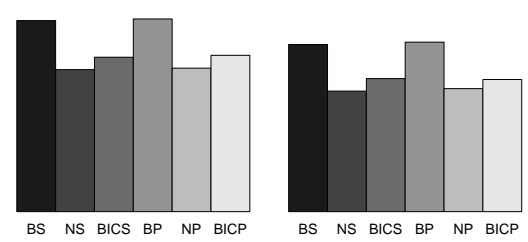

truncated normal $\mathrm{w}=0.3$ truncated normal $\mathrm{w}=0.6$
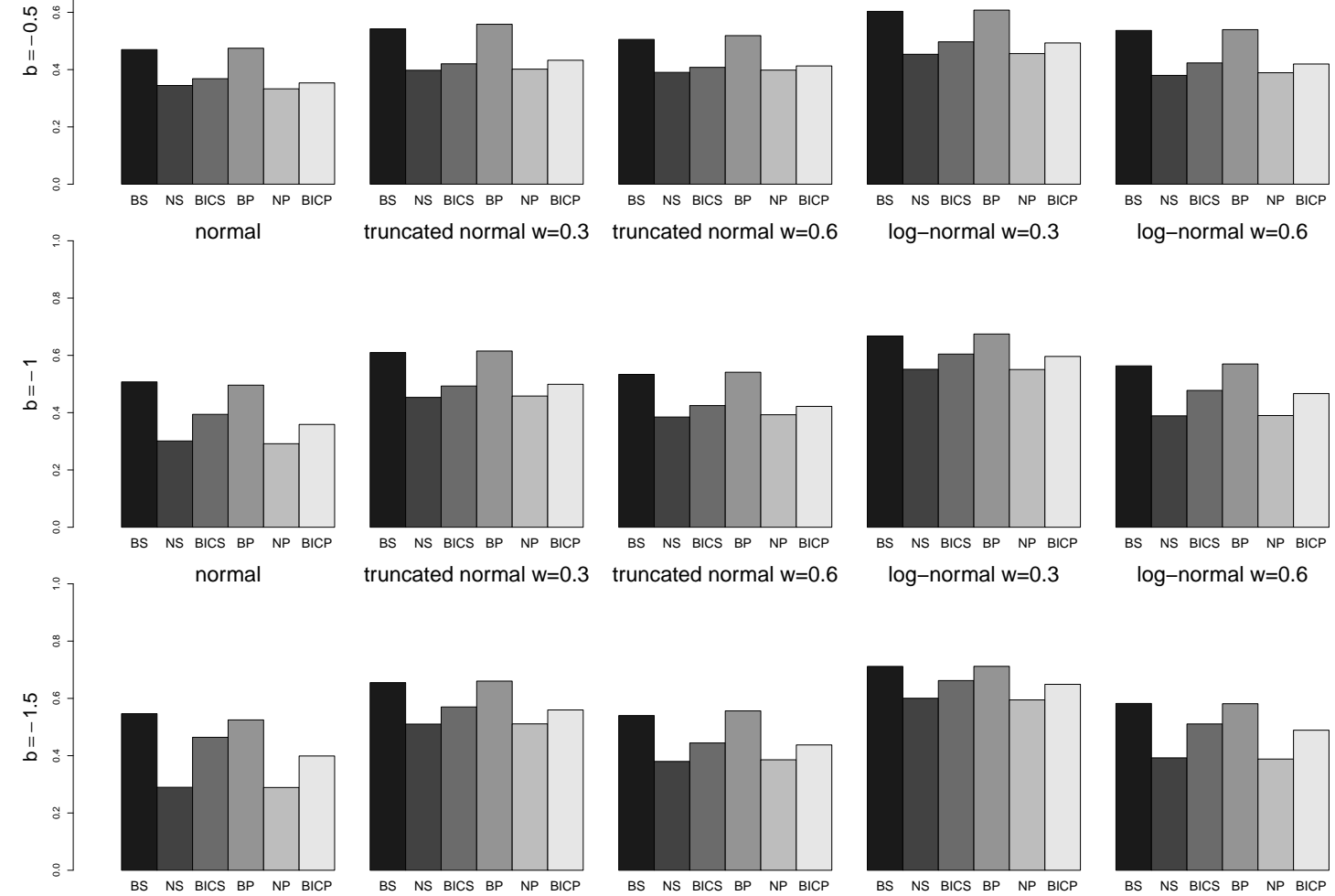

log-normal $\mathrm{w}=0.6$
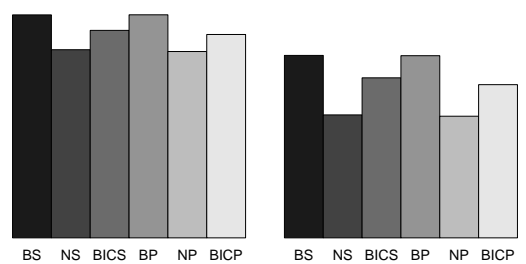

Figure A.14: The percentage of patients treated at the MTDs. The true probablity of toxicity follows a logit model. $a_{i}$ is in scenario 1 . The labels BS NS BICS BP $\mathrm{NP}$ and BICP for each bar refer to using biomarker model for simple order , nonbiomarker model for simple order , BIC model for simple order, biomarker model for partial order, non-biomarker model for partial order and BIC model for partial order. 

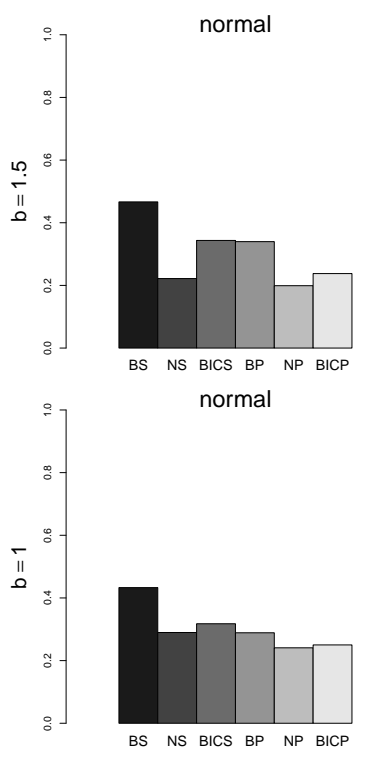

BS NS BICS BP NP BICP
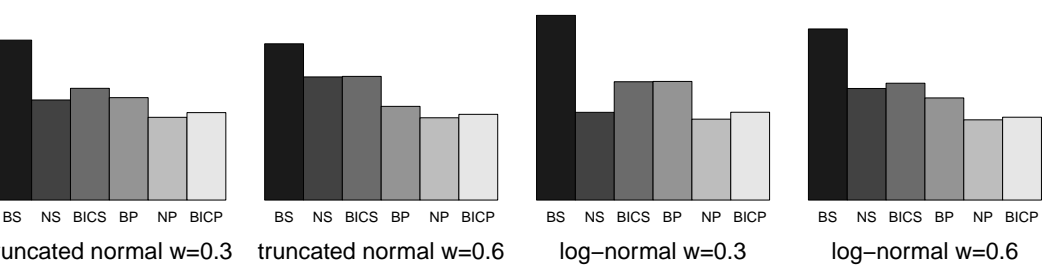

log-normal $\mathrm{W}=0.3$

log-normal $\mathrm{w}=0.6$
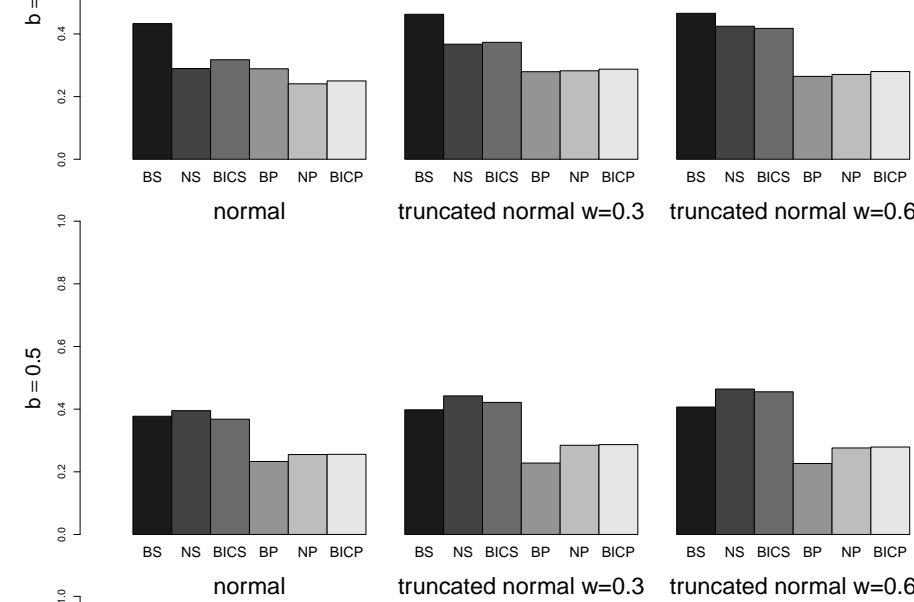

truncated normal $\mathrm{w}=0.3$ truncated normal $\mathrm{w}=0.6$
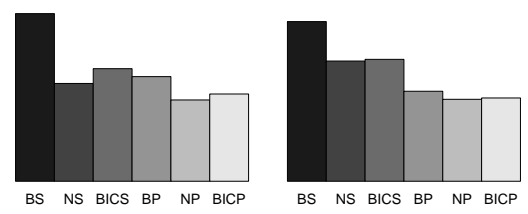

log-normal $\mathrm{w}=0.3$

log-normal $w=0.6$
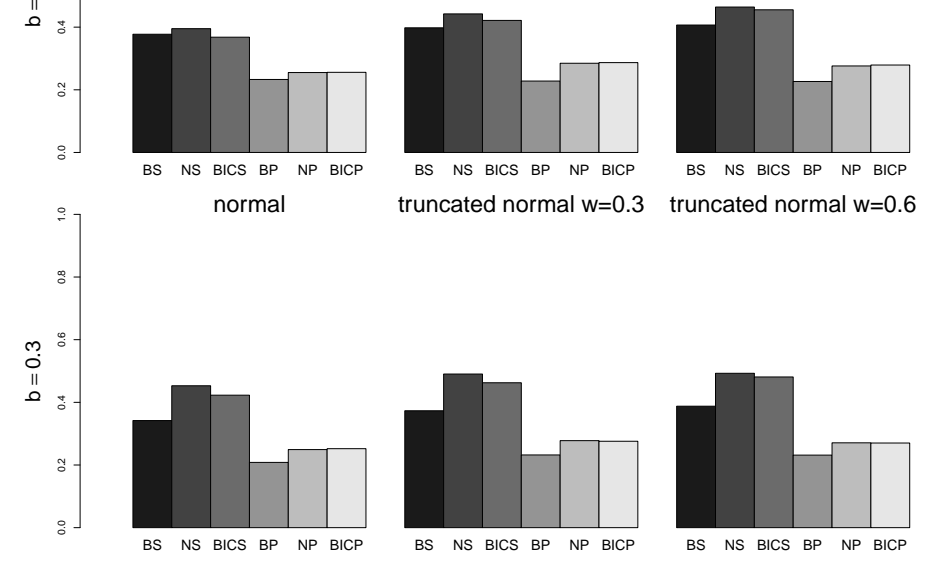

truncated normal $\mathrm{W}=0.3$

BS NS BICS BP NP BICP
truncated normal $\mathrm{W}=0.6$
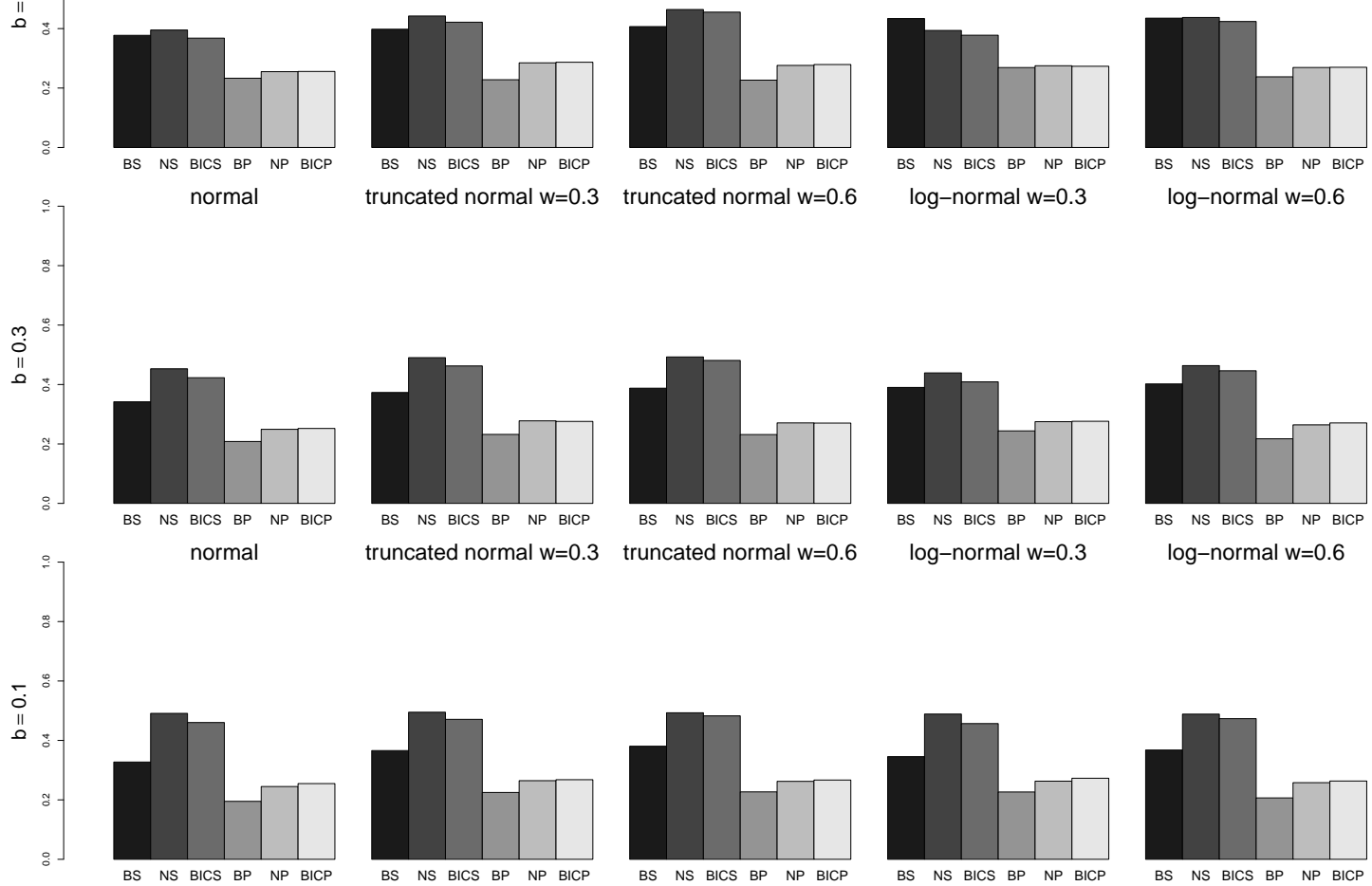

log-normal w=0.3

log-normal $\mathrm{w}=0.6$
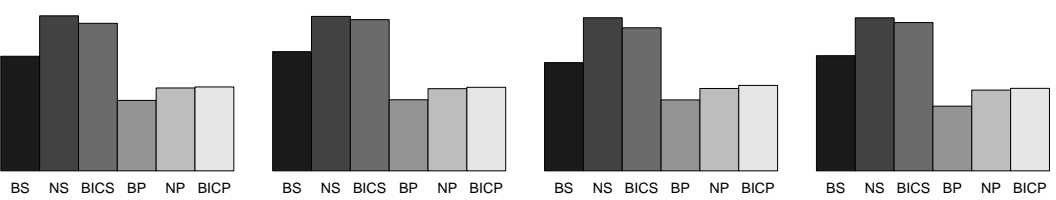

Figure A.15: The percentage of patients treated at the MTDs. The true probablity of toxicity follows a logit model. $a_{i}$ is in scenario 2. The labels BS NS BICS BP $\mathrm{NP}$ and BICP for each bar refer to using biomarker model for simple order , nonbiomarker model for simple order , BIC model for simple order, biomarker model for partial order, non-biomarker model for partial order and BIC model for partial order. 


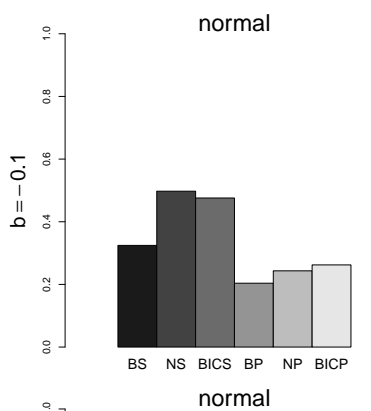

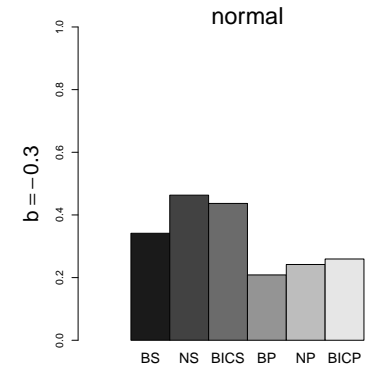

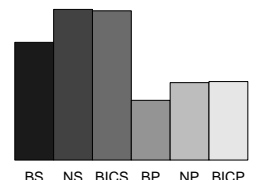

truncated normal $\mathrm{W}=0.3$

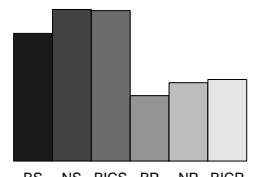

BS NS BICS BP NP BICP truncated normal $\mathrm{w}=0.6$

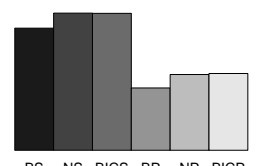

BS NS BICS BP NP BICP

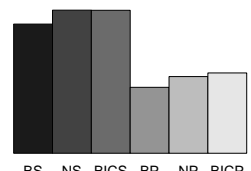

BS NS BICS BP NP BICP
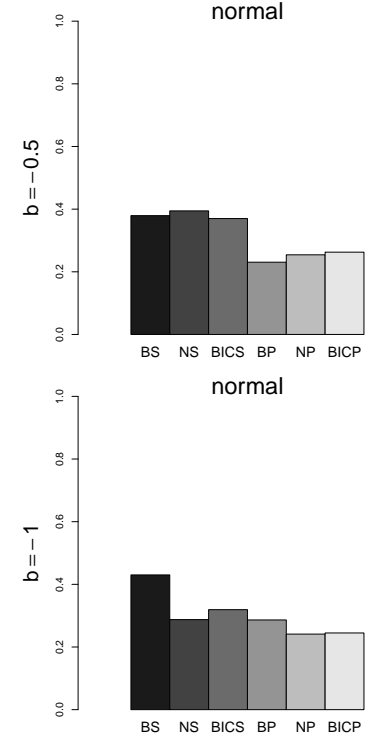

normal
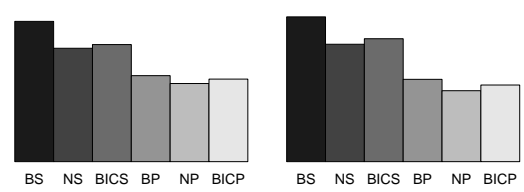

truncated normal $\mathrm{w}=0.3$ truncated normal $\mathrm{w}=0.6$
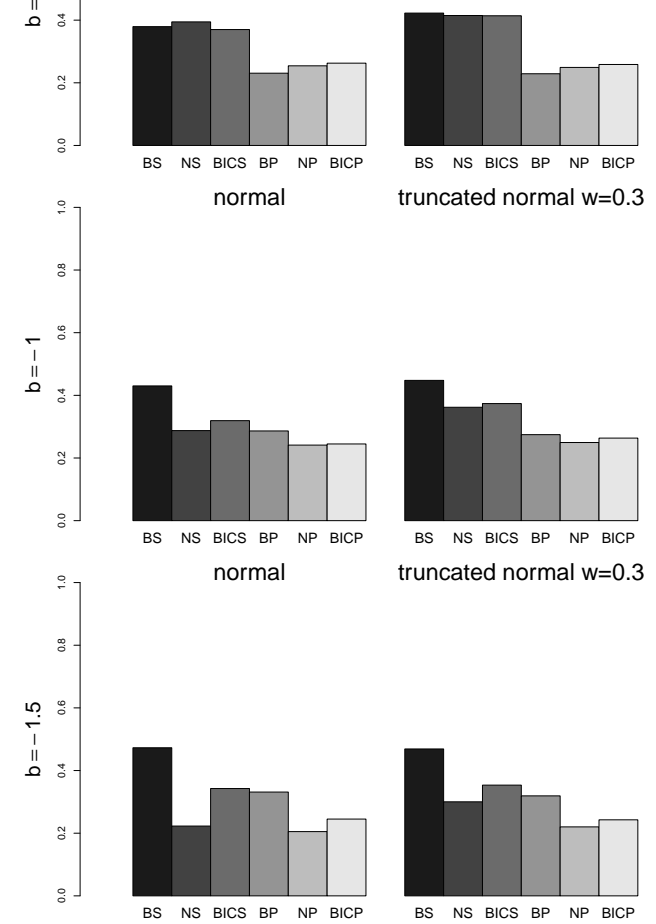

truncated normal $\mathrm{w}=0.3$

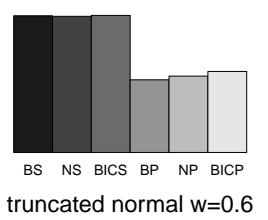

truncated normal $\mathrm{w}=0.6$
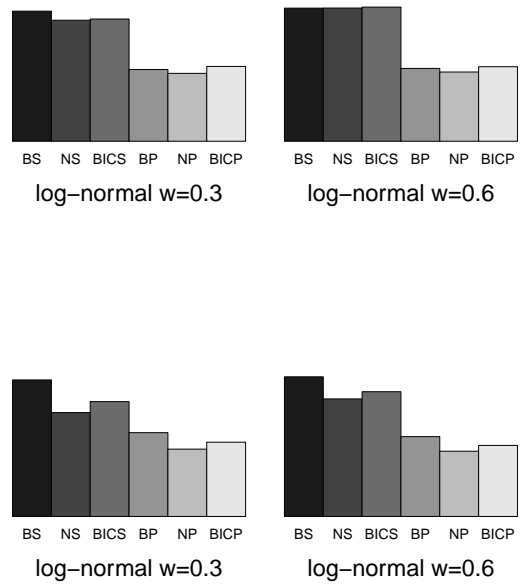

log-normal $\mathrm{w}=0.3$

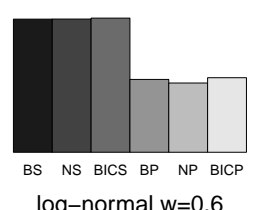

log-normal $\mathrm{w}=0.6$

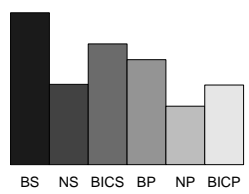

log-normal $\mathrm{w}=0.3$
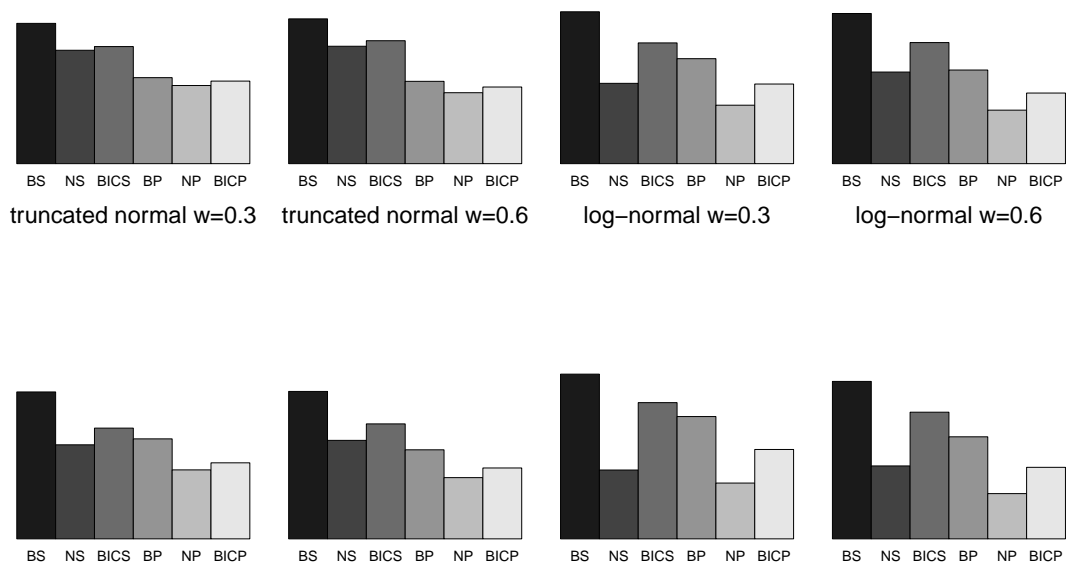

Figure A.16: The percentage of patients treated at the MTDs. The true probablity of toxicity follows a logit model. $a_{i}$ is in scenario 2. The labels BS NS BICS BP $\mathrm{NP}$ and BICP for each bar refer to using biomarker model for simple order , nonbiomarker model for simple order , BIC model for simple order, biomarker model for partial order, non-biomarker model for partial order and BIC model for partial order. 
Table A.1: The proportion of selecting biomarker model as final working model after predetermined sample size of patients. The true probability of toxicity follows an empiric model. The labels S and P refer to BIC method for simple order and BIC method for partial order.

\begin{tabular}{|c|c|c|c|c|c|c|c|c|c|c|}
\hline & \multicolumn{2}{|c|}{ normal } & \multicolumn{2}{|c|}{$\operatorname{trun} \mathrm{w}=0.3$} & \multicolumn{2}{|c|}{$\operatorname{trun} \mathrm{w}=0.6$} & \multicolumn{2}{|c|}{$\log w=0.3$} & \multicolumn{2}{|c|}{$\log \mathrm{w}=0.6$} \\
\hline$b$ & $\mathrm{~S}$ & $\mathrm{P}$ & $\mathrm{S}$ & $\mathrm{P}$ & $\mathrm{S}$ & $\mathrm{P}$ & $\mathrm{S}$ & $\mathrm{P}$ & $\mathrm{S}$ & $\mathrm{P}$ \\
\hline \multicolumn{11}{|c|}{$a=-0.5$} \\
\hline 1.5 & $99 \%$ & $97 \%$ & $66 \%$ & $39 \%$ & $39 \%$ & $16 \%$ & $88 \%$ & $74 \%$ & $59 \%$ & $34 \%$ \\
\hline 1.0 & $90 \%$ & $83 \%$ & $43 \%$ & $20 \%$ & $26 \%$ & $8 \%$ & $74 \%$ & $57 \%$ & $49 \%$ & $26 \%$ \\
\hline 0.5 & $48 \%$ & $41 \%$ & $18 \%$ & $5 \%$ & $12 \%$ & $2 \%$ & $39 \%$ & $26 \%$ & $24 \%$ & $13 \%$ \\
\hline 0.3 & $23 \%$ & $19 \%$ & $8 \%$ & $3 \%$ & $7 \%$ & $1 \%$ & $22 \%$ & $14 \%$ & $15 \%$ & $7 \%$ \\
\hline 0.1 & $8 \%$ & $6 \%$ & $5 \%$ & $1 \%$ & $4 \%$ & $0 \%$ & $7 \%$ & $4 \%$ & $6 \%$ & $2 \%$ \\
\hline-0.1 & $7 \%$ & $6 \%$ & $6 \%$ & $6 \%$ & $8 \%$ & $4 \%$ & $16 \%$ & $13 \%$ & $13 \%$ & $10 \%$ \\
\hline-0.3 & $25 \%$ & $20 \%$ & $17 \%$ & $14 \%$ & $15 \%$ & $8 \%$ & $46 \%$ & $43 \%$ & $37 \%$ & $31 \%$ \\
\hline-0.5 & $46 \%$ & $42 \%$ & $31 \%$ & $27 \%$ & $25 \%$ & $16 \%$ & $68 \%$ & $66 \%$ & $57 \%$ & $50 \%$ \\
\hline-1.0 & $89 \%$ & $84 \%$ & $64 \%$ & $62 \%$ & $56 \%$ & $45 \%$ & $93 \%$ & $91 \%$ & $87 \%$ & $80 \%$ \\
\hline .5 & $99 \%$ & $96 \%$ & $85 \%$ & $80 \%$ & $78 \%$ & $63 \%$ & $98 \%$ & $98 \%$ & $95 \%$ & $90 \%$ \\
\hline \multicolumn{11}{|c|}{$a=0.42$} \\
\hline 1.5 & $98 \%$ & $96 \%$ & $\%$ & $31 \%$ & $27 \%$ & $10 \%$ & $80 \%$ & $55 \%$ & $45 \%$ & $23 \%$ \\
\hline 1.0 & $85 \%$ & $76 \%$ & $40 \%$ & $22 \%$ & $18 \%$ & $7 \%$ & $65 \%$ & $46 \%$ & $34 \%$ & $18 \%$ \\
\hline 0.5 & $44 \%$ & $32 \%$ & $16 \%$ & $8 \%$ & $9 \%$ & $3 \%$ & $34 \%$ & $26 \%$ & $18 \%$ & $10 \%$ \\
\hline 0.3 & $20 \%$ & $14 \%$ & $10 \%$ & $4 \%$ & $5 \%$ & $2 \%$ & $18 \%$ & $14 \%$ & $11 \%$ & $7 \%$ \\
\hline 0.1 & $7 \%$ & $4 \%$ & $4 \%$ & $2 \%$ & $2 \%$ & $0 \%$ & $7 \%$ & $5 \%$ & $6 \%$ & $3 \%$ \\
\hline-0.1 & $6 \%$ & $5 \%$ & $6 \%$ & $1 \%$ & $4 \%$ & $1 \%$ & $15 \%$ & $7 \%$ & $10 \%$ & $7 \%$ \\
\hline-0.3 & $18 \%$ & $14 \%$ & $13 \%$ & $5 \%$ & $11 \%$ & $5 \%$ & $43 \%$ & $33 \%$ & $33 \%$ & $24 \%$ \\
\hline-0.5 & $38 \%$ & $31 \%$ & $25 \%$ & $13 \%$ & $22 \%$ & $10 \%$ & $72 \%$ & $59 \%$ & $56 \%$ & $44 \%$ \\
\hline-1.0 & $86 \%$ & $78 \%$ & $61 \%$ & $49 \%$ & $53 \%$ & $37 \%$ & $96 \%$ & $90 \%$ & $87 \%$ & $78 \%$ \\
\hline-1.5 & $97 \%$ & $94 \%$ & $88 \%$ & $81 \%$ & $77 \%$ & $63 \%$ & $99 \%$ & $98 \%$ & $96 \%$ & $92 \%$ \\
\hline
\end{tabular}


Table A.2: The proportion of selecting biomarker model as final working model after predetermined sample size of patients. The true probability of toxicity follows a logit model. The labels $\mathrm{S}$ and $\mathrm{P}$ refer to BIC method for simple order and BIC method for partial order.

\begin{tabular}{|c|c|c|c|c|c|c|c|c|c|c|}
\hline \multirow[b]{2}{*}{$b$} & \multicolumn{2}{|c|}{ normal } & \multicolumn{2}{|c|}{$\operatorname{trun} \mathrm{w}=0.3$} & \multicolumn{2}{|c|}{$\operatorname{trun} \mathrm{w}=0.6$} & \multicolumn{2}{|c|}{$\log w=0.3$} & \multicolumn{2}{|c|}{$\log \mathrm{w}=0.6$} \\
\hline & $\mathrm{S}$ & $\mathrm{P}$ & $\mathrm{S}$ & $\mathrm{P}$ & $\mathrm{S}$ & $\mathrm{P}$ & $\mathrm{S}$ & $\mathrm{P}$ & $\mathrm{S}$ & $\mathrm{P}$ \\
\hline \multicolumn{11}{|c|}{$a=-0.5$} \\
\hline 1.5 & $85 \%$ & $78 \%$ & $38 \%$ & $17 \%$ & $23 \%$ & $7 \%$ & $67 \%$ & $52 \%$ & $43 \%$ & $24 \%$ \\
\hline 1.0 & $59 \%$ & $49 \%$ & $23 \%$ & $8 \%$ & $15 \%$ & $3 \%$ & $46 \%$ & $35 \%$ & $29 \%$ & $17 \%$ \\
\hline 0.5 & $22 \%$ & $18 \%$ & $9 \%$ & $3 \%$ & $7 \%$ & $1 \%$ & $24 \%$ & $14 \%$ & $16 \%$ & $8 \%$ \\
\hline 0.3 & $13 \%$ & $10 \%$ & $6 \%$ & $2 \%$ & $4 \%$ & $1 \%$ & $13 \%$ & $6 \%$ & $10 \%$ & $3 \%$ \\
\hline 0.1 & $6 \%$ & $5 \%$ & $4 \%$ & $1 \%$ & $3 \%$ & $0 \%$ & $5 \%$ & $3 \%$ & $4 \%$ & $2 \%$ \\
\hline-0.1 & $5 \%$ & $5 \%$ & $5 \%$ & $4 \%$ & $6 \%$ & $4 \%$ & $10 \%$ & $8 \%$ & $8 \%$ & $6 \%$ \\
\hline-0.3 & $12 \%$ & $10 \%$ & $8 \%$ & $8 \%$ & $10 \%$ & $5 \%$ & $26 \%$ & $25 \%$ & $21 \%$ & $18 \%$ \\
\hline-0.5 & $22 \%$ & $19 \%$ & $15 \%$ & $13 \%$ & $14 \%$ & $8 \%$ & $49 \%$ & $45 \%$ & $38 \%$ & $32 \%$ \\
\hline-1.0 & $58 \%$ & $52 \%$ & $37 \%$ & $35 \%$ & $30 \%$ & $21 \%$ & $82 \%$ & $80 \%$ & $70 \%$ & $62 \%$ \\
\hline-1.5 & $84 \%$ & $79 \%$ & $62 \%$ & $60 \%$ & $52 \%$ & $40 \%$ & $96 \%$ & $92 \%$ & $86 \%$ & $81 \%$ \\
\hline \multicolumn{11}{|c|}{$a=0.42$} \\
\hline 1.5 & $71 \%$ & $56 \%$ & $31 \%$ & $16 \%$ & $15 \%$ & $5 \%$ & $52 \%$ & $37 \%$ & $29 \%$ & $15 \%$ \\
\hline 1.0 & $43 \%$ & $33 \%$ & $18 \%$ & $9 \%$ & $10 \%$ & $3 \%$ & $38 \%$ & $27 \%$ & $21 \%$ & $11 \%$ \\
\hline 0.5 & $16 \%$ & $12 \%$ & $8 \%$ & $4 \%$ & $5 \%$ & $1 \%$ & $18 \%$ & $14 \%$ & $11 \%$ & $6 \%$ \\
\hline 0.3 & $10 \%$ & $6 \%$ & $6 \%$ & $2 \%$ & $3 \%$ & $1 \%$ & $11 \%$ & $8 \%$ & $8 \%$ & $4 \%$ \\
\hline 0.1 & $5 \%$ & $3 \%$ & $3 \%$ & $2 \%$ & $2 \%$ & $0 \%$ & $5 \%$ & $4 \%$ & $4 \%$ & $2 \%$ \\
\hline-0.1 & $4 \%$ & $3 \%$ & $5 \%$ & $1 \%$ & $3 \%$ & $1 \%$ & $8 \%$ & $4 \%$ & $5 \%$ & $4 \%$ \\
\hline-0.3 & $8 \%$ & $6 \%$ & $6 \%$ & $1 \%$ & $6 \%$ & $2 \%$ & $19 \%$ & $11 \%$ & $14 \%$ & $11 \%$ \\
\hline-0.5 & $14 \%$ & $12 \%$ & $10 \%$ & $3 \%$ & $9 \%$ & $3 \%$ & $36 \%$ & $24 \%$ & $27 \%$ & $20 \%$ \\
\hline-1.0 & $41 \%$ & $35 \%$ & $22 \%$ & $12 \%$ & $20 \%$ & $9 \%$ & $73 \%$ & $58 \%$ & $57 \%$ & $44 \%$ \\
\hline-1.5 & $69 \%$ & $58 \%$ & $40 \%$ & $26 \%$ & $37 \%$ & $19 \%$ & $90 \%$ & $81 \%$ & $77 \%$ & $65 \%$ \\
\hline
\end{tabular}




\section{Bibliography}

[1] Akaba, K., Kimura, T., Sasaki, A., Tanabe, S., Ikegami, T., Hashimoto, M., Umeda, H., Yoshida, H., Umetsu, K., Chiba, H., Yuasa, I., Hayasaka, K. (1998), Neonatal hyperbilirubinemia and mutation of the bilirubin uridine diphosphateglucuronosyltransferase gene: a common missense mutation among Japanese, Koreans and Chinese. Biochem Mol Biol Int 46, 21-26.

[2] Azriel, D. (2005), A note on the robustness of the continual reassessment method. Statistics and Probability Letters 82(5), 902-906.

[3] Babb, J., Rogatko, A., Zacks, S. (1998), Cancer phase I clinical trials: efficient dose escalation with overdose control. Statistics in Medicine 17(10), 1103-1120.

[4] Berenson, JR., Yellin, O., Patel, R., Duvivier, H., Nassir, Y., Mapes, R., Abaya, CD., Swift, RA. (2009), A phase I study of samarium Lexidronam / bortezomib combination therapy for the treatment of relapsed or regractory multiple myeloma. Clinical Cancer Research 15(3), 1069-1075.

[5] Cheung, YK., Chappell R. (2002), A simple technique to evaluate model sensitivity in the continual reassessment method. Biometrics 58(3), 671-674.

[6] Conaway, MR., Dunbar, S., Peddada, SD. (2004), Designs for single- or multiple-agent phase I trials. Biometrics 60(3), 661-669. 
[7] Durham, SD., Flournoy, N., and Rosenberger, WF. (1997), A random walk rule for phase I clinical trials. Biomatrics 53(2), 745-760.

[8] Falandry, C., Horard, B., Alexandre, J., Deplanque, G., Cojocarasu, O., Salvat, J., et al. (2012), Correlation of short telomeres (ST) with vulnerability, toxicity, and early death in elderly AOC patients receiving carboplatin: A multicenter GINECO trial. Journal of clinical oncology 30 supple, Abstract No: 9011.

[9] Freidlin, B., McShane, LM., and Korn, EL. (2010), Randomized clinical trials with biomarkers: design issues. J Natl Cancer Inst 102(3), 152-160.

[10] Fujita, K., Ando, Y., Nagashima, F., Yamamoto, W., Eodo, H., Araki, K., Kodama, K., Miya, T., Narabayashi, M., Sasaki, Y. (2007), Genetic linkage of UGT1A7 and UGT1A9 polymorphisms to UGT1A1*6 is associated with reduced activity for SN-38 in Japanese patients with cancer. Cancer Chemother Pharmacol 60, 515-522.

[11] Garg, MB., Lincz, LF., Adler, K., Scorgie, FE., Ackland, SP., Sakoff, JA. (2012), Predicting 5-fluorouracil toxicity in colorectal cancer patients from peripheral blood cell telomere length: a multivariate analysis. British Journal of Cancer 107, 1525-1533.

[12] Hwang, J. and Peddada, S. (1994), Confidence interval estimation subject to order restrictions. Annals of Statistics 22, 67-93.

[13] Ivanova, A., Wang, K. (2006), Bivariate isotonic design for dose-finding with ordered groups. Statistics in Medicine 25(12), 2018-2026.

[14] Karapetis, CS., Khambata-Ford, S., Jonker, DJ., O'Callaghan, CJ., Tu, D., Tebbutt, NC., Simes, RJ., Chalchal, H., Shapiro, JD., Robitaille, S., Price, 
TJ., Shepherd, L., Au, HJ., Langer, C., Moore, MJ., Zalcberg, JR. (2008), K-ras mutations and benefit from cetuximab in advanced colorectal cancer. $N$ Engl J Med 359(17), 1757-1765.

[15] Kim, KP., Kim, HS., Sym, SJ., Bae, KS., Hong, YS., Chang, HM., Lee, JL., Kang, YK., Lee, JS., Shin, JG., Kim, TW. (2013), A UGT1A1*28 and *6 genotype-directed phase I dose-escalation trial of irinotecan with fixeddose capecitabine in Korean patients with metastatic colorectal cancer. Cancer Chemother Pharmacol 71, 1609-1617.

[16] Lee, SM., Cheung, YK. (2009), Model calibration in the continual reassessment method. Clinical Trials 6, 227-238.

[17] Lokich J. (2001), Phase I clinical trial of weekly combined topotecan and irinotecan. American Journal of Clinical Oncology 24(4), 336-340.

[18] Mandrekar, SJ., Grothey, A., Goetz, MP., Sargent, DJ. (2005), Clinial trial designs for prospective validation of biomarkers. Am J Pharmacogenomics 5(5) 317-325.

[19] Morita, S. (2011), Application of the continual reassessment method to a phase I dose-finding trial in Japanese patients: East meets West. Statistics in Medicine 30(17), 2090-2097.

[20] O'Quigley, J. (2005), Theoretical study of the continual reassessment method. Journal of Statistical Planning and Inference 136(6), 1765-1780.

[21] O'Quigley, J., Paoletti, X. (2003), Continual reassessment method for ordered groups. Biometrics 59, 430-440. 
[22] O'Quigley, J., Pepe, M., Fisher, L. (1990), Continual reassessment method: A practical design for Phase I clinical trials in cancer. Biometrics 46, 33-48.

[23] O'Quigley, J., Shen, LZ. (1996) Continual reassessment method: A likelihood approach. Biometrics 52(2), 673-684.

[24] O'Quigley, J., Shen, LZ., Gamst, A. (1999), Two-sample continual reassessment method. Journal of Biopharmaceutical Statistics 9(1), 17-44.

[25] O'Quigley, J., Zohar, S. (2010), Retrospective robustness of the continual reassessment method. Journal of Biopharmaceutical Statistics 5, 1013-1025.

[26] Patnaik, A., Warner, E., Michael, M., Egorin, MJ., Moore, MJ., Siu, LL., Fracasso, PM., Rivkin, S., Kerr, I., Litchman, M., Oza, AM. (2000), Phase I dose-finding and pharmacokinetic study of paclitaxel and carboplatin with oral valspodar in patients with advanced solid tumors. Journal of the National Cancer Institute 18, 3677-3689.

[27] Robertson, T., Wright, F., and Dykstra, R. (1988), Order Restricted Statistical Inference. New York: Wiley.

[28] Schawrz, G. (1978), Estimating the dimension of a model. Annals of Statistics 6, 461-464.

[29] Storer, BE. (1989), Design and analysis of phase I clinical trials. Biometrics 45(3), 925-937.

[30] Shiryayev, AN. (1984), Probability (Trans. R. P. Boas). New York: SpringerVerlag.

[31] Sunakawa, Y., Fujita, K., Ichikawa, W., Ishida, H., Yamashita, K., Araki, K., Miwa, K., Kawara, K., Akiyama, Y., Yamamoto, W., Nagashima, F., Saji, 
S., Sasaki, Y. (2012), A phase I study of infusional 5-fluorouracil, leucovorin, oxaliplatin and irinotecan in Japanese patients with advanced colorectal cancer who harbor UGT1A1*1/*1, * $1 /{ }^{*} 6$ or $* 1 / * 28$. Oncology 82(4), 242-248.

[32] Thall, PF., Nguyen, HQ., Estey, EH. (2008), Patient-specific dose finding based on bivariate outcomes and covariates. Biometrics 64(4), 1126-1136.

[33] Wages, NA. (2010), Dose-finding designs for muti-drug combinations. Unpublished Ph.D. Thesis.

[34] Wages, NA., Conaway, MR., O'Quigley, J. (2011), Continual reassessment method for partial ordering. Biometrics 67(4), 1-22.

[35] Wages, NA., Conaway, MR., O'Quigley, J. (2011), Dose-finding design for multi-drug combinations. Clinical Trials 8(4), 380-389.

[36] Yuan, Z., Chapell, R. (2004), Isotonic designs for phase I cancer clinical trials with multiple risk groups. Clinical Trials 1(6), 499-508. 